\title{
Identifying Human Host Cell Protein Targets of the Bartonella Effector Protein (Bep) Fic Domains
}

\author{
Vaughan Trounson
}

A thesis submitted to Victoria University of Wellington in fulfilment of the requirements for the degree of Masters of Biomedical Science

Victoria University of Wellington

2016 



\section{Abstract}

The genus Bartonellae represents an increasing number of emerging bacterial pathogens that utilises an unusual infection strategy, parasitising the red blood cells of their mammalian host. The most common species to infect humans are $B$. henselae and $B$. quintana. $B$. henselae is transmitted between cats by the cat flea, although occasionally infects humans via cat scratches or bites, causing cat-scratch disease (CSD). CSD is characterised by enlarged tender lymph nodes and fever. $B$. henselae also infects the endothelial cells of both its hosts; likely a factor in disease progression. B. quintana, the cause of trench fever during WWI, is spread between people by the body louse. Trench fever is characterised by relapsing fever, headache, and bone pain. B. quintana is also able to infect human endothelial cells. These bacteria secrete a range of Bartonella effector proteins (Beps) via a Type IV secretion system, directly into endothelial cells, subverting host cell processes and resulting in internalisation of the bacteria.

Beps have a range of functions, many of which are not fully characterised. $B$. henselae secretes three Beps (BepA-C) that contain a filamentation induced by $\underline{\mathrm{c}} \mathrm{AMP}$ (Fic) domain and a Bartonella Intracellular Delivery (BID) domain, with BepA being the best studied. BepA's BID domain is responsible for intracellular delivery as well as inhibition of apoptosis by the host cell, however the exact function of the Fic domain remains unknown. Fic-containing bacterial toxins catalyse the transfer of an AMP moiety from ATP onto a host cell protein. This AMPylation frequently inactivates these proteins resulting in disrupted host cell processes and cytotoxicity. BepA has previously been shown to possess AMPylation activity, although the host target protein(s) are unknown. Evidence suggests that these proteins are novel targets.

The aim of this study was to create protein constructs containing these Fic domains, and to develop techniques to identify the host cell target proteins post AMPylation. To this end, both a fluorescent ATP analogue and a fluorescent click chemistry based approach were utilised. While no target protein was identified, a basic methodology was developed for protein production and target protein identification that could be further developed. 


\section{Acknowledgements}

Firstly, I would like to thank my supervisor, Dr Joanna MacKichan for giving me the opportunity to work on this project. Thank you for all your support throughout my Masters, in particular for always being available to provide help and feedback, even as late as midnight. It was your lectures on Microbiology in BMSC 301 that really helped me settle on an area of molecular biology I wanted to pursue further. Thank you for all your help with setting up funding so that I'm able to attend the conference in Montana. I couldn't have picked a better supervisor.

I would also like to thank Raphael, Alistair, and Varun for helping me get to grips with my initial protein experiments, as well as protein work in general. To the Ackerley lab, you guys were always so helpful in answering my various questions, as well as always providing friendly faces in the lab to talk to while I was waiting for an experiment to complete. Thanks also to Dr Lifeng Peng, Sven, and Richard from AM for all your help running samples on the Mass Spec, as well as a thank you to Bhumi for your help in teaching me how to do western blots properly. I'd also like to thank Dr John Miller and Dr Jane Koehler for supplying the HeLa cells and B. quintana gDNA, respectively. I'd also like to thank the American Society for Microbiology for permission to reproduce the Bartonella Lifecycle diagram illustrated in Figure 1.1.

To all the staff and students of SBS, in particular my office mates: James, Jasmine, Jacob, Jen, Jack, Kate, Sonja, Melz and Rory. You guys made doing a Masters less stressful than it otherwise could've been. In particular, a special mention to my lab mate Jacob, for always telling me on a rough day "you got this". I'd also like to thank my flatmate Colin for all your help reading my final draft. Finally, a huge thank you to all my friends and family, in particular Mum, Dad, and Jess for accommodating my busy schedule during both my undergraduate and postgraduate years at university. I couldn't have done it without all your support.

Finally, thank you to both the Victoria University of Wellington Science Faculty, and the American Society of Rickettsiology for providing the funding that enabled me to attend the conference in Montana. 


\section{Table of Contents}

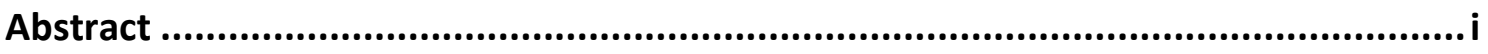

Acknowledgements ................................................................................................ ii

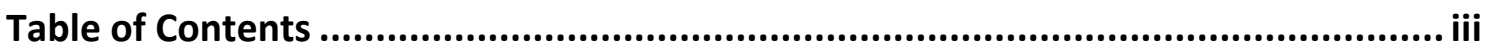

List of Tables and Figures ....................................................................................... viii

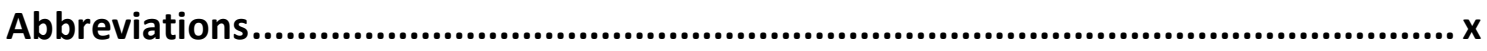

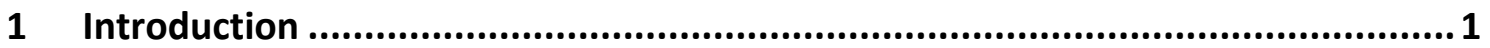

1.1 Bartonella Species and Associated Disease ........................................................... 1

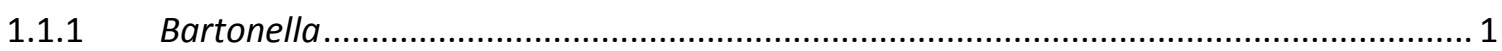

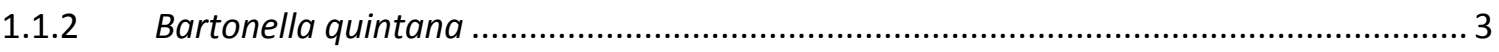

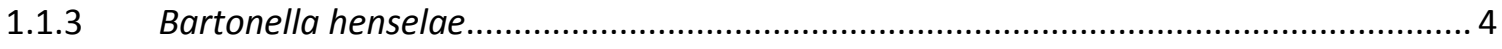

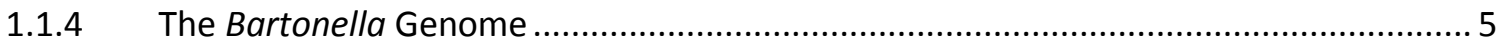

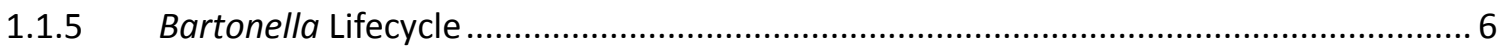

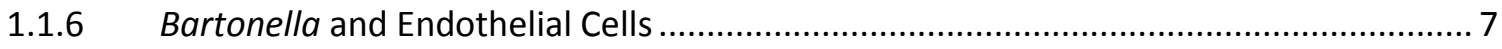

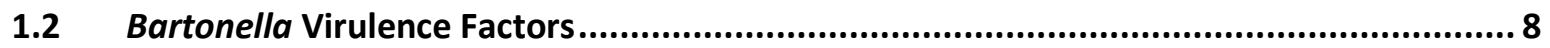

1.2.1 VirB/D4 T4SS and Secreted Effector Proteins ................................................................. 9

1.3 Bartonella VirB/D4 Secreted Effector Proteins .............................................................. 10

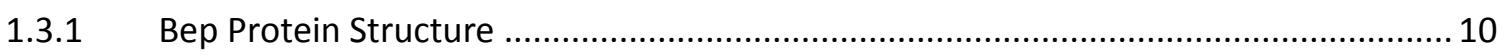

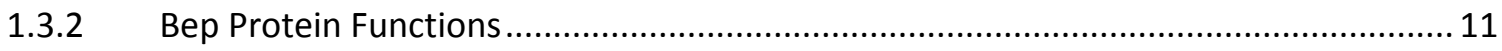

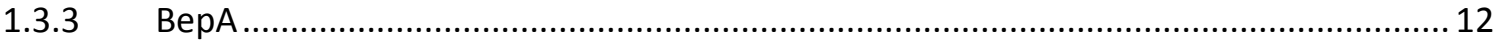

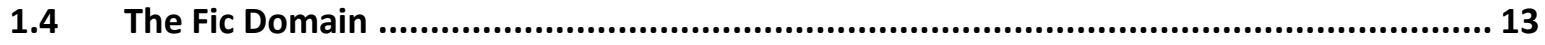

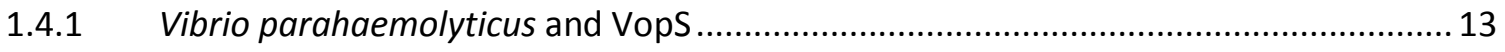

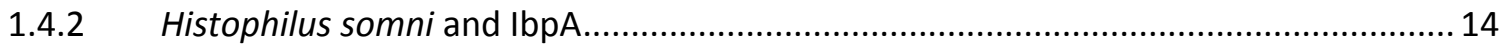

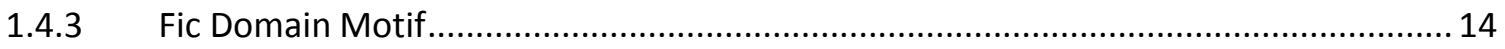

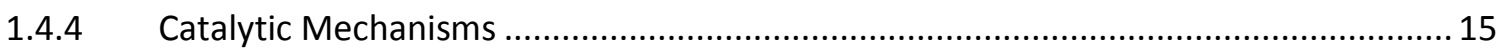

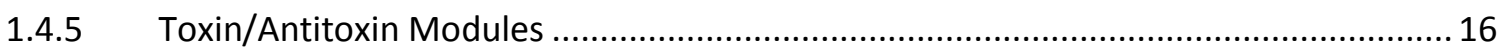

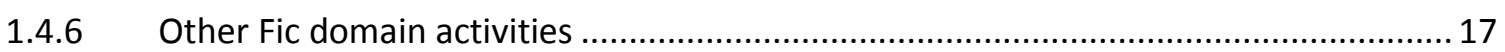

1.4.7 Role of Bartonella henselae BepA Fic Domain ......................................................... 17

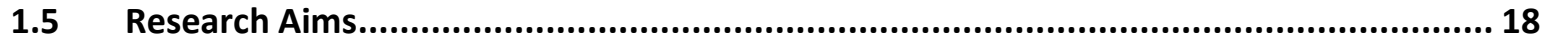

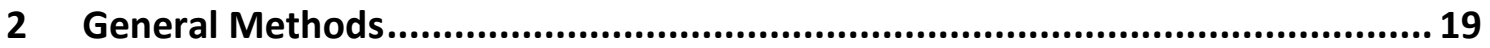

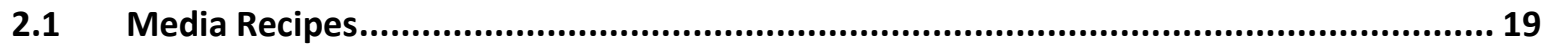

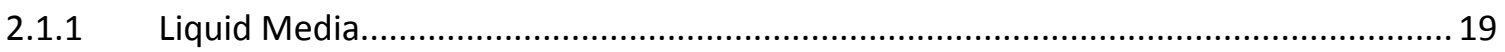

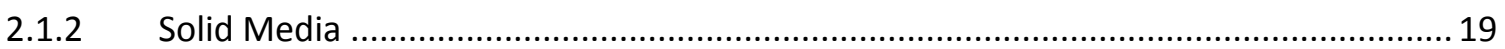




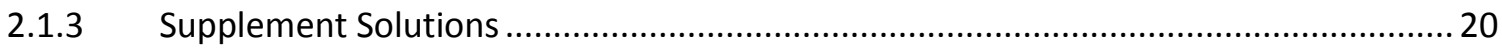

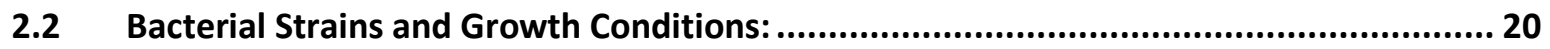

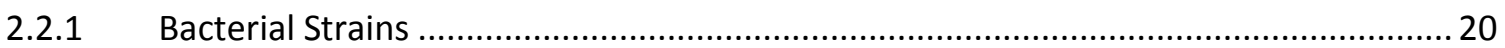

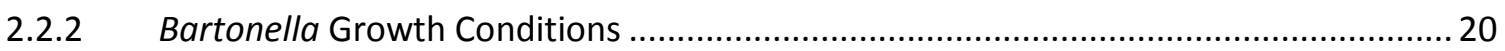

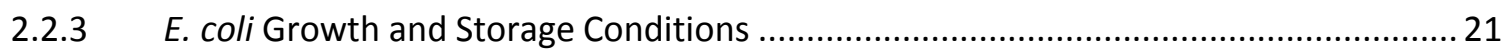

2.3 Plasmid Transformation of Competent Cells................................................................ 21

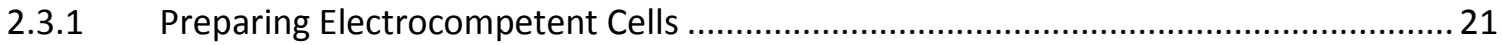

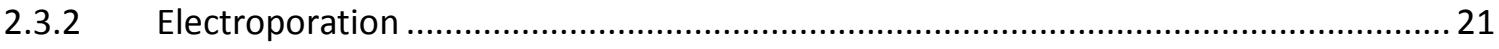

2.3.3 Transforming Chemically Competent Cells .................................................................... 22

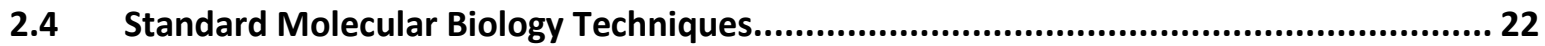

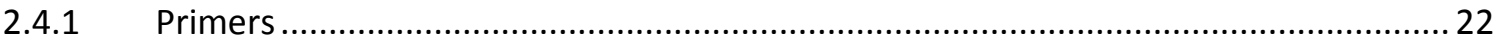

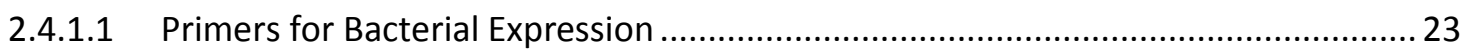

2.4.1.2 Primers for Expression in Mammalian Cells................................................................ 23

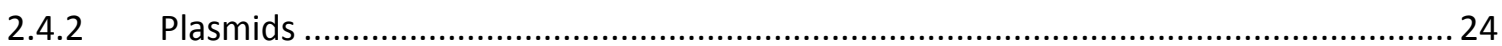

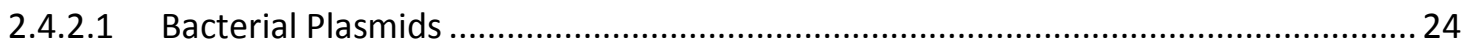

2.4.2.2 Plasmids for Mammalian Expression .................................................................... 24

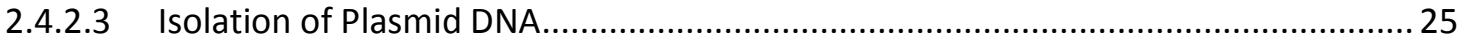

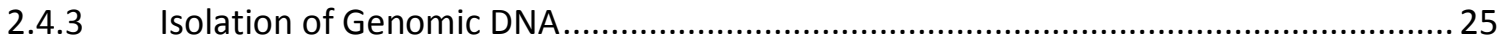

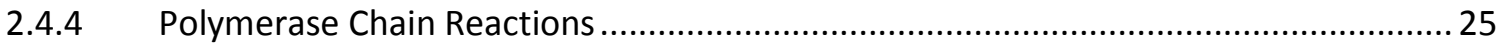

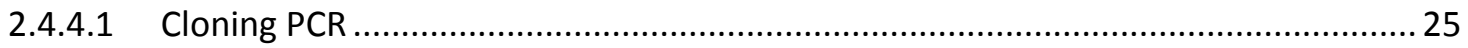

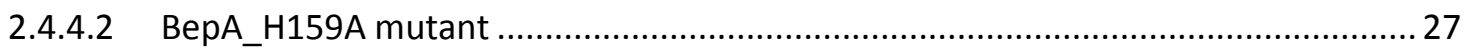

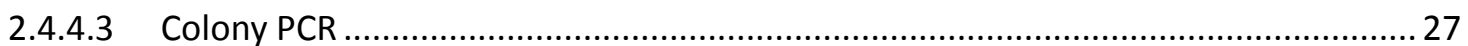

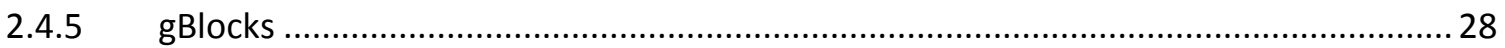

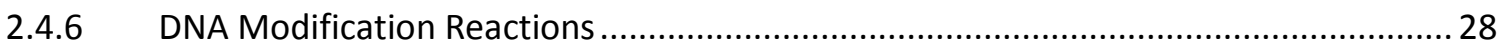

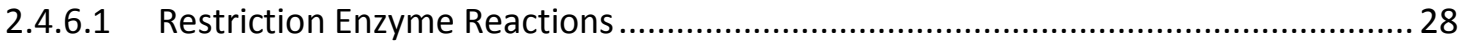

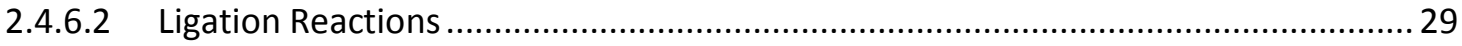

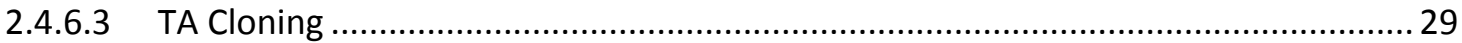

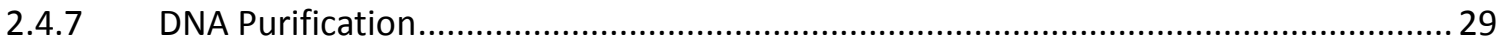

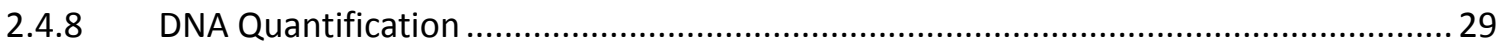

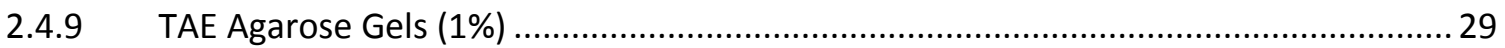

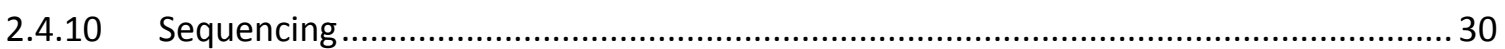

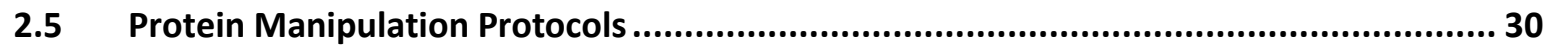

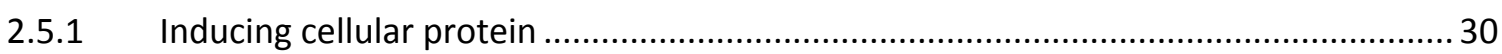

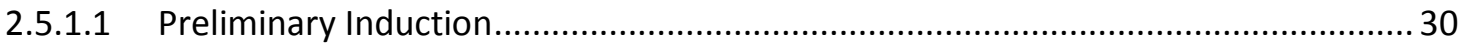




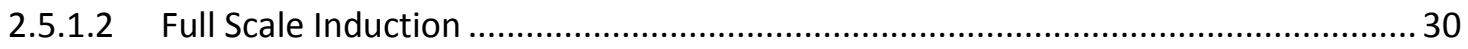

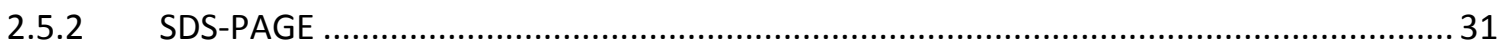

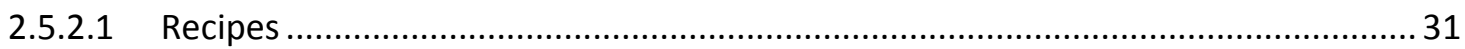

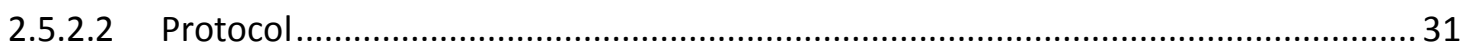



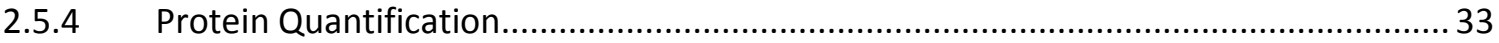

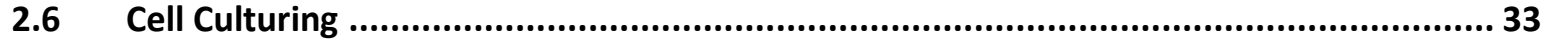

2.6.1 Initiation and Maintenance of HUVEC Cell Culture …................................................... 33

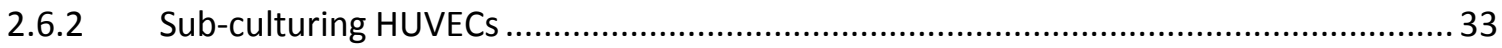

2.6.3 Initiation and Maintenance of HeLa Culture............................................................. 34

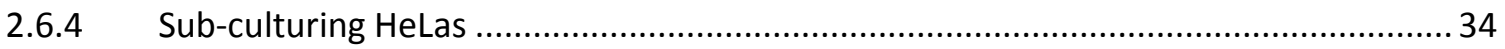

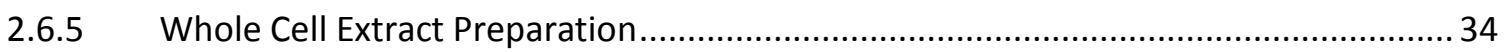

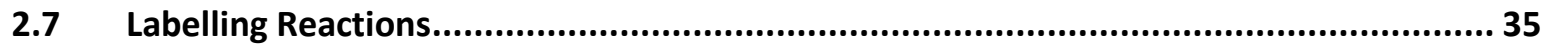

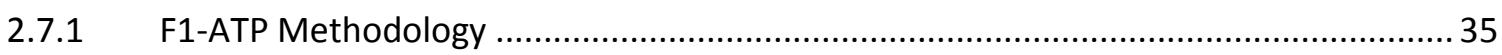

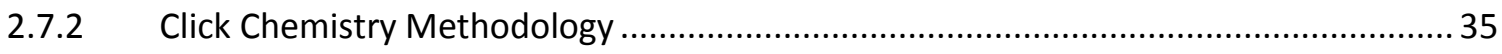

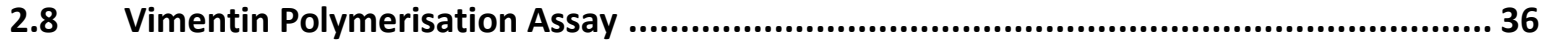

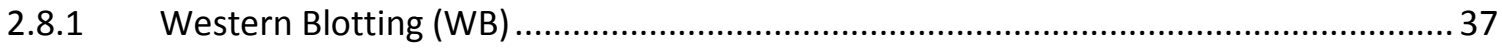

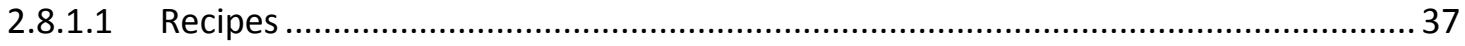

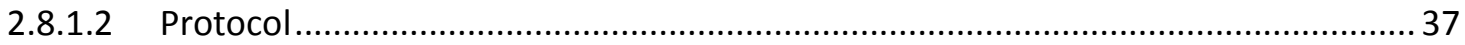

2.9 Pyrophosphate Detection/Bep activity assay ............................................................... 38

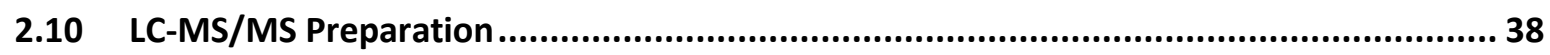

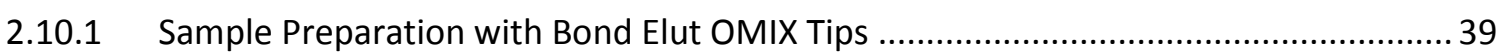

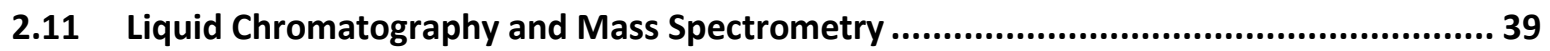

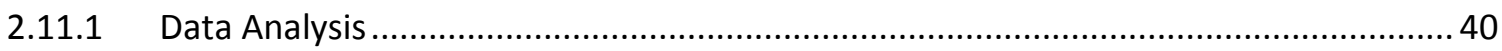

3 Labelling Whole Cell Extract with F1-ATP ........................................................ 41

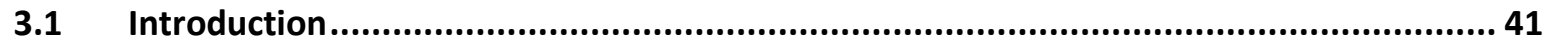

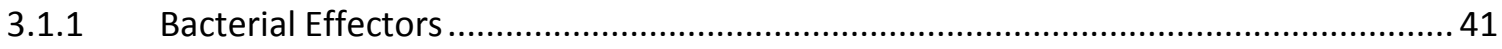

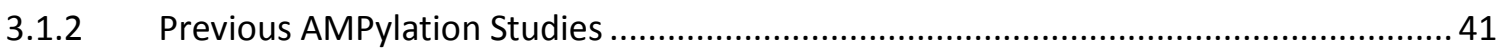

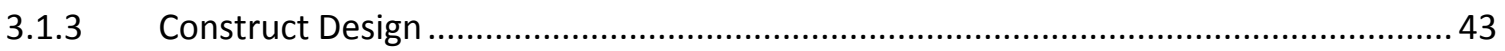

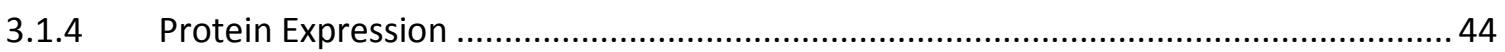

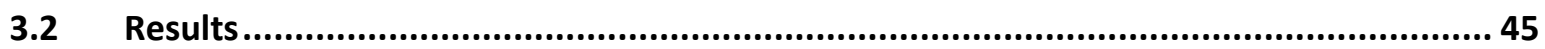

3.2.1 Optimisation of Protein Recovery from Whole Cell Extract .......................................... 45

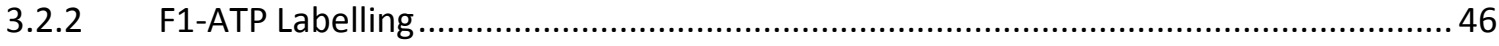

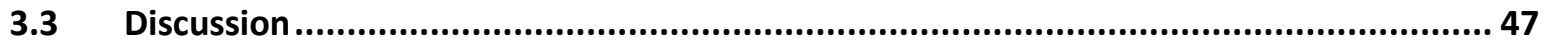


3.3.1 Protein Solubility

4 Click Chemistry 50

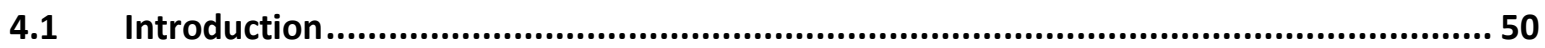

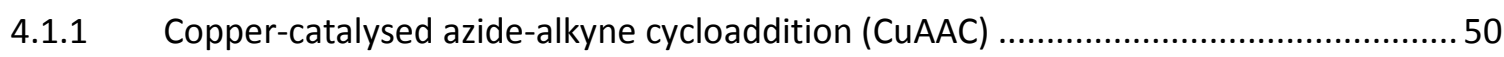

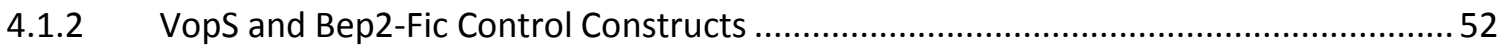

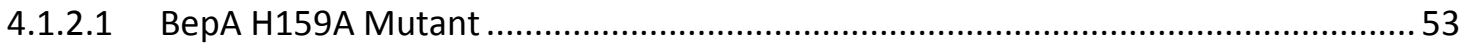

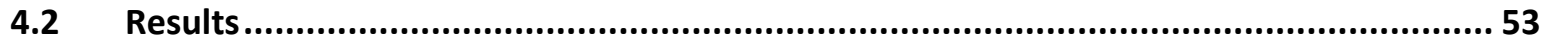

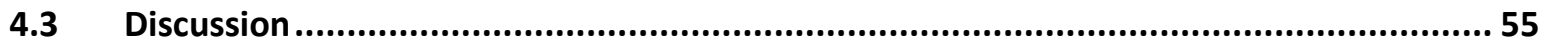

5 Vimentin, Pyrophosphate, and MS Assays...................................................56

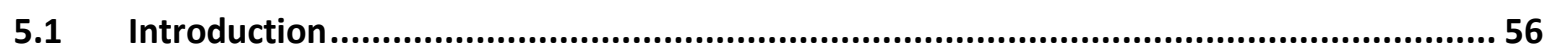

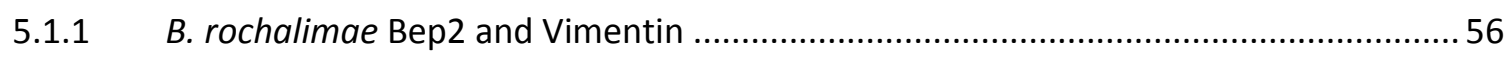

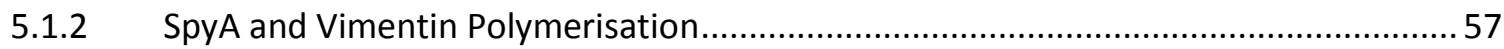

5.1.3 Pyrophosphate Analysis and Mass Spectrometry........................................................ 58

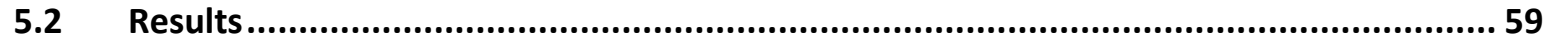

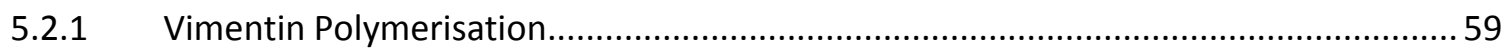

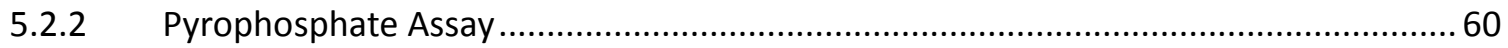

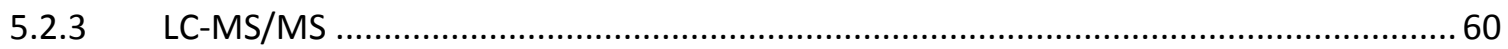

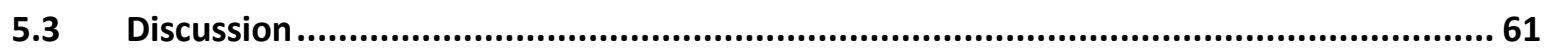

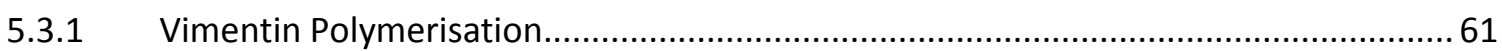

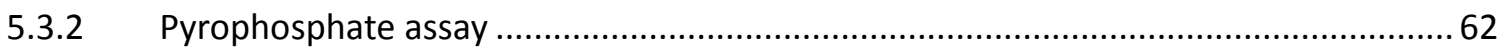

6 Research Motivation, Key Findings, and Future Directions............................63

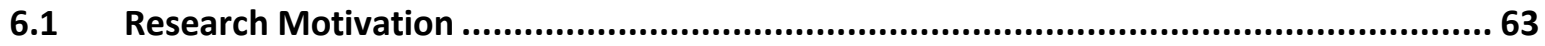

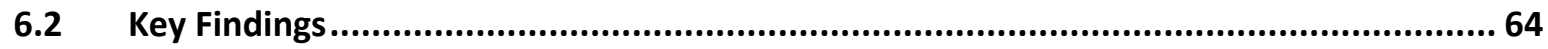

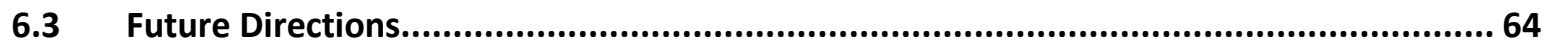

6.3.1 Improving Recombinant Protein Purification .................................................................... 64

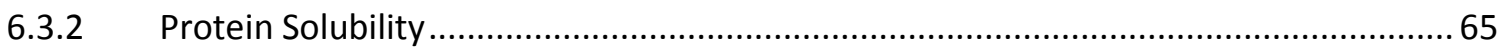

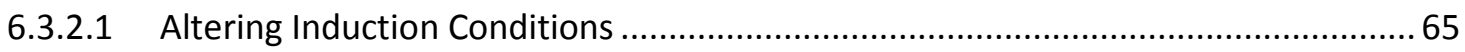

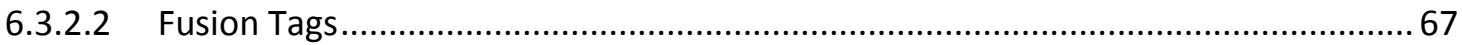

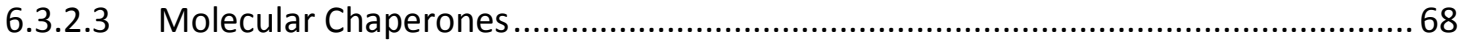

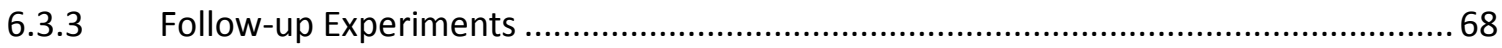

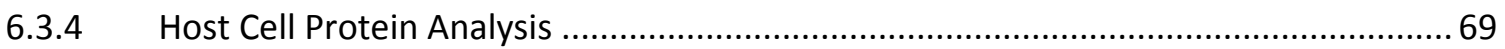

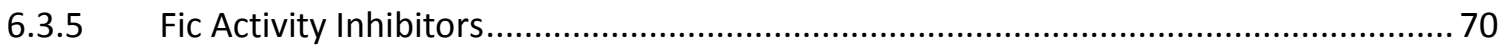

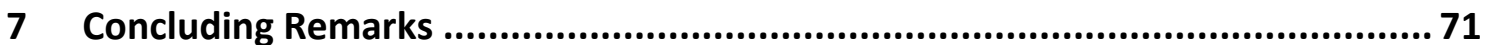

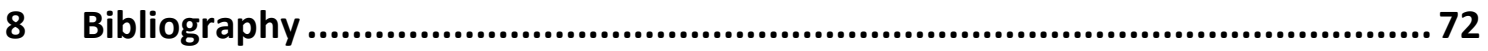


9 Appendix.

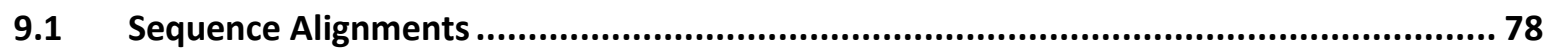

9.1.1 Bartonella henselae BepA-Fic H159A mutant design .................................................. 78

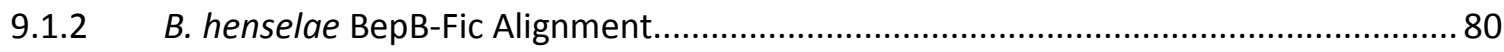

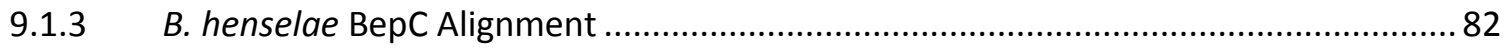

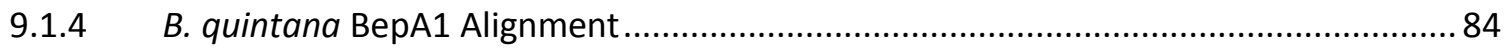

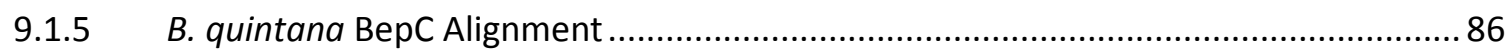

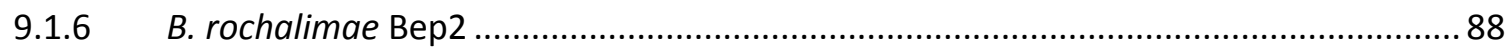

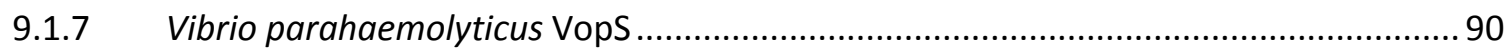

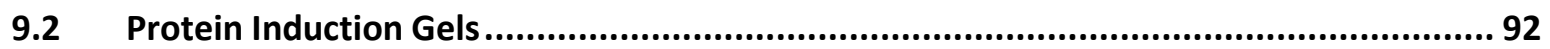

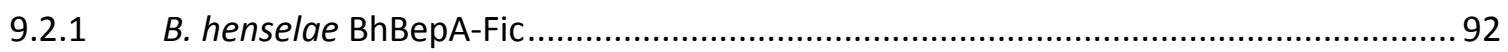

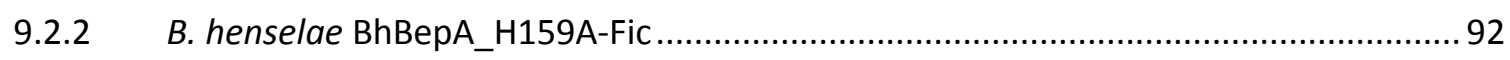

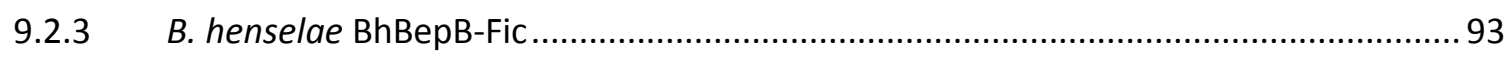

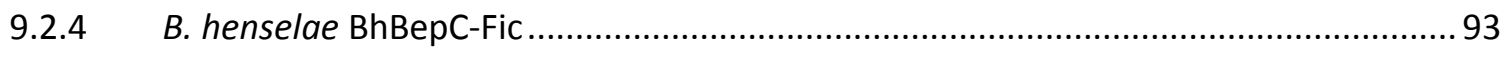

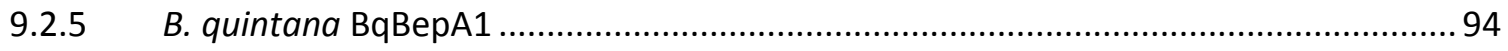

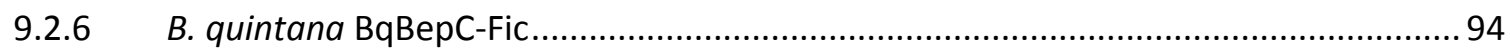

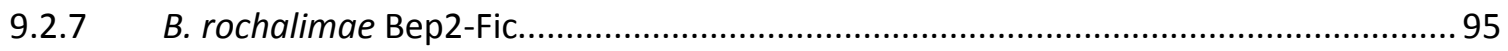

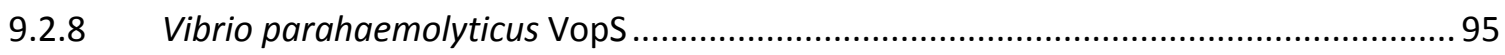

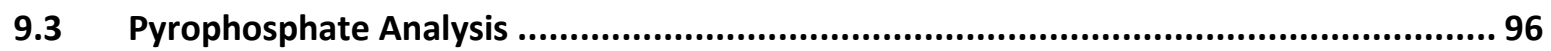




\section{List of Tables and Figures}

Figure 1.1: Infection strategy commonly employed by the bartonellae. ............................................ 7

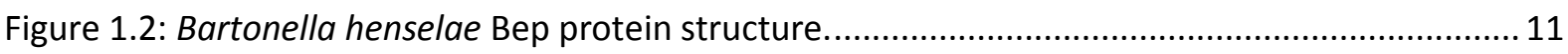

Figure 1.3: Proposed mechanism of VopS catalysed AMPylation of Cdc42 ....................................... 15

Figure 1.4: Proposed mechanism of IbpAFic2 catalysed AMPylation of Cdc42 .................................. 16

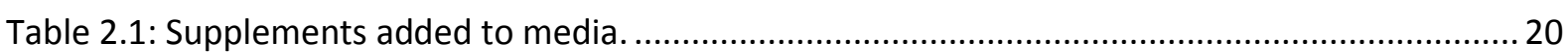

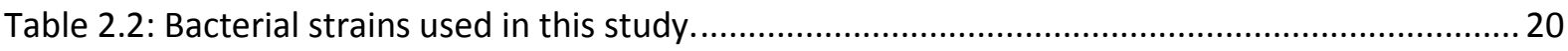

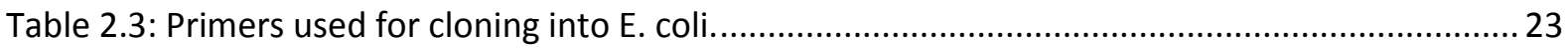

Table 2.4: Primers used for cloning mammalian cell expression plasmids. ........................................ 23

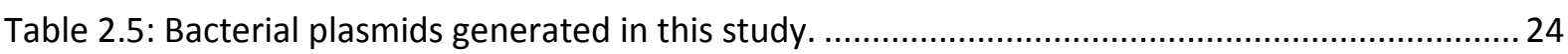

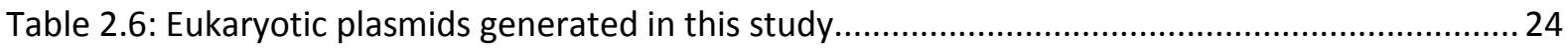

Table 2.7: Reaction components for PCR with Advantage Polymerase. ….......................................... 25

Table 2.8: Reaction conditions used for Advantage PCR with Bep-Fic primers.................................. 26

Table 2.9: Reaction components for PCR with Phusion Polymerase................................................. 26

Table 2.10: Reaction conditions used for Phusion PCR for BepC-Fic constructs................................26

Table 2.11 Site directed mutagenesis for BhBepA_H159A-Fic construction.......................................2 27

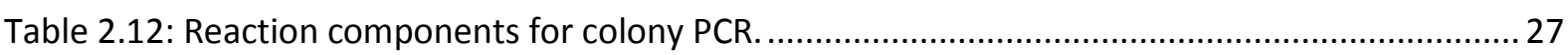

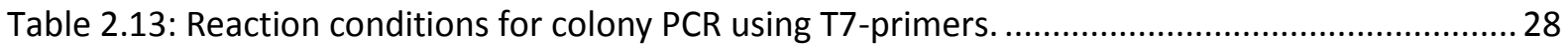

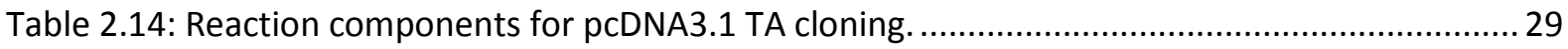

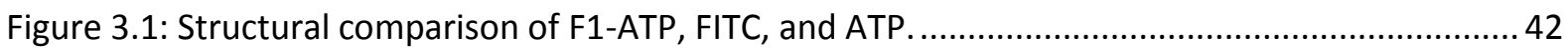

Figure 3.2: Bartonella effector proteins studied. ……..................................................................... 44

Figure 3.3: Optimisation of protein extraction from HUVEC cells. ....................................................... 45

Figure 3.4: Simplified methodology of the F1-ATP labelling experiments. ......................................... 46



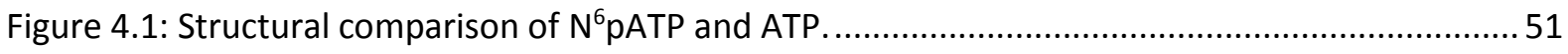

Figure 4.2: Basic reaction scheme for the CuAAC click chemistry reaction...................................... 52

Figure 4.3: Simplified methodology of the click chemistry labelling experiments...............................53

Figure 4.4: Results from the Click Chemistry Experiment.................................................................. 54

Figure 5.1: Cladogram of protein sequences for major Bartonella Bep proteins................................ 57

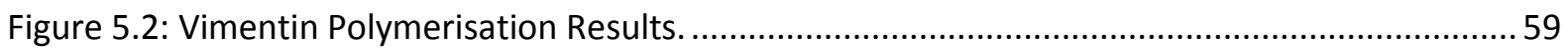

Appendix Figure 9.1: BhBepA-Fic H159A mutant sequence alignments.......................................... 79

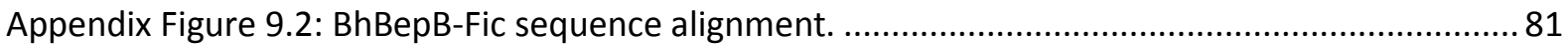




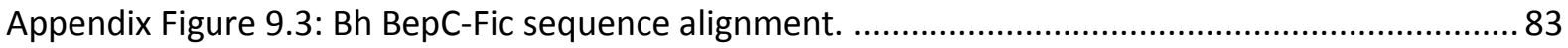

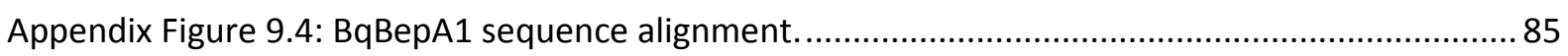

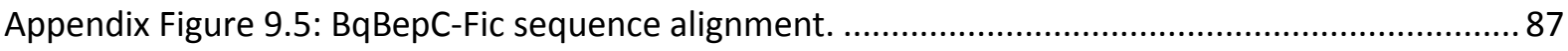

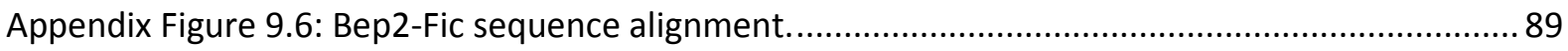

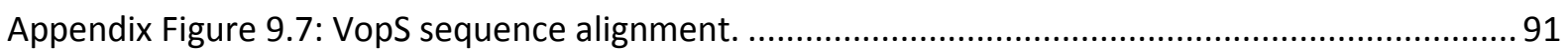

Appendix Figure 9.8: Nickel column affinity purification of BhBepA-Fic protein. ............................... 92

Appendix Figure 9.9: Nickel column affinity purification of BhBepA_H159A-Fic. ............................... 92

Appendix Figure 9.10: Nickel affinity purification of BhBepB-Fic protein. ......................................... 93

Appendix Figure 9.11: Nickel affinity purification of BhBepC-Fic protein. ........................................ 93

Appendix Figure 9.12: Nickel affinity purification of BqBepA1 protein............................................... 94

Appendix Figure 9.13: Nickel affinity purification of BqBepC-Fic protein. ......................................... 94

Appendix Figure 9.14: Nickel affinity purification of BrBep2-Fic protein...........................................95

Appendix Figure 9.15: Nickel affinity purification of VopS protein. .................................................. 95

Appendix Figure 9.16: Pyrophosphate reactions for $B$. henselae Fic proteins on vimentin samples...96

Appendix Figure 9.17: Pyrophosphate reactions for B. henselae Fic proteins on WCE samples. ........ 96 


\section{Abbreviations}

ACN - Acetonitrile

APS - Ammonium persulfate

ATCC - American Type Culture Collection

Bep - Bartonella Effector Protein

BID - Bartonella Intracellular Delivery

CTAB - cetyltrimethylammonium bromide

CSD - Cat-Scratch Disease

$\mathrm{dd}_{2} \mathrm{O}$ - Double distilled water

DMSO - Dimethyl sulfoxide

dNTP - Deoxynucleoside triphosphate

DTT - Dithioreitol

EDTA - Ethylenediaminetetraacetic acid

F1-ATP - N6-(6-amino)-hexyl-ATP-5-FAM

FAM - Fluorescein amidite

FCS - Fetal Calf Serum

Fic - Filamentation induced by cyclic AMP

FITC - Fluorescein isothiocyanate

GFP - Green fluorescent protein

HEPES - N-2-hydroxyethylpiperazine-N'-2-ethanesulfonic acid

HUVEC - Human Umbilical Vein Endothelial Cell

IPTG - Isopropyl-1-thio- $\beta$-D-galactoside

LC-MS/MS - Liquid Chromatography-Mass Spectrometry/Mass Spectrometry

MES - 2-(N-morpholino)ethanesulfonic acid

$N^{6}$ pATP - N6-Propargyl-Adeonsine-5'-triphosphate

$\mathrm{NCBI}$ - National Centre for Biotechnology Information

$\mathrm{OD}_{600}$ - Optical Density at $600 \mathrm{~nm}$

PAGE - PolyAcrylamide Gel Electrophoresis

Penstrep - Penicillin Streptomycin

PCR - Polymerase Chain Reaction

RIPA - RadiolmmunoPrecipitation Assay

SDS - Sodium dodecyl sulfate

T4SS - Type IV Secretion System

TCA - Trichloroacetic acid

TBS - Tris-buffered saline

TBS-T - TBS-Tween

TE - Tris/EDTA

TEMED - N,N,N', $\mathrm{N}^{\prime}$-tetramethylethylenediamine

THPTA - Tris(3-hydroxypropyltriazolylmethyl)amine

Tris - Tris(hydroxymethyl)aminomethane 


\section{Introduction}

\subsection{Bartonella Species and Associated Disease}

\subsubsection{Bartonella}

Newly emerging or re-emerging infectious diseases are a significant global health concern. A recent study found that, of pathogens that have been newly recognised since $1940,60 \%$ are associated with zoonotic diseases, and $54 \%$ are of a bacterial or rickettsial origin ${ }^{1}$. The Bartonellae represent an increasing number of emerging zoonotic bacterial pathogens. Prior to 1990, only two Bartonella species and their associated disease were known: Rochalimae quintana, the causative agent of Trench Fever during World War I and $\mathrm{II}^{2-4}$, and B. bacilliformis, the causative agent of Carrión's disease in Peru'2,3,5,6. Bartonella species have since reappeared as significant opportunistic infections during the AIDS epidemic, and have been subsequently identified and characterised. Through analysis of $16 \mathrm{~S}$ ribosomal DNA sequences, the causative agent of cat-scratch disease (CSD) was found to be highly related to R. quintana ${ }^{7}$. This unknown organism was later identified as $R$. henselae ${ }^{8}$. Following this discovery, the genus Rochalimaea was unified with Bartonellae $e^{9}$ and has subsequently grown rapidly to $30-40$ currently recognised species of Bartonella ${ }^{3,10}$, at least seventeen of which have been reported as human pathogens ${ }^{11}$.

The genus Bartonellae consists of fastidious, Gram-negative, rod-shaped bacteria ${ }^{12-14}$, that behave as facultative intracellular pathogens ${ }^{15}$. With the exception of $B$. bacilliformis, most Bartonellae are both microaerobic and capnophilic ${ }^{3,16}$, requiring high $\mathrm{CO}_{2}$ conditions for growth. Bartonella species utilise a relatively unique infection strategy whereby they alternate between mammalian and arthropod hosts, invading their mammalian host's erythrocytes and establishing a persistent infection ${ }^{10,12,15,17}$. This ability to establish an intraerythrocytic infection defines the mammalian host as a reservoir host, and infection typically results in asymptomatic bacteraemia ${ }^{3,15}$. Incidental hosts are those that are infected by a Bartonellae species from a different reservoir (e.g. CSD in humans). Intraerythrocytic infection does not occur in incidental hosts, and the result may be either a self-limiting or a chronic infection ${ }^{11,15,17,18}$. 
B. bacilliformis was the first described member of the genus and is spread through the bite of the sand fly, Lutzomyia verrucarum, found in Peru, Columbia, and Ecuador $2,3,5,6,13,17$. The geographical restriction of the sand fly is believed to be the reason for the localised, endemic nature of $B$. bacilliformis infections ${ }^{2}$. Infection can result in a life threatening haemolytic anaemia known as Oroya fever, which has a $40-90 \%$ mortality rate if left untreated, and occurs during the acute phase of the infection ${ }^{3,15}$. In the chronic stages of the infection multiple vasoproliferative skin lesions, the verruga peruana, are formed ${ }^{2,6,15}$. These lesions are characterised by lobular proliferations of atypical endothelial cells ${ }^{2}$. This chronic phase of infection is associated with a much lower mortality than the acute phase $\mathrm{e}^{15}$. Humans are the only known reservoir host ${ }^{2,15}$.

Bartonellosis, a term originally used to describe this disease, now includes a range of human diseases associated with Bartonella infection; most of which have a much lower severity than Oroya fever ${ }^{2,3}$. Bartonellosis conditions can involve multiple combinations of bacteraemia, low-grade fever, malaise, culture-negative endocarditis, vascular lesions of the skin, and or liver, as well as lymphadenopathy, although these conditions usually only occur in immunocompromised individuals ${ }^{3}$.

Similarly, Bartonella infections have been linked to endocarditis in both cats and dogs including cases reported from both New Zealand and Australia ${ }^{14}$. While infections are usually self-limiting, there have been more severe clinical manifestations associated with Bartonella infection including; encephalitis, neuroretinitis, anterior uveitis, haemolytic anaemia thrombocytopenia, glomerulonephritis, pneumonia, and osteomyelitis ${ }^{2,19}$. Bacteraemia is also observed in many mammalian species infected with Bartonella, and as sensitivity of diagnostic methods improves, there is increasing evidence that many immunocompetent individuals are chronically infected with Bartonella as incidental hosts $5,11,15,19-22$. People with occupational exposure to animals or biting arthropods, including veterinarians, avid hunters, or wildlife biologists, appear to be most at risk of chronic cryptic Bartonella infections. Currently, blood donors are not routinely screened for Bartonella, and studies have shown that the bacteria can survive the storage conditions for erythrocytes ${ }^{23,24}$. The incidence of Bartonella infection acquired through blood transfusion is not known. The three most common pathogenic strains that infect humans are B. bacilliformis, $B$. quintana, and $B$. henselae ${ }^{2,15}$ with $B$. henselae being the most prevalent ${ }^{15}$. 


\subsubsection{Bartonella quintana}

B. quintana was first identified as a widespread pathogen among soldiers during World War I, causing a disease that became known as trench fever $2,3,6,13,15$. Trench fever is estimated to have affected over one million Allied soldiers on the Western Front with an average weekly loss of $0.6 \%$ army strength in France, from evacuation due to sickness ${ }^{25}$. However, B. quintana infections from long before this have been documented, as Drancourt et al. (2005) identified B. quintana DNA from a human tooth dating back at least 4000 years. Trench fever, also known as five-day fever ${ }^{4}$, is characterised by relapsing peaks of fever, headache, and bone pain $^{15}$, particularly in the long bones of the legs ${ }^{2,3,13}$. The incubation period for the disease is between 15 - 25 days and symptoms typically range from mild to severe, although some individuals may be asymptomatic ${ }^{3,4,13}$. Infections are accompanied by consistent bacteraemia and, though most infections are usually self-limiting, 5-10\% of sufferers may remain infectious for months to years after the initial infection ${ }^{2,13,15}$. In immunocompromised individuals, $B$. quintana can also cause bacillary angiomatosis as well as endocarditis, an infection of the heart valves $2,4,13,15$. Bacillary angiomatosis describes the outgrowth of multiple vasoproliferative tumours that can be found on the skin and on other organs of the body, and are similar to the verruga peruana lesions associated with $B$. bacilliformis ${ }^{13,15}$. These skin lesions usually present as pink to deep red or purple papules or nodules ${ }^{2,13}$. After reappearing during World War II, the incidence of trench fever decreased below detectable levels, before re-emerging with the AIDS epidemic as urban trench fever ${ }^{2,15}$. This condition is associated with homelessness, alcoholism, and other conditions of poor hygiene ${ }^{2,15}$. Bartonella quintana is mainly spread by the body louse, Pediculus humanus corporis, although other vectors have been reported ${ }^{2,15}$. Individuals who wear unwashed clothing for long periods are susceptible to louse infestation and therefore infection by Bartonella quintana. Like B. bacilliformis, humans are the main reservoir ${ }^{4,6}$. 


\subsubsection{Bartonella henselae}

Cat scratch fever, later renamed cat scratch disease (CSD), was first described in 1950, but was thought to be caused by a viral agent, as no bacteria could be observed or cultured ${ }^{27}$. Bartonella henselae was later discovered and isolated from immunocompromised patients in 1990 , through the use of $\mathrm{PCR}^{7}$ and blood cultures ${ }^{28}$. It wasn't identified however, until it was re-isolated from the blood of an HIV positive patient in $1992^{8}$. The reservoir host for $B$. henselae is the cat, either domestic or feral, and humans are an incidental host ${ }^{2,13,15}$. Humans are typically infected by cat scratches or bites, resulting in a mild, self-limiting illness known as CSD. The infection is transmitted between cats via an arthropod vector, the cat flea (Ctenocephalides felis) $^{2,13}$. There is however, no conclusive evidence to suggest flea bites can transmit the bacteria to humans ${ }^{2}$. Studies in New Zealand have shown that $11-17 \%$ of cats in a given region may be infected ${ }^{29,30}$. Evidence has also shown that $B$. henselae infects feline erythrocytes, however there is controversy as to whether it naturally infects human erythrocytes like B. bacilliformis and B. quintana ${ }^{31,32}$.

CSD is characterised mainly by lymphadenopathy, enlarged tender lymph nodes, and is usually accompanied by fever $2,3,5,15$. In immunocompromised people, complications from $B$. henselae can include bacillary angiomatosis, endocarditis, and bacillary peliosis. Bacillary peliosis describes the formation of lesions on the liver or spleen and is very similar to bacillary angiomatosis. One study in New Zealand found $5 \%$ of blood donors tested were seropositive for $B$. hensela $e^{33}$. There is however, increasing evidence that many bacteraemic individuals have negative serology results for Bartonella species $5,11,21,34,35$. This has been discovered following the development of improved tests for Bartonella, such as PCR analysis, and BAPGM (Bartonella alpha Proteobacteria growth medium) to allow for enrichment culture of Bartonella from bacteraemic individuals $11,21,34,36$. This challenges the notion that bacteraemia is rare in immunocompetent individuals, and suggests that the prevalence of $B$. henselae bacteraemia is much higher than expected, particularly in those working closely with animals $s^{5,11,19-22}$. There is also increasing evidence that many of these individuals are coinfected chronically with multiple species of Bartonella $a^{5,11,21,34,36}$. This has the potential to allow horizontal gene transfer between different Bartonella species to occur ${ }^{37}$. 


\subsubsection{The Bartonella Genome}

Bartonellae species share similarities between their genomes, such as low GC count (38\%), and similar numbers of tRNA and rRNA molecules ${ }^{38}$. There are also however, some major differences even between closely related species. Historically, the genus Bartonellae has been divided into 4 major lineages ${ }^{15,37,38}$. Lineage 1 describes an ancestral lineage and is the most diverged from the remaining lineages. This lineage contains only 1 species, B. bacilliformis, which contains the smallest genome of all the currently sequenced Bartonella species (1.4 $\mathrm{Mbp}^{38}$. Conversely, Lineage 4 is very species rich and contains the most diverse group of species of any of the lineages ${ }^{38}$. Recently, this lineage model has been challenged, as new sequence data and phylogeny analysis have found that $B$. australis, a species of Bartonellae that infects kangaroos, appears to have diverged earlier than B. bacilliformis, which clustered with the ruminant-infecting Bartonella that make up Lineage $2^{37}$. It is proposed that, rather than being an ancestral strain, B. bacilliformis arose from reductive genomic evolution following a host switch. Based on these phylogenetic analyses, three Bartonellae groups were proposed: Group A, consisting of the Lineage 2 Bartonellae, Group B consisting of the Lineage 3 species, and Group C, consisting of Lineage $4 \operatorname{species}^{37}$. B. australis has not currently been assigned a lineage or group.

Both $B$. henselae and $B$. quintana are members of Group $C$ and genomic studies have shown they are very closely related ${ }^{38,39}$. In spite of this, the genomes of these two species differ in size. B. henselae has one of the largest Bartonella genomes currently sequenced (1.9 Mbp), while $B$. quintana has a much smaller genome $(1.5 \mathrm{Mbp})^{38,39}$. Interestingly, B. quintana has 67 pseudogenes as well as many gene remnants that are still expressed in $B$. henselae, whereas B. quintana has 6 functional homologues of $B$. henselae pseudogenes. B. henselae also contains a prophage region that encodes $62 \%$ of the genes not expressed in B. quintana, which only has gene remnants of this prophage, suggesting it was lost during B. quintana evolution. The differences in the genomes of the two species have led researchers to believe that the $B$. quintana genome is derived from a larger ancestral $B$. henselae-like genome by reductive genomic evolution ${ }^{39}$. 


\subsubsection{Bartonella Lifecycle}

Bartonella species typically alternate between a warm-blooded host and an arthropod vector. When an infected haematophagous arthropod takes a blood meal, Bartonellae are shed in the faeces and are inoculated when the host scratches the bacteria into the $\operatorname{skin}^{17}$. This results in bacterial infection of the dermal niche, with evidence suggesting dermal dendritic cells (DCs) are infected ${ }^{40}$. Following dermal niche colonisation, it is thought that the bacteria induce infected DCs to migrate to the bloodstream, where Bartonella subsequently colonise the blood seeding, or primary, niche. This blood seeding niche is hypothesised to be endothelial cells or mononucleated cells of the reticulo-endothelial system, although this is still heavily debated $15,17,40,41$. Five days after infection, the bacteria escape from the blood seeding niche and enter the bloodstream, where they invade the reservoir host's erythrocytes ${ }^{15,17}$. The bacteria are repeatedly, synchronously, released from the blood seeding niche every few days giving rise to a recurring fever ${ }^{17}$. The infection of erythrocytes involves three main stages: adhesion, invasion, and intracellular persistence ${ }^{15}$. Bartonellae replicate inside erythrocytes until a critical population density is reached ${ }^{17}$. At this point, the number of bacteria remains static for the remainder of the erythrocyte's lifespan and these infected erythrocytes function essentially normally ${ }^{17}$. This infection of erythrocytes enables the bacteria to persist in an immunologically privileged site ${ }^{12}$, and promotes transmission by the haematophagous arthropod vector ${ }^{17}$ (Figure 1.1). B. bacilliformis is an exception to this strategy, as it lyses erythrocytes after invasion, causing the potentially lethal haemolytic anaemia associated with Oroya fever ${ }^{2,15,17}$. 


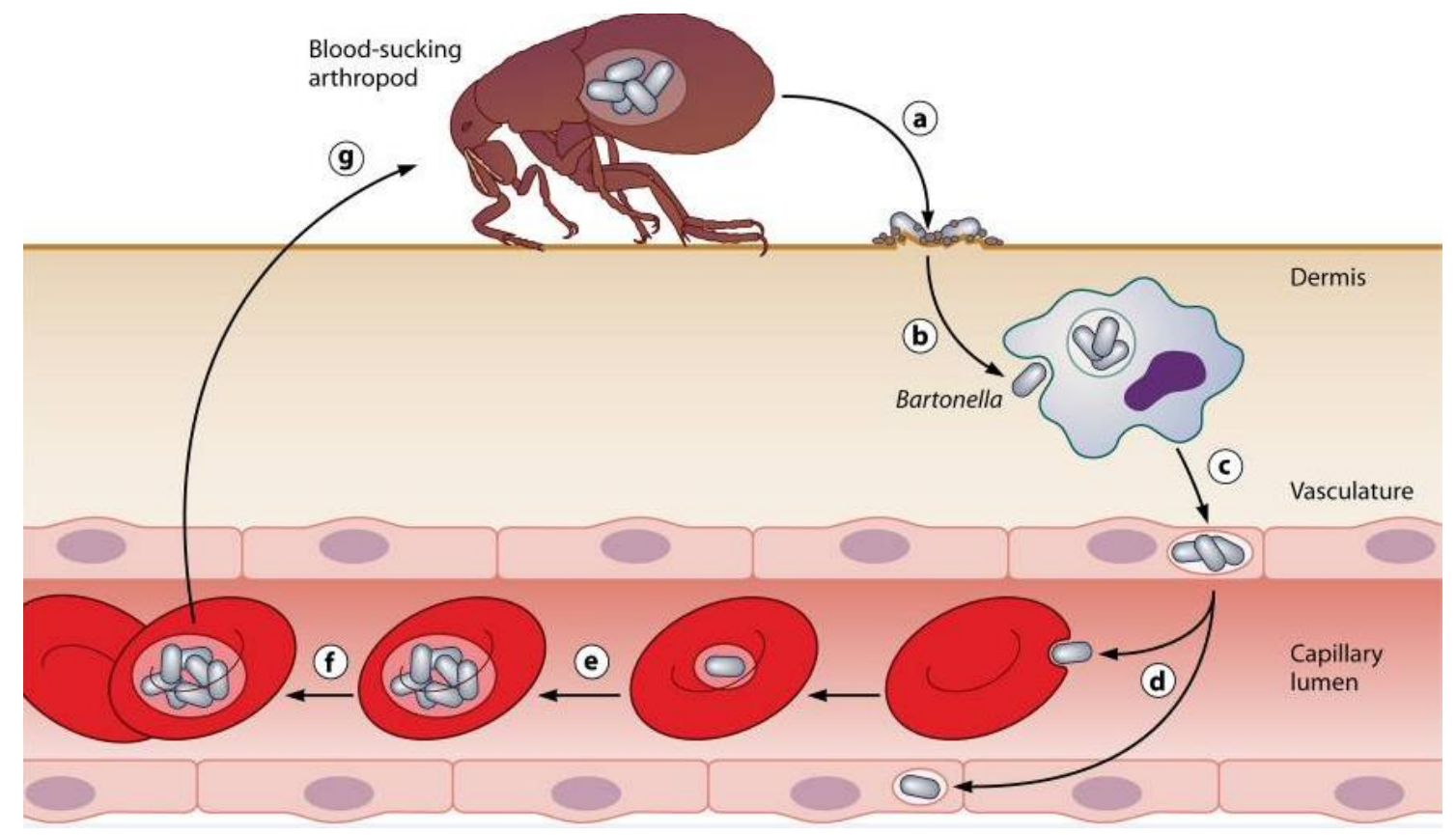

Figure 1.1: Infection strategy commonly employed by the bartonellae. (A) When an infected haematophagous arthropod takes a blood meal it inoculates the host, resulting in colonisation of the dermal niche. (B) In the dermal niche, bacteria are taken up by dendritic cells. (C) Bartonella are transported by dendritic cells to the blood-seeding niche. (D) Bacteria escape from the blood-seeding niche and enter the bloodstream where they colonise the host's erythrocytes and re-infect the bloodseeding niche. (E) Bartonella persist and replicate inside the host erythrocytes until a critical population is reached (F). (G) Infected erythrocytes are taken up by a haematophagous arthropod for further transmission. Reproduced with permission from The American Society for Microbiology. Harms \& Dehio $(2012)^{15}$.

\subsubsection{Bartonella and Endothelial Cells}

Bartonella species are able to colonise endothelial cells resulting in vasoproliferative tumour growth, particularly in immunocompromised patients ${ }^{41}$. This is believed to be a crucial step in the formation of verruga peruana, bacillary angiomatosis, and bacillary peliosis syndromes. Experiments using both live bacteria and cell free extracts from the three Bartonella species detailed above stimulated the proliferation of human umbilical vein endothelial cells (HUVECS) in vitro ${ }^{41-43}$. In addition, B. quintana and B. henselae stimulated migration of HUVECs through a porous membrane, while B. bacilliformis was angiogenic in vivo in a rat model $^{41,42}$.

Experiments with $B$. bacilliformis have shown that activation of the host Rho GTPase is involved in internalisation of the bacteria ${ }^{44}$, and it is believed that both $B$. quintana and $B$. henselae utilise a similar mechanism ${ }^{41}$. This activation results in the formation of actin stress fibres, which are associated with altered cell morphology, impaired migration, and defects in cell morphogenesis in HUVEC primary cells ${ }^{41,45}$. The invasion process is actin-dependent, occurs in a range of hours, and results in membrane bound clusters of bacteria ${ }^{41}$ reminiscent of bacterium-directed phagocytosis described in other bacterial species ${ }^{46-50}$. 
In addition to this mechanism, $B$. henselae uses a second method for internalisation. The bacteria form aggregates on the endothelial cell surface that contain hundreds of bacteria. These aggregates are engulfed by plasma membrane-derived protrusions, eventually resulting in their internalisation in a host structure: the invasome ${ }^{41,51}$. In Bartonella henselae, invasome formation is dependent on host cell proteins including integrin $\beta 1$ receptor on the cell surface and talin-1 activation inside the cell ${ }^{52}$. Talin- 1 is required to bind integrin $\beta-1$ and to stabilise it in an open conformation allowing $B$. henselae to translocate effector proteins (discussed below) into the endothelial cells.

\subsection{Bartonella Virulence Factors}

Bartonella species possess a wide range of virulence factors. These include trimeric autotransporter adhesins, such as Bartonella adhesion A (BadA) in B. henselae, or the four variable outer membrane proteins (Vomps) in B. quintana, which mediate adhesion to the host cell extracellular matrix ${ }^{53,54}$. BadA has been reported to be responsible for activation of hypoxia inducible factor 1 (HIF-1), and vascular endothelial growth factor (VEGF) secretion in macrophages and epithelial cells ${ }^{55}$. B. henselae also expresses filamentous hemagglutinins which mediate adhesion in other gram-negative bacteria, but whose function is not known in B. henselae. Many Bartonella species also possess flagella which appears to have been lost in Group $C^{15,37,56}$.

B. quintana and B. henselae also encode two different type IV secretion systems (T4SSs). The first is the Trw T4SS, which forms surface pilus structures and has been shown to play a role in the colonisation of host erythrocytes ${ }^{15,56}$. The Trw T4SS, as the bacterial factor that enables erythrocyte infection, also determines host specificity, as Bartonella are only able to invade erythrocytes from their reservoir host. Identification of Trw as a key component in hostspecific adhesion for erythrocytes has enabled the identification of its target, band3, a major outer membrane glycoprotein of erythrocytes ${ }^{57}$. Only Bartonella from Group C possess the Trw T4SS, which appears to be correlated with a loss of flagella in these Bartonella species $^{15,37,53,56}$. The other critical T4SS of Bartonella is the VirB/D4 system, which secretes a suite of $\underline{B} a r t o n e l l a$ effector proteins (Beps) directly into the host cell ${ }^{15}$. This system is possessed by Bartonella species from both Groups B and C, with Group B containing multiple copies $^{15,37}$. 
Experiments using the rodent-adapted $B$. tribocorum have demonstrated the importance of these T4SSs, with mutations in either the VirB4, VirD4, or Trw proteins resulting in complete loss of erythrocyte infection in a rat model ${ }^{58,59}$.

\subsubsection{VirB/D4 T4SS and Secreted Effector Proteins}

T4SSs are macromolecular machineries that inject bacterial DNA or proteins directly into a host cell and are widely expressed in bacterial pathogens, having evolved from conjugation machinery ${ }^{15}$. They form a translocation channel that spans the inner and outer membranes of Bartonella and the host cell cytoplasmic membrane ${ }^{15}$. T4SSs typically exert their pathogenesis through the secretion of effector proteins directly into the host cell by a poorly understood mechanism.

The VirB/D4 T4SS is found in both Group B and Group C species of Bartonella, including B. henselae and B. quintana ${ }^{15,17,37}$. It has been proposed that the VirB/D4 genes were originally acquired by two independent horizontal gene transfers, giving rise to the observed differences between Group B and Group C Bartonella species ${ }^{37}$. However, gene transfers still frequently occur in this region between different Bartonella species, particularly during coinfection. Evidence for this is found in the $B$. vinsonii strain Winnie, isolated from a dog that had been co-infected with $B$. henselae. Phylogenetic analysis found that the VirB genes (virB2 - virB8) in this strain of B. vinsonii, a dog-associated Bartonella species, were more closely related to $B$. henselae than $B$. vinsonii. Conversely, the VirB genes (virB9-virD4) were more closely related to those of $B$. vinsonii VirB/D4 genes ${ }^{37}$. This provides strong evidence that during co-infection a gene transfer event occurred. In contrast, the Trw secretion system is believed to have been acquired through a single horizontal gene transfer ${ }^{37}$.

The VirB/D4 T4SS contributes to virulence through its ability to translocate Beps directly into the host cell ${ }^{15,60}$. The Houston-1 strain of $B$. henselae ${ }^{8}$ elicits many VirB/D4-dependent phenotypes in endothelial cells, suggesting that the VirB/D4 T4SS is an important factor in the manipulation of endothelial cells during infection ${ }^{15}$. These phenotypes include activation of NF-KB and the pro-inflammatory response, inhibition of apoptosis, and capillary-like sprout formation of endothelial cell aggregates $3,51,53,61-63$. This system is also important in mediating rearrangements in the host cell actin cytoskeleton, which results in internalisation of bacterial aggregates, formed on the cell surface, in the invasome ${ }^{15,63}$. 
Evidence has emerged that suggests this system is also able to translocate a Bartonella plasmid into a human cell, however the significance of this during infection is not known ${ }^{64}$.

\subsection{Bartonella VirB/D4 Secreted Effector Proteins}

\subsubsection{Bep Protein Structure}

Both $B$. henselae and $B$. quintana contain a pathogenicity island that encodes the highly conserved VirB/D4 T4SS, as well as the secreted Bep proteins ${ }^{65}$. These effector proteins display a modular structure with an $\mathrm{N}$-terminal host interacting domain, and a C-terminal bipartite secretion signal. This bipartite signal consists of a roughly 142 amino acid Bep intracellular delivery (BID) domain followed by a non-conserved tail sequence with a net positive charge ${ }^{3,15,65}$. Three of the Bartonella henselae Bep proteins contain more than one BID domain (BepE $-G$ ), although it is the most C-terminal BID domain that is involved in translocation ${ }^{51,62}$. This suggests that the remaining BID domains may have functions other than translocation. The Bep proteins have a high degree of plasticity and are not completely conserved between the Bartonella species ${ }^{65}$.

Several of the Bep proteins found in both B. henselae and B. quintana contain an N-terminal filamentation induced by $\underline{\text { AMMP }}$ (Fic) domain ${ }^{15}$. This domain is usually followed by a small oligonucleotide/oligosaccharide binding fold and finally the secretion signal. B. henselae's pathogenicity island encodes 7 Bep proteins (BepA - G), three of which (BepA $-C$ ) are homologues and contain a Fic domain ${ }^{15,66}$. In contrast, BepD - F contain multiple N-terminal motifs for tyrosine phosphorylation. These motifs are characterised by the amino acid sequence Glu-Pro-Ile-Tyr-Ala (EPIYA) and have been shown to undergo tyrosine phosphorylation by endogenous host cell kinases. EPIYA motifs are believed to interact with $\mathrm{SH} 2$ domain-containing host cell proteins which are specific for phosphorylated tyrosine ${ }^{67}$. An example of this is BepF, which has been demonstrated to interact with the $\mathrm{SH} 2$ domaincontaining signalling and adaptor proteins Grb2, RasGAP, and $\mathrm{Crk}^{51,65}$. BepG differs from other Bep proteins, as it does not contain either of these $\mathrm{N}$-terminal motifs, but instead consists of four BID domains with short repeat sequences interspersed ${ }^{62}$ (Figure 1.2). 


\section{Bartonella henselae}

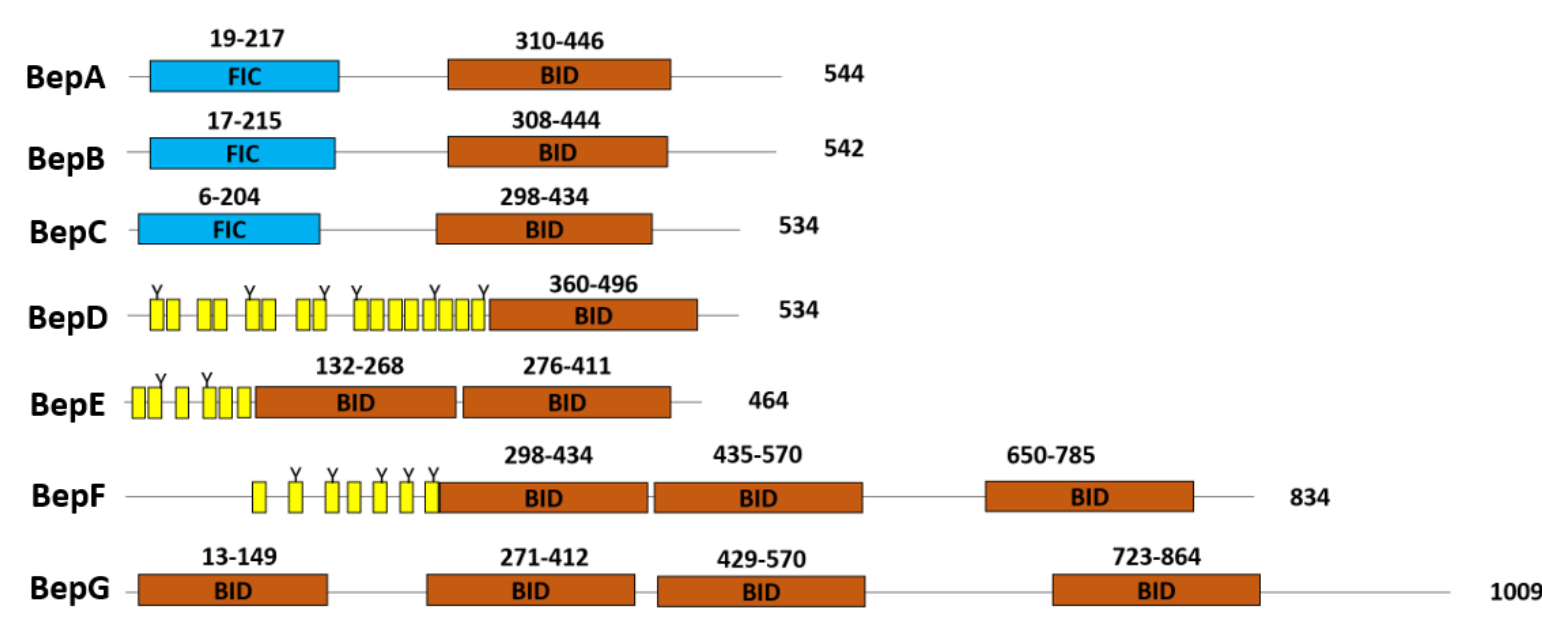

Figure 1.2: Bartonella henselae Bep protein structure. Fic domains are indicated in blue, BID domains are indicated in brown. Yellow boxes represent tyrosine-containing sequence motifs (indicated by Y). Based off figure used by Schulein et al. (2005).

While B. henselae Bep proteins are relatively well characterised, little is known about the function of the B. quintana Bep homologues. Interestingly, B. quintana also contains an gene similar to yopJ in Yersinia pestis (yopP in Y. enterocolitica) that is not found in B. henselae ${ }^{39}$. Yersinia YopJ is a Type III secreted effector protein that catalyses acetylation reactions on conserved regions in the activation loop of MAP kinase signalling proteins. This prevents activation of these signalling proteins by phosphorylation and ultimately induces macrophage cell death ${ }^{68,69}$. This suggests that YopJ interferes with the innate immune response, as it has also been shown to inhibit NF-KB signalling ${ }^{68,69}$. However, no function has been described for the B. quintana homologue.

\subsubsection{Bep Protein Functions}

The effector proteins BepA - $\mathrm{G}$ are all secreted by the VirB/D4 T4SS which together are responsible for all of the VirB/D4-dependent cellular phenotypes of $B$. henselae infected endothelial cells described above. While not all the functions of the Bep proteins are known, there have been some characterised effects. For example, either a combination of BepC and BepF, or BepG independently, are responsible for invasome formation ${ }^{3,51}$. It is believed that these two protein systems represent a form of functional redundancy, although they have been shown to act via different pathways ${ }^{51}$. These protein systems are also responsible for inhibiting endocytic uptake of bacteria to enable the invasome process, as this is the slower mechanism for bacterial internalisation. 
It is hypothesised that BepC and BepF may act at an intermediate level of the signalling cascade that leads to invasome formation ${ }^{51}$, while BepG is believed to interact further down the cascade. Interestingly, BepG also appears to have strong anti-angiogenic effects, while BepD has relatively moderate pro-angiogenic effects ${ }^{61}$. Whether this is a direct or indirect effect is not known. Recently BepE has been shown to inhibit the deleterious effects of BepC, which can cause host cell fragmentation in the absence of $\mathrm{BepE}^{40}$. BepE exerts this effect by interacting with the host cell Rho signalling pathway via its second BID domain, resulting in the stabilisation of stress fibres. Loss of BepE in Bartonella tribocorum results in a strain unable to establish bloodstream infection in a rat model using intradermal inoculation ${ }^{40}$. BepE also appears to be important in establishing colonisation of the dermal niche. The function of BepB currently remains unknown.

\subsubsection{BepA}

BepA from $B$. henselae is one of the best studied Beps and contains both a Fic domain and a BID domain ${ }^{60,66}$. The BID domain of BepA has previously been shown to inhibit apoptosis by directly binding host adenylyl cyclase in the presence of the Ga subunit to increase cAMP levels ${ }^{60,70}$. The BID domain of BepA from B. quintana also demonstrated this effect. The bepA gene in B. quintana was originally annotated as a pseudogene ${ }^{39,60}$. However, it was noted that the gene encoded an internal stop and start codon in frame splitting the gene into two open reading frames, BepA1 and BepA2. The first open reading frame encodes the Fic domain and the second encodes the BID domain and C-terminal tail ${ }^{60}$. It has not been determined whether the Fic domain of $B$. quintana BepA is also functional. Interestingly, the BID domain of Bartonella tribocorum's BepA did not demonstrate this anti-apoptotic effect. BepA from Bartonella henselae has also been shown to have a strong pro-angiogenic effect ${ }^{61}$; however, the exact function of the BepA Fic domain remains unknown. 


\subsection{The Fic Domain}

Bioinformatic analyses reveal that over 2000 species from all forms of life encode a Fic domain. The catalytic function of this domain was discovered by Yarbrough et al. (2009), who described its ability to covalently modify protein side chains with an AMP moiety, typically on a serine, threonine or tyrosine residue. This reaction is known as adenylylation or AMPylation. AMPylation was first described in 1970 as an enzymatic reaction carried out by an adenylyl transferase enzyme in $E$. coli to regulate the activity of an endogenous enzyme, glutamine synthetase $\mathrm{e}^{72,73}$.

This post translational modification is reversible, as indicated by deadenylylation of glutamine synthetase by another E. coli enzyme. Only recently has the role of AMPylation in bacterial toxicity and pathogenicity been discovered ${ }^{66,71,74-76}$.

\subsubsection{Vibrio parahaemolyticus and VopS}

One of the first proteins where Fic domain activity in pathogenicity was analysed is VopS, a protein delivered into the host cell by the Vibrio parahaemolyticus Type III secretion system ${ }^{71,74}$. When expressed in HeLa cells, this protein induced cell rounding, an indication of cytotoxicity. Yarbrough et al. (2009) demonstrated that when a constitutively activated Rac protein was co-expressed with VopS, the Rac protein mass increased by 329 daltons, consistent with addition of an AMP moiety. Further analysis revealed that an AMP moiety was added to Thr35, a highly conserved residue located in the effector loop of the switch I region of the Rac protein. VopS specificity was demonstrated by substituting Thr35 with an Ala residue, which abrogated the AMPylation reaction. Further experiments showed that VopS catalysed AMPylation reactions on other members of the Rho-GTPase family, namely Rho and Cdc42 ${ }^{71}$. The transfer of AMP results in steric hindrance and prevents activated Rho GTPase proteins from binding downstream signalling partners, interrupting signalling and leading to collapse of the actin cytoskeleton and cell rounding ${ }^{71}$. VopS has also been demonstrated to have in vitro auto-AMPylation activity ${ }^{77}$. Other Fic domain-containing proteins, such as the fruit fly protein CG9523 from Drosophila melanogaster, also have in vitro auto-AMPylation activity $^{66,77}$. 


\subsubsection{Histophilus somni and IbpA}

Another key bacterial cytotoxin, IbpA, contains two Fic domains, and is secreted by the cattle pathogen Histophilus somni ${ }^{75}$. Like VopS, this protein also catalyses an AMPylation reaction on the Rho family of GTPases, preventing binding to their downstream signalling partners ${ }^{71,75}$. When expressed in HeLa cells, IbpA caused plasma membrane retraction and actin cytoskeleton collapse, and the Fic2 domain was shown to be sufficient for this effect. The Fic2 domain of IbpA, like the Fic domain of VopS, catalyses the transfer of an AMP to the switch I region of the GTPases. However, in the case of IbpA, the AMP is transferred to a conserved Tyr residue rather than the Thr residue found in $\operatorname{Rac}^{71,75}$. The AMPylation reaction was specific for the active form of the GTPases as no activity was seen with dominant negative mutants of the GTPases.

\subsubsection{Fic Domain Motif}

For both VopS and IbpA, the function of the Fic domain is dependent on a conserved histidine residue in the Fic domain's core motif, HPFxxGNGR ${ }^{71,74,75}$. Mutants constructed with an Ala substitution for the His residue resulted in a complete loss of function as all mutants failed to induce AMPylation on their targets ${ }^{66,71,74,75}$. This core motif is conserved in $B$. henselae Bep $^{66}$ as well as BepC from both $B$. henselae and B. quintana. In the case of $B$. henselae BepB, the last two amino acids differ from the motif ( $G R \rightarrow E H$ ); in the case of $B$. quintana BepA1, the last glycine is substituted with a glutamate. However, in all instances the invariant histidine remains. This core motif is also found in death on curing (Doc) domain proteins, and it is believed that the Doc domain evolved from Fic domains ${ }^{77}$. For this reason, Fic and Doc proteins belong to the same protein superfamily, the Fido superfamily ${ }^{77}$. Interestingly, the Fic motif of IbpA showed a high sequence similarity to a human protein HYPE, which also weakly AMPylates Rho GTPases in vitro ${ }^{75}$. Recently, it was revealed that HYPE localises to the ER lumen where it plays a critical role in the unfolded protein repsonse ${ }^{78}$. HYPE acts to AMPylate the molecular chaperone BiP at Ser365 and Thr366 in the ATPase binding domain. This results in increased BiP activity for protein refolding ${ }^{78}$, and demonstrates a novel function for Fic domain-containing proteins. 


\subsubsection{Catalytic Mechanisms}

Orth et al. (2010) have obtained a crystal structure of VopS and from this have proposed a catalytic mechanism. The Fic motif residues His348, Phe350, Asp352, Asn354, and Arg356, as well as the distal Arg299, are critical for full catalytic activity. In particular, the mutations H348A and R356A had the most diminished function of any of the mutations in these critical amino acids ${ }^{79}$. As shown in Figure 1.3 below, the histidine residue acts as a base in the reaction to activate the threonine residue of the $\mathrm{Cdc} 42$ protein. This is supported by the fact that the VopS enzyme is less active at a pH below 6.0, the pKa of histidine ${ }^{79}$. Kinetic results have shown that both Asn354 and Arg356 are involved in binding the ATP substrate. Asp352 binds ATP indirectly via its bound $\mathrm{Mg}^{2+}$ ion. Finally, Arg299 stabilises the binding of the target protein, Cdc42 (not shown).

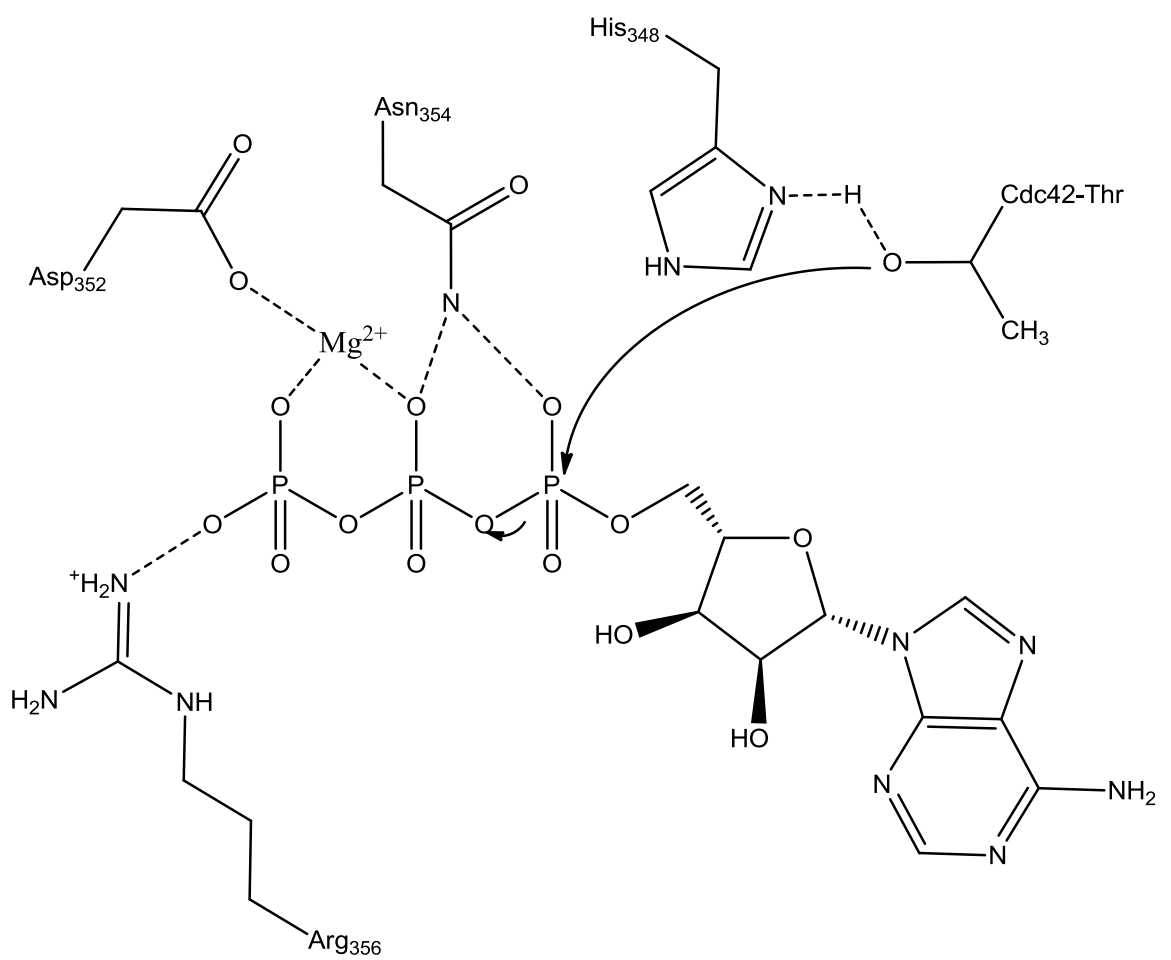

Figure 1.3: Proposed mechanism of VopS catalysed AMPylation of Cdc42. The diagram shows the stabilisation of the phosphate groups by the VopS active site amino acids. The Cdc42 threonine is activated by the critical histidine in the VopS active site resulting in nucleophilic attack on the primary phosphate group. Diagram based off catalytic mechanism illustrated by Orth et al. (2010)

Similarly, Dixon et al. (2010) have published the crystal structure for the IbpA Fic2 domain bound to $\mathrm{Cdc} 42$. They propose a similar catalytic mechanism to that illustrated in Figure 1.3. The invariant histidine residue is thought to act as a base and interact with the proton of the Cdc42 tyrosine hydroxyl group ${ }^{80}$. This interaction increases the nucleophilicity of the tyrosine hydroxyl group, which attacks the $\alpha$-phosphate of the bound ATP (Figure 1.4). 


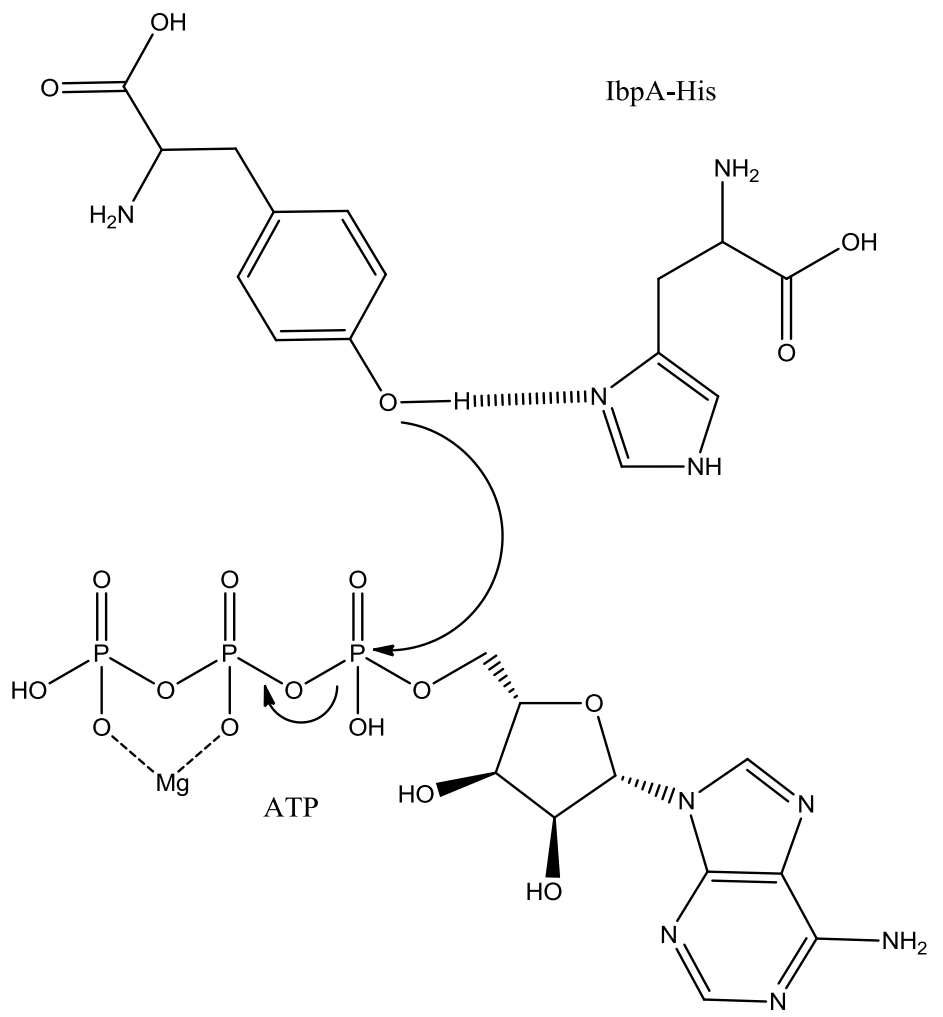

Figure 1.4: Proposed mechanism of IbpAFic2 catalysed AMPylation of Cdc42. The diagram shows the activation of the Cdc42 tyrosine by the critical histidine in the IbpA-Fic2 activation site. This results in nucleophilic attack on the $\alpha$-phosphate by the activated tyrosine. Diagram based off catalytic mechanism illustrated by Dixon et al. (2010)

\subsubsection{Toxin/Antitoxin Modules}

Fic domain-containing proteins have also been identified in toxin/anti-toxin modules, which play a role in maintenance of bacterial plasmids ${ }^{81}$. Toxin/anti-toxin systems include a stable protein (toxin), which exerts a toxic effect on the plasmid-containing cell, while another, less stable molecule (protein or non-coding RNA) inhibits the activity of the toxin ${ }^{81,82}$. In many cases, toxin/antitoxin modules are found on plasmids and effectively kill bacteria that lose the plasmid (losing the less stable anti-toxin, but retaining the stable toxin). However, these modules are also found on bacterial chromosomes ${ }^{82}$. Bartonella schoenbuchensis, a Group A species, expresses VbhT, a protein with both a Fic domain and a BID domain, like several Bartonella effectors ${ }^{15,23}$. While it is unknown if VbhT has a mammalian target cell or function, it arrests growth in E. coli when expressed ${ }^{23}$. Like the other Fic domain proteins described above, it contains the conserved motif and invariant histidine residue and catalyses an AMPylation reaction on an unknown $80 \mathrm{kDa}$ E. coli protein ${ }^{23}$. 
Interestingly, co-expression with VbhA, a protein encoded upstream of $\mathrm{VbhT}$, completely represses $\mathrm{VbhT}$ toxicity ${ }^{23}$. The VbhA protein was shown to bind to the ATP binding site of VbhT and block the AMPylation reaction ${ }^{23}$. These two proteins represent an example of a Fic toxinantitoxin module ${ }^{23}$.

\subsubsection{Other Fic domain activities}

It should be noted that not all Fic proteins AMPylate their target substrates. A study of the Legionella pneumophila Fic protein Ank showed that it transferred a phosphocholine moiety to its target proteins, in this case the Rab GTPases ${ }^{76}$. Intriguingly, the L. pneumophila Type IV secreted protein Drr lacks a Fic domain, yet AMPylates RabB1-GTPase ${ }^{76}$.

Recently, the activity of a related Bartonella Fic-containing protein was described. Using a mass spectrometry approach, the Fic domain of Bep2 from B. rochalimae, a recently discovered Group B Bartonella ${ }^{83}$, was found to AMPylate vimentin, a type III intermediate filament of mesenchymal derived cells ${ }^{84,85}$. This illustrates the range of diverse activities, even among proteins with conserved domains.

\subsubsection{Role of Bartonella henselae BepA Fic Domain}

$B$. henselae BepA is the best studied Bep protein and is responsible for inhibiting apoptosis through its BID domain. BepA also contains a Fic domain which Palanivelu et al. (2011) have heterologously expressed and proteolytically cleaved to yield a fragment containing this domain. This fragment displayed auto-AMPylation activity, and also AMPylated two unknown HeLa cell proteins of around 40 and $50 \mathrm{kDa}^{66}$. These masses do not correspond with any of the Rho-GTPase family and likely represent two uncharacterised AMPylation targets ${ }^{66}$. The AMPylation reaction was also dependent on the conserved Fic domain histidine residue. Interestingly, Palanivelu et al. (2011) found that the reaction was not strictly dependent on ATP, as GMP and CMP moieties were incorporated with almost equal efficiency when incubated with GTP and CTP respectively ${ }^{66}$. 


\subsection{Research Aims}

The aim of this research was to identify the eukaryotic host cell target protein(s) of the Fic domains of $\mathrm{BepA}, \mathrm{Bep} \mathrm{B}$, and $\mathrm{BepC}$, from $B$. henselae, and BepA1 and $\mathrm{BepC}$, from B. quintana. To do this, portions of the bep genes, containing the Fic domain but excluding the BID domain, were cloned into an expression plasmid and the Fic-containing protein purified using nickel affinity chromatography. Whole cell extracts from human endothelial and epithelioid cells were incubated with one of two labelled ATP analogues and the purified Fic domain peptide. The resulting reactions were run on SDS-PAGE gels, with the aim of identifying target proteins by fluorescence. The goal of this work was to detect and identify the host cell protein(s) modified by the transfer of the ATP analogue by the Bartonella Fic domains. Candidate protein(s) would then be excised from the gel and analysed by mass spectrometry to identify the targeted proteins, modified by the transfer of the ATP analogues by the recombinant Bep proteins.

Recently vimentin was identified as a host cell target protein of Bep2 from B. rochalimae, a related species to $B$. henselae and $B$. quintana. Vimentin is a cytoskeletal protein found in endothelial cells, and could represent a potential target of $B$. henselae and B. quintana Beps. To this end, vimentin polymerisation assays were also performed along with mass spectrometry analysis to determine if the recombinant Bep proteins were able to modify vimentin, and if this modification caused interference in the polymerisation of vimentin monomers. 


\section{General Methods}

\subsection{Media Recipes}

All media were autoclaved at $121^{\circ} \mathrm{C}$ for at least 15 minutes, and cooled until supplements could be added.

\subsubsection{Liquid Media}

Lysogeny Broth (LB)

- $1 \%$ Tryptone

- $0.5 \%$ Yeast Extract

- $0.5 \% \mathrm{NaCl}$

$\underline{2 X \text { YT Media }}$

- $1.6 \%$ Tryptone

- $1.0 \%$ Yeast Extract

- $0.5 \% \mathrm{NaCl}$

$\underline{\text { SOC Media }}$

- $2 \%$ Tryptone

- $0.5 \%$ Yeast Extract

- $0.05 \% \mathrm{NaCl}$

- $1 \% 250 \mathrm{mM} \mathrm{KCl}$

Autoclaved then $0.5 \% 2 \mathrm{M}$ sterile $\mathrm{MgCl}_{2}$ and $2 \% 1 \mathrm{M}$ sterile glucose added.

\subsubsection{Solid Media}

\section{Chocolate Agar}

Solutions were boiled before being autoclaved separately and then combined

- $500 \mathrm{~mL}$ 2\% BBL Freeze-dried Bovine Haemoglobin solution (Fort Richard Labs)

- $500 \mathrm{~mL}$ 7.2\% GC agar (Thermo Fisher Scientific) solution

- $1 \%$ IsoVitaleX Enrichment Medium (Becton Dickinson) added once autoclaved solution had cooled 


\section{$\underline{\text { LB agar }}$}

LB agar was made up by adding $1.5 \%$ agar to the LB solution described above.

\subsubsection{Supplement Solutions}

\begin{tabular}{|l|l|}
\hline Solution & Final Concentration \\
\hline Kanamycin Sulfate & $50 \mu \mathrm{g} / \mathrm{mL}$ \\
\hline Ampicillin & $100 \mu \mathrm{g} / \mathrm{mL}$ \\
\hline Isopropyl-D-1-thiogalactopyranoside (IPTG) & $1 \mathrm{mM}$ \\
\hline Glucose & $20 \mathrm{mM}$ \\
\hline
\end{tabular}

Table 2.1: Supplements added to media.

\subsection{Bacterial Strains and Growth Conditions:}

\subsubsection{Bacterial Strains}

\begin{tabular}{|l|l|l|}
\hline $\begin{array}{l}\text { Strain } \\
\begin{array}{l}\text { Bartonella henselae } \\
\text { Houston-1 Strain }\end{array}\end{array}$ & $\begin{array}{l}\text { Characteristics } \\
\text { male }\end{array}$ & Source \\
\hline $\begin{array}{l}\text { Bartonella quintana JK-31 } \\
\text { Strain }\end{array}$ & $\begin{array}{l}\text { Isolated from a cutaneous bacillary } \\
\text { angiomatosis lesion of a human patient }\end{array}$ & Dr Jane Koehler ${ }^{54}$ \\
\hline E. coli DH5 $\alpha$ & $\begin{array}{l}\text { recA1 and endA1 mutations, blue/white } \\
\text { screening }\end{array}$ & Life Technologies \\
\hline E. coli BL21 & Deficient in Lon and OmpT Proteases & Lab Stock \\
\hline E. coli TOP10 & $\begin{array}{l}\text { endA1, and recA1 mutations, hsdR, mcrA, } \\
\text { lacZDM15 for blue/white screening }\end{array}$ & Thermo Scientific \\
\hline
\end{tabular}

Table 2.2: Bacterial strains used in this study.

\subsubsection{Bartonella Growth Conditions}

Bartonella henselae Houston-1 strain $^{8}$ was cultured on chocolate agar plates under an enriched $\mathrm{CO}_{2}$ atmosphere, using GenBags (Biomérieux) to establish capnophilic conditions. The bacteria were incubated at $37^{\circ} \mathrm{C}$ for $5-7$ days. 


\subsubsection{E. coli Growth and Storage Conditions}

All E. coli were grown overnight at $37^{\circ} \mathrm{C}$, either in $5 \mathrm{~mL}$ LB liquid media, shaking at $200 \mathrm{rpm}$, or by plating bacterial suspension $(100 \mu \mathrm{L})$ on LB agar plates. Where necessary, the media was supplemented with antibiotic. Glycerol stocks were preserved with $30 \%$ glycerol in cryovials and stored at $-80^{\circ} \mathrm{C}$.

\subsection{Plasmid Transformation of Competent Cells}

\subsubsection{Preparing Electrocompetent Cells}

$\mathrm{DH} 5 \alpha$ or BL21 E. coli were cultured overnight in LB media. Overnight cultures were inoculated into two autoclaved, pre-warmed conical flasks containing $250 \mathrm{~mL} L B$ at a starting $\mathrm{OD}_{600}$ of 0.1. Flasks were incubated at $37^{\circ} \mathrm{C}$ with shaking; until the $\mathrm{OD}_{600}$ reached $0.3-0.5$. Cultures were cooled in an ice cold water bath and swirled occasionally for 30 minutes, then transferred to ice cold centrifuge bottles and spun for 15 minutes at $1000 \times g$ at $4-10^{\circ} \mathrm{C}$. The supernatant was discarded and the pellets were washed three more times with the following, once each:

1. Ice cold sterile $\mathrm{ddH}_{2} \mathrm{O}(250 \mathrm{~mL})$

2. Ice cold glycerol $(10 \%, 125 \mathrm{~mL})$

3. Ice cold glycerol $(10 \%, 5 \mathrm{~mL})$

Each wash was spun for 20 minutes at $1000 \times g$ at $4-10^{\circ} \mathrm{C}$. Cells were re-suspended in $1 \mathrm{~mL}$ of $10 \%$ ice cold glycerol and distributed in $40 \mu \mathrm{L}$ aliquots to cryovials which were frozen at $-80^{\circ} \mathrm{C}$ until needed.

\subsubsection{Electroporation}

Electrocompetent $E$. coli cells were defrosted on ice before addition of a purified ligation mixture of plasmid $(5 \mu \mathrm{L})$, then incubated on ice for 30 seconds. The suspension was transferred to a dry, ice cold $0.2 \mathrm{~cm}$ electroporation cuvette. The bacterial suspension was electroporated (Biorad Gene Pulser Xcell) using standard settings for E. coli ( $25 \mu \mathrm{F}, 2.5 \mathrm{kV}, 200$ $\Omega)$. The cells were immediately placed in $1 \mathrm{~mL}$ of warm $2 \mathrm{X}$ YT or SOC media and incubated for 1 hour at $37^{\circ} \mathrm{C}$ and $200 \mathrm{rpm}$. After recovery, the transformed cells were plated onto selective media at normal and 1:10 dilutions $(100 \mu \mathrm{L})$, with the remainder being pelleted and resuspended in $100 \mu \mathrm{L}$ LB before plating. Resulting cultures were incubated overnight at $37^{\circ} \mathrm{C}$. 


\subsubsection{Transforming Chemically Competent Cells}

TA cloning solution $(2 \mu \mathrm{L}$ ) was added to a vial of TOP10 chemically competent cells (Thermo Scientific) and mixed by flicking the tube. Cells were then incubated on ice for 10 minutes before being heat shocked at $42^{\circ} \mathrm{C}$ for 30 seconds. The cells were transferred back to ice and SOC medium ( $250 \mu \mathrm{L}$ ) was added. The cells were incubated at $37^{\circ} \mathrm{C}$ and $200 \mathrm{rpm}$ for 1 hour.

Cells were then plated at varying concentrations on LB plates containing $100 \mu \mathrm{g} / \mathrm{mL}$ ampicillin. Plates were incubated overnight at $37^{\circ} \mathrm{C}$.

\subsection{Standard Molecular Biology Techniques}

\subsubsection{Primers}

All primers used in this study were synthesised by Integrated DNA Technologies (IDT, Singapore) and supplied lyophilised. For long term storage, primers were suspended in sterile $\mathrm{ddH}_{2} \mathrm{O}$ to a final concertation of $100 \mu \mathrm{M}$. For working stocks, aliquots were suspended in sterile $\mathrm{ddH}_{2} \mathrm{O}$ to $10 \mu \mathrm{M}$ final concentration. All primers were stored at $-20^{\circ} \mathrm{C}$.

Forward primers were designed so that the ATG start site for each gene was included in the Ndel restriction site, enabling an in-frame insertion of the open reading frame gene downstream of an $\mathrm{N}$-terminal $\mathrm{His}_{6}$-tag in the $\mathrm{pET} 28 \mathrm{a}$ vector. B. quintana BepC was the exception, as this gene contained an internal Ndel restriction site. In this case an Nhel site was engineered in the primer before the start of the gene. The reverse primers were designed to contain an Xhol restriction site immediately following the gene. These primers also contained an engineered stop codon to prevent addition of a C-terminal His-tag. 


\subsubsection{Primers for Bacterial Expression}

\begin{tabular}{|l|l|}
\hline Primer Names & $\underline{\text { Primer Sequence }}$ \\
\hline BepFicNdel_For & 5'- GCG CAT ATG CCA AAG GCA AAA GCA AAA ACG -3' \\
\hline BepFicXhol_Rev2 & 5'- GCG CTC GAG TCA GGC TTT TGG AGC TGT AAA GGT -3' \\
\hline BhBepBFicNdel_For & 5'- GCG CAT ATG CCA AAA GCA AAA GCA -3' \\
\hline BhBepBFicXhol_Rev & 5'- GCG CTC GAG TCA GGC GAT AAA GGT AAT TGT -3' \\
\hline BhBepCFicNdel_For2 & 5'- GCG CAT ATG TTA GAG CAT AAT TAT CTG TAT AAA AAC AGC -3' \\
\hline BhBepCFicXhol_Rev2 & 5'- GCG CTC GAG TCA GAC TAT GCG ATC ACC GTT CTG -3' \\
\hline BqBepAFic_NdelF & 5' - GCG CAT ATG CCA AAA GCA AAG TCA AAA GAA ACG -3' \\
\hline BqBepAFic_XholR & 5'- GCG CTC GAG TCA AAC AGA TGT TTC ATA GGC TCT AGA TTG -3' \\
\hline BqBepCFic_NheIF & 5'- GCG GCT AGC ATG TTA GAG CAT AAT TAT TTT TAT AAA AAC AGC GCA \\
\hline -3' \\
\hline BqBepCFic_XholR & 5'- GCG CTC GAG TCA CTG AAA GGA GAT GAA ATC ACC ATT CTG -3' \\
\hline BhBepA_H159A_F & 5'- CAA CCA ACT CGC CCC ATT CAG AGA AGG GAA TGG ACG -3' \\
\hline BhBepA_H159A_R & 5'- TTC TCT GAA TGG GGC GAG TTG GTT GAG AGA ATT AAA C -3' \\
\hline T7 Promoter & 5'- TAA TAC GAC TCA CTA TAG GG -3' \\
\hline T7 Terminator & 5'- GCT AGT TAT TGC TCA GCG G -3' \\
\hline
\end{tabular}

Table 2.3: Primers used for cloning into E. coli. Bold shows the restriction site, underline shows an engineered stop codon.

\subsubsection{Primers for Expression in Mammalian Cells}

\begin{tabular}{|l|l|}
\hline Primer Names & Primer Sequence \\
\hline mCT-GFP-BhBepA_F & 5' - ACC ATG GGG ATG CCA AAG GCA AAA GCA AAA ACG - 3' \\
\hline mCT-GFP- BhBepA_R & 5' - G GGC TTT TGG AGC TGT AAA GGT AAT TTT ATC ACC - 3' \\
\hline GFP Reverse & 5'- TAG AAG GCA CAG TCG AGC -3' \\
\hline
\end{tabular}

Table 2.4: Primers used for cloning mammalian cell expression plasmids. Bold indicates the engineered Kozak Sequence. 


\subsubsection{Plasmids}

\subsubsection{Bacterial Plasmids}

\begin{tabular}{|c|c|c|c|}
\hline Strain & Properties & Primers & Source \\
\hline pET28a (Novagen) & $\begin{array}{l}\text { Bacterial Expression vector, His- } \\
\text { Tag }\end{array}$ & N/A & $\begin{array}{l}\text { Merck } \\
\text { Millipore }\end{array}$ \\
\hline pBhBepAFic_37 & $\begin{array}{l}\text { Derivative of pET28a encoding } B h \\
\text { BepA fragment (aa } 1-303 \text { ) }\end{array}$ & $\begin{array}{l}\text { BepFicNdel_For } \\
\text { BepFicXhol_Rev2 }\end{array}$ & This Study \\
\hline pBhBepBFic_19 & $\begin{array}{l}\text { Derivative of pET28a encoding } B h \\
\text { BepB fragment (aa } 1-298)\end{array}$ & $\begin{array}{l}\text { BhBepBFicNdel_For } \\
\text { BhBepBFicXhol_Rev }\end{array}$ & This Study \\
\hline pBhBepCFic_10 & $\begin{array}{l}\text { Derivative of pET28a encoding } B h \\
\text { BepC fragment (aa } 1-285 \text { ) }\end{array}$ & $\begin{array}{l}\text { BhBepCFicNdel_For } \\
\text { BhBepCFicXhol_Rev }\end{array}$ & This Study \\
\hline pBqBepA1_1 & $\begin{array}{l}\text { Derivative of pET28a encoding } B q \\
\text { BepA1 }\end{array}$ & $\begin{array}{l}\text { BqBepAFic_NdelF } \\
\text { BqBepAFic_XholR }\end{array}$ & This Study \\
\hline pBqBepCFic_2 & $\begin{array}{l}\text { Derivative of pET28a encoding } B q \\
\text { BepC fragment (aa } 1-289 \text { ) }\end{array}$ & $\begin{array}{l}\text { BqBepCFic_NhelF } \\
\text { BqBepCFic_XholR }\end{array}$ & This Study \\
\hline pBhBepAFicH159A_40 & $\begin{array}{l}\text { Derivative of pET28a encoding a } \\
\text { mutated (H ->A) Bh BepA (aa } 1 \text { - } \\
\text { 303) }\end{array}$ & $\begin{array}{l}\text { BepFicNdel_For } \\
\text { BepFicXhol_Rev2 } \\
\text { BhBepA_H159A_F } \\
\text { BhBepA_H159A_R }\end{array}$ & This Study \\
\hline pBrBep2Fic_5 & $\begin{array}{l}\text { Derivative of pET28a encoding } B r \\
\text { Bep2 fragment (aa } 1-360 \text { ) }\end{array}$ & $\begin{array}{l}\text { VopS_pET28a } \\
\text { (gBlock) }\end{array}$ & This Study \\
\hline pVpVopS_10 & $\begin{array}{l}\text { Derivative of } p E T 28 a \text { encoding } V p \\
\text { Serotype } 04: K 12 \text { VopS }\end{array}$ & $\begin{array}{l}\text { Bep2_pET28a } \\
\text { (gBlock) }\end{array}$ & This Study \\
\hline
\end{tabular}

Table 2.5: Bacterial plasmids generated in this study.

2.4.2.2 Plasmids for Mammalian Expression

\begin{tabular}{|l|l|l|l|}
\hline Strain & Properties & Primers & $\underline{\text { Source }}$ \\
\hline pBhBepAFicGFP_14 & $\begin{array}{l}\text { Derivative of pcDNA3.1-CT- } \\
\text { GFP-TOPO (source) } \\
\text { containing BhBepAFic }\end{array}$ & $\begin{array}{l}\text { mCT-GFP-BhBepA_F } \\
\text { mCT-GFP-BhBepA_R }\end{array}$ & This Study \\
\hline pBhBepAFicH159AGFP_10 & $\begin{array}{l}\text { Derivative of pcDNA3.1-CT- } \\
\text { GFP-TOPO (source) } \\
\text { containing a mutated } \\
\text { BhBepAFic }\end{array}$ & $\begin{array}{l}\text { mCT-GFP-BhBepA_F } \\
\text { mCT-GFP-BhBepA_R } \\
\text { BhBepA_H159A_F } \\
\text { BhBepA_H159A_R }\end{array}$ & This Study \\
\hline
\end{tabular}

Table 2.6: Eukaryotic plasmids generated in this study. 


\subsubsection{Isolation of Plasmid DNA}

DNA minipreps were performed using the Presto Plasmid Miniprep kit (Geneaid) as per the manufacturer's instructions. Plasmid DNA was eluted in $50 \mu \mathrm{L}$ of elution buffer (10 mM Tris$\mathrm{HCl}, \mathrm{pH} 8.5)$.

\subsubsection{Isolation of Genomic DNA}

Genomic DNA was isolated using a modified version of the CTAB extraction method ${ }^{86}$. Briefly B. henselae from 5-7 day old chocolate agar plates was scraped off and re-suspended in 567 $\mu \mathrm{L}$ of TE buffer (10 mM Tris, $1 \mathrm{mM}$ EDTA). Re-suspended bacteria were lysed with $30 \mu \mathrm{L}$ of $10 \%$ SDS and proteins removed with $3 \mu \mathrm{L}$ of $20 \mathrm{mg} / \mathrm{mL}$ proteinase $\mathrm{K}$, followed by a 1 hour incubation at $37^{\circ} \mathrm{C}$. To precipitate protein and lipid breakdown products, the solution was treated with $100 \mu \mathrm{L}$ of $5 \mathrm{M} \mathrm{NaCl}$ followed by $80 \mu \mathrm{L}$ of CTAB/NaCl solution ( $10 \% \mathrm{CTAB}$ in $0.7 \mathrm{M}$ $\mathrm{NaCl})$ and incubated at $65^{\circ} \mathrm{C}$ for 10 minutes. Genomic DNA was purified with a chloroform: isoamyl alcohol (24:1) extraction, followed by a phenol: chloroform: isoamyl alcohol (25:24:1) extraction. DNA was precipitated with 0.6 volumes of isopropanol and washed with $70 \%$ ethanol solution. Finally, DNA was re-dissolved in $100 \mu \mathrm{L}$ TE buffer.

B. quintana strain JK-31 genomic DNA was a gift from Dr Jane Koehler.

\subsubsection{Polymerase Chain Reactions}

\subsubsection{Cloning PCR}

PCR cloning reactions were performed using the Advantage polymerase (Clontech) with the following reaction components:

\begin{tabular}{|l|l|l|}
\hline$\underline{\text { Reagents }}$ & Stock Concentration & Final Concentration \\
\hline Advantage 2 PCR Buffer & $10 \mathrm{X}$ & $1 \mathrm{X}$ \\
\hline DNA Template & $100 \mathrm{ng} / \mu \mathrm{L}$ & $2 \mathrm{ng} / \mu \mathrm{L}$ \\
\hline Forward primer & $10 \mu \mathrm{M}$ & $0.2 \mu \mathrm{M}$ \\
\hline Reverse primer & $10 \mu \mathrm{M}$ & $0.2 \mu \mathrm{M}$ \\
\hline dNTP mix (Biolabs) & $10 \mathrm{mM}$ each & $200 \mu \mathrm{M}$ each \\
\hline Advantage 2 Polymerase Mix & $50 \mathrm{X}$ & $1 \mathrm{X}$ \\
\hline
\end{tabular}

Table 2.7: Reaction components for PCR with Advantage Polymerase.

Standard PCR reaction conditions are outlined below: 


\begin{tabular}{|l|l|l|l|}
\hline Pre-Cycle & $95^{\circ} \mathrm{C}$ & 1 minute & \\
\cline { 1 - 3 } Denaturing Step & $95^{\circ} \mathrm{C}$ & 30 seconds & \multirow{3}{*}{30 Cycles } \\
\cline { 1 - 2 } Annealing Step & $60^{\circ} \mathrm{C}$ & 30 seconds & \\
\cline { 1 - 3 } Extension Step & $68^{\circ} \mathrm{C}$ & 1 minute & \\
\hline Post-Cycle & $68^{\circ} \mathrm{C}$ & 1 minute & \\
\hline
\end{tabular}

Table 2.8: Reaction conditions used for Advantage PCR with Bep-Fic primers.

For TA cloning into the pcDNA3.1 mammalian expression vector, the post-cycle extension step was lengthened to 10 minutes. Interestingly, for both bepC genes, the Advantage kit did not result in product, so the Phusion Hi-Fidelity PCR kit (Thermo Scientific) was used with the following reaction conditions:

\begin{tabular}{|l|l|l|}
\hline$\underline{\text { Reagents }}$ & Stock Concentration & Final Concentration \\
\hline $5 X$ GC Buffer PCR Buffer & $5 \mathrm{X}$ & $1 \mathrm{X}$ \\
\hline DNA Template & $100 \mathrm{ng} / \mu \mathrm{L}$ & $2 \mathrm{ng} / \mu \mathrm{L}$ \\
\hline Forward primer & $10 \mu \mathrm{M}$ & $0.2 \mu \mathrm{M}$ \\
\hline Reverse primer & $10 \mu \mathrm{M}$ & $0.2 \mu \mathrm{M}$ \\
\hline dNTP mix (Biolabs) & $10 \mathrm{mM}$ each & $200 \mu \mathrm{M}$ each \\
\hline Phusion Polymerase Mix & $50 \mathrm{X}$ & $1 \mathrm{X}$ \\
\hline
\end{tabular}

Table 2.9: Reaction components for PCR with Phusion Polymerase.

\begin{tabular}{|l|l|l|l|}
\hline Pre-Cycle & $98^{\circ} \mathrm{C}$ & 30 seconds & \\
\cline { 1 - 2 } Denaturing Step & $98^{\circ} \mathrm{C}$ & 10 seconds & \multirow{3}{*}{30 Cycles } \\
\cline { 1 - 2 } & $60^{\circ} \mathrm{C}$ & 30 seconds & \\
\cline { 1 - 3 } Extension Step & $72^{\circ} \mathrm{C}$ & 30 seconds & \\
\hline Post-Cycle & $72^{\circ} \mathrm{C}$ & 5 minutes & \\
\hline
\end{tabular}

Table 2.10: Reaction conditions used for Phusion PCR for BepC-Fic constructs.

Control reactions with no added template DNA were routinely run. 


\subsubsection{BepA_H159A mutant}

In order to construct the BhBepA_H159A mutant, a PCR fusion site-directed mutagenesis strategy was employed. Overlapping primers were engineered to contain two base changes converting the histidine in the active site into an alanine. A two-part PCR reaction was carried out; in the first step two overlapping fragments with the engineered mutation were amplified by PCR (Reactions 1 and 2). These PCR products were purified and checked, then used as a template in Reaction 3, which fused and amplified these two products, incorporating the mutation. The primers used for Reactions 1-3 are listed in Table 2.11.

\begin{tabular}{|l|l|l|l|}
\hline & Reaction 1 & $\underline{\text { Reaction 2 }}$ & $\underline{\text { Reaction 3 }}$ \\
\hline Forward Primer & BhBepA_H159A_F & BepFicNdel_For & BepFicNdel_For \\
\hline Reverse Primer & BepFicXhol_Rev2 & BhBepA_H159A_R & BepFicXhol_Rev2 \\
\hline
\end{tabular}

Table 2.11 Site directed mutagenesis for BhBepA_H159A-Fic construction. The table indicates the primers used for each PCR reaction. For Reactions 1 and 2, B. henselae genomic DNA was used as the template. For Reaction 3, the PCR products from Reactions 1 and 2 were combined and used as a template. Primer sequences are described in Table 2.3.

All reactions were performed using the Advantage PCR conditions described in Table 2.7, however the extension length was increased to 3 minutes. For the pcDNA3.1 mammalian expression vector, the mCT-GFP-BhBepA primers were used in place of the BepFic primers (Table 2.4)

\subsubsection{Colony PCR}

To screen colonies for plasmids with the correct insertion, colonies on solid media were added to premixed PCR reagents with a sterile inoculating loop. Colony PCR was carried out using Biomix Red Polymerase Mastermix (Bioline) with the following reaction components in a 10 $\mu \mathrm{L}$ total volume:

\begin{tabular}{|l|l|l|}
\hline Reagents & Stock Concentration & Final Concentration \\
\hline $2 \mathrm{X}$ Biomix Red & $2 \mathrm{X}$ & $1 \mathrm{X}$ \\
\hline Forward primer & $10 \mu \mathrm{M}$ & $0.5 \mu \mathrm{M}$ \\
\hline Reverse primer & $10 \mu \mathrm{M}$ & $0.5 \mu \mathrm{M}$ \\
\hline
\end{tabular}

Table 2.12: Reaction components for colony PCR. 
A positive control using $1 \mu \mathrm{L}$ plasmid DNA, and a negative control with no added DNA, were also run. The PCR reactions were carried out under the following conditions:

\begin{tabular}{|l|l|l|l|}
\hline Pre-Cycle & $95^{\circ} \mathrm{C}$ & 10 minutes & \\
\hline Denaturing Step & $95^{\circ} \mathrm{C}$ & 30 seconds & \multirow{3}{*}{30 Cycles } \\
\hline Annealing Step & $45^{\circ} \mathrm{C}$ & 30 seconds & \\
\hline Extension Step & $72^{\circ} \mathrm{C}$ & 45 seconds & \\
\hline Post-Cycle & $72^{\circ} \mathrm{C}$ & 5 minutes & \\
\hline
\end{tabular}

Table 2.13: Reaction conditions for colony PCR using T7-primers.

\subsection{5 gBlocks}

Positive control proteins, V. parahaemolyticus (serotype O4:K12) VopS (GenBank: KEE64915.1 ${ }^{87}$ ) and B. rochalimae Bep2 (NCBI WP_035006139) were synthesised by IDT. In the case of VopS, Nhel and Xhol restriction sites were included, while for Bep2, BamHI and Xhol restriction sites were included. To resuspend the gBlocks, tubes were briefly centrifuged, then TE buffer was added to reach a final concentration of $10 \mathrm{ng} / \mu \mathrm{L}$. The gBlocks were subsequently vortexed and incubated at $55^{\circ} \mathrm{C}$ for 20 minutes.

Before cloning reactions were performed, gBlock DNA (35 $\mu \mathrm{L})$ was cleaned and concentrated on a Zymo column (Zymo Research), before eluting in $10 \mu \mathrm{LddH}{ }_{2} \mathrm{O}$, to remove any potentially interfering EDTA.

\subsubsection{DNA Modification Reactions}

\subsubsection{Restriction Enzyme Reactions}

Purified PCR products and plasmids were digested with Nde-I and Xho-I (20 U; New England Biolabs) at $37^{\circ} \mathrm{C}$ for 1 hour in $1 \mathrm{X}$ CutSmart Buffer (20 $\mu \mathrm{L}$ final volume), followed by heat inactivation of the enzymes at $65^{\circ} \mathrm{C}$ for 20 minutes. For $B$. quintana bepC, the PCR product and PET28a plasmid were first digested with Xho-I as above, before purifying on a Zymo column (Zymo Research). Purified PCR product and plasmid was subsequently digested with Nhe-I (10 U; Fermentas) at $37^{\circ} \mathrm{C}$ for 1 hour in $1 X$ Tango Buffer, followed by heat inactivation of the enzyme at $65^{\circ} \mathrm{C}$ for 20 minutes. For bep2, the gBlock DNA and the pET28a plasmid were digested with BamH-I and Xho-I (10 U; Thermo Scientific) in 1X Fast Digest Buffer for 1 hour at $37^{\circ} \mathrm{C}$, followed by heat inactivation at $65^{\circ} \mathrm{C}$ for 20 minutes. 


\subsubsection{Ligation Reactions}

Ligations were performed overnight at $16^{\circ} \mathrm{C}$ on all purified restriction digest samples $(8 \mu \mathrm{L})$ using T4 DNA ligase (5 $\mathrm{U}$ ) in 1X Ligation Buffer (New England Biolabs), in a final volume of 10 $\mu \mathrm{L}$. Ligation reactions were dialysed by drop dialysis with $\mathrm{dd}_{2} \mathrm{O}$ for an hour on $0.025 \mu \mathrm{m}$ membrane filters (Millipore). Resulting ligations $(5 \mu \mathrm{L})$ were used for electroporation.

\subsubsection{TA Cloning}

To clone PCR products into the pcDNA3.1/CT-GFP-TOPO ${ }^{\circledR}$ mammalian expression vector (Life Technologies), the following reaction was set up:

\begin{tabular}{|l|l|}
\hline Reagents & Volume Used \\
\hline PCR Product & $4 \mu \mathrm{L}$ \\
\hline Salt Solution $\left(1.2 \mathrm{M} \mathrm{NaCl}, 0.06 \mathrm{M} \mathrm{MgCl}_{2}\right)$ & $1 \mu \mathrm{L}$ \\
\hline pcDNA3.1 vector & $1 \mu \mathrm{L}$ \\
\hline
\end{tabular}

Table 2.14: Reaction components for pcDNA3.1 TA cloning.

The reaction was gently mixed then incubated at ambient temperature for 30 minutes. Resulting solutions $(2 \mu \mathrm{L})$ were used to transform TOP10 chemically competent cells.

\subsubsection{DNA Purification}

PCR products and restriction digests were purified using the GeneHlow Gel/PCR purification kit (Geneaid). The products were eluted in $20-50 \mu \mathrm{L}$ Elution Buffer (10 mM Tris- $\mathrm{HCl}, \mathrm{pH} 8.5$ ) or heated $\mathrm{dd}_{2} \mathrm{O}$, as required.

\subsubsection{DNA Quantification}

The quantity and purity of DNA samples was determined using a NanoDrop ND-1000 TM spectrophotometer (Thermo Scientific).

\subsubsection{TAE Agarose Gels (1\%)}

DNA samples were analysed on agarose gels ( $1 \% \mathrm{w} / \mathrm{v}$ agarose in $1 \mathrm{X}$ TAE buffer $[40 \mathrm{mM}$ Trisacetate, $1 \mathrm{mM}$ EDTA]), supplemented with $1 \mathrm{X}$ GelRed $^{\mathrm{TM}}$ (Biotium), covered with $1 \mathrm{XTAE}$ at 100 $V$ for 60 minutes. DNA was visualised under UV light. DNA loading dye (Thermo Scientific) was added to samples, which were run with 2-log DNA ladder (New England Biolabs) or Hyperladder (Bioline) as DNA size standards. 


\subsubsection{Sequencing}

All sequencing reactions were performed by Macrogen Inc (Seoul, South Korea). Sequence data was confirmed using the Geneious 8.1.7 Multiple Sequence Alignment function.

\subsection{Protein Manipulation Protocols}

\subsubsection{Inducing cellular protein}

\subsubsection{Preliminary Induction}

Overnight cultures of plasmid-containing BL21 cells were grown at $37^{\circ} \mathrm{C}, 200 \mathrm{rpm}$, and used to inoculate $5 \mathrm{~mL}$ of fresh kanamycin supplemented LB at a starting $\mathrm{OD}_{600}$ of 0.1 the following morning. Cells were returned to the incubator and after 2 hours of growth, $1 \mathrm{~mL}$ of the culture was removed pelleted by centrifugation at $16,100 \times g$ for 1 minute and set aside (un-induced control). Recombinant protein production was induced for 4 hours at $37^{\circ} \mathrm{C}$ by the addition of IPTG. After the induction, $1 \mathrm{~mL}$ of culture was removed to a fresh tube and pelleted by centrifugation, with the pellet being retained. Pellets were re-suspended in $100 \mu \mathrm{L}$ PBS. The samples were heated at $95^{\circ} \mathrm{C}$ for 10 minutes before being stored at $-20^{\circ} \mathrm{C}$ until they could be analysed on a $12.5 \%$ SDS-PAGE gel.

\subsubsection{Full Scale Induction}

For protein purification, BL21 expressing the correct plasmid were cultured overnight. The resulting culture was used to inoculate $250 \mathrm{~mL}$ of kanamycin supplemented LB at a starting $\mathrm{OD}_{600}$ of 0.1 . Once an $\mathrm{OD}_{600}$ of $0.3-0.6$ was reached, a $1 \mathrm{~mL}$ un-induced sample was removed and centrifuged at $16,100 \times \mathrm{g}$ for 1 minute to pellet, before setting aside. Protein expression was induced with IPTG for 4 hours at $37^{\circ} \mathrm{C}$. After induction the $\mathrm{OD}_{600}$ was re-measured, and 1 $\mathrm{mL}$ induced sample was collected for the induced sample, before the remaining culture was centrifuged at 5,000 $\times g$ for 10 minutes. The resulting cell pellets from the induced samples were lysed with $4 \mathrm{~mL}$ B-Per (Thermo Scientific) per gram of pellet, according to the manufacturer's instructions. Both un-induced and induced pellets were re-suspended in PBS as previously described. 


\subsubsection{SDS-PAGE}

\subsubsection{Recipes}

\section{X SDS Loading Dye}

- 250 mM Tris- $\mathrm{HCl}$ pH 6.8

- $100 \mathrm{~g} / \mathrm{L}$ SDS $(10 \% \mathrm{w} / \mathrm{v})$

- Glycerol (30\% v/v)

- Bromophenol blue $(0.02 \% \mathrm{v} / \mathrm{v})$

\section{$\underline{\text { SDS 10X Running Buffer }}$}

- $30 \mathrm{~g} / \mathrm{L}$ Tris base

- $144 \mathrm{~g} / \mathrm{L}$ glycine

- $10 \mathrm{~g} / \mathrm{LSDS}$

\subsubsection{Protocol}

SDS-PAGE gels (12.5\%) were made using the TGX FastCast ${ }^{\mathrm{TM}}$ Acrylamide Kit (Bio-Rad) as per the manufacturer's instructions summarised here.

Resolving solutions were made up by mixing $8 \mathrm{~mL}$ of Resolver $A$ and Resolver B solutions together, followed by the addition of $10 \%$ APS ( $80 \mu \mathrm{L})$ and TEMED. $(8 \mu \mathrm{L})$ Stacking solutions were made up by mixing $2 \mathrm{~mL}$ of Stacker A and Stacker B solutions together, followed by $10 \%$ APS $(20 \mu \mathrm{L})$ and TEMED $(4 \mu \mathrm{L})$.

Alternatively, samples were run on pre-cast $8-16 \%$ Novex Tris-Glycine SDS-PAGE gels (Life Technologies). SDS-PAGE samples were made up by adding $\beta$-mercaptoethanol (0.7 M final concentration) and 5X SDS loading dye to protein samples at a 1:4:15 ratio. Samples were heated at $95^{\circ} \mathrm{C}$ for 10 minutes before loading onto the gel. Gels were run at $125 \mathrm{~V}$ for $45-90$ minutes. Following the run, gels were washed with $\mathrm{dd}_{2} \mathrm{O} 3$ times before staining with BioSafe pre-mixed Coomassie stain (Bio-Rad) for an hour. This was followed by de-staining in $\mathrm{ddH}_{2} \mathrm{O}$ for 30 minutes. 


\subsubsection{Protein Extraction and Purification}

The bacterial lysates containing His-tagged Bep proteins were applied to equilibrated Pierce HisPur-Ni-NTA $1 \mathrm{~mL}$ spin columns (Thermo Scientific), as per the manufacturer's instructions with some modifications, summarised below.

All steps were kept at $4^{\circ} \mathrm{C}$ where possible. Protein extracts were equilibrated by adding an equal volume of equilibration buffer (PBS with $10 \mathrm{mM}$ imidazole). Equilibration buffer $(2 \mathrm{~mL})$ was used to wash the $1 \mathrm{~mL}$ resin columns before adding the prepared protein extracts. The columns were mixed on an orbital shaker for 30 minutes at $4^{\circ} \mathrm{C}$, before centrifuging to collect the flow through. The resin was washed three times with $2 \mathrm{~mL}$ wash buffer (PBS and $20 \mathrm{mM}$ imidazole). The purified proteins were then eluted three times with $1 \mathrm{~mL}$ elution buffer (PBS and $200 \mathrm{mM}$ imidazole). All fractions, including the purified lysate and wash buffer flowthrough, as well as both un-induced and induced samples, were run on $12.5 \%$ SDS-PAGE gels to check for correct protein isolation, as well as successful purification. To regenerate the resin, the manufacturer's instructions were followed whereby the resin was washed with MES buffer $(10 \mathrm{~mL})$, followed by ultrapure $\mathrm{H}_{2} \mathrm{O}(10 \mathrm{~mL})$. The resin was stored as a $50 \%$ slurry in $20 \%$ ethanol.

Protein eluates were desalted using Microcon-10kDa centrifugal filter columns (Millipore). Each elution sample $(500 \mu \mathrm{L})$ was run on an individual column and spun at $14,000 \times g$ for 10 minutes. This was repeated until all elution samples had been loaded onto the column. To each column, $500 \mu \mathrm{L}$ of HEPES buffer ( $20 \mathrm{mM}, \mathrm{pH} 7.5$ ) was added and the column re-spun at $14,000 \times g$ for 10 minutes to dialyse the buffer. This was repeated to ensure effective buffer transfer. The protein was eluted by inverting the column into a new collection tube and spinning at $1,000 \times g$ for 2 minutes. Resulting protein solutions were stored at $4^{\circ} \mathrm{C}$ for up to a week before use. 


\subsubsection{Protein Quantification}

Protein quantification was performed using the Pierce BCA Protein Assay kit (Thermo Scientific) as per the manufacturer's instructions for microplate samples.

Unknown samples were diluted 1:10 and 1:100 in $\mathrm{ddH}_{2} \mathrm{O}$ or HEPES buffer and compared with diluted HEPES for background. Each standard or unknown sample replicate $(25 \mu \mathrm{L})$ was pipetted into a microplate well and $200 \mu \mathrm{L}$ of the BCA working reagent (WR) was added (giving a 1:8 sample to WR ratio). The solutions were mixed by shaking for 30 seconds and the plate was incubated for 30 minutes at $37^{\circ} \mathrm{C}$ before being cooled to ambient temperature. The absorbance was measured at $562 \mathrm{~nm}$ on a plate reader (EnSpire 2300 Multilabel Reader [Perkin Elmer]). The average value for the blank was subtracted from the standards and unknowns and a standard curve was prepared to determine the protein concentration of the unknown samples. All standards and unknowns were analysed in triplicate.

\subsection{Cell Culturing}

\subsubsection{Initiation and Maintenance of HUVEC Cell Culture}

Human Umbilical Vein Endothelial Cells (HUVEC; Life Technologies) were stored in liquid nitrogen until use. Cells were thawed by dipping the lower half of the vial into a $37^{\circ} \mathrm{C}$ water bath. From the vial $1 / 3^{\text {rd }}$ of the contents was pipetted into $25 \mathrm{~mL}$ of Medium 200 (Life Technologies) supplemented with Low Serum Growth Supplement (Life Technologies) in a T75 $(250 \mathrm{~mL})$ culture flask. This step was repeated two more times for the remaining HUVEC solution. Cultures were incubated in a $37^{\circ} \mathrm{C}, 5 \% \mathrm{CO}_{2}$ humidified cell culture incubator. Culture media was changed every $2-3$ days by removing the media, and pipetting fresh supplemented Medium $200(25 \mathrm{~mL})$ into the culture flask.

\subsubsection{Sub-culturing HUVECs}

Once HUVEC growth had reached $80-90 \%$ confluence, cells were sub-cultured by first removing the culture media from the flask. The cells were treated with trypsin by adding 4 $\mathrm{mL}$ trypsin-EDTA, spreading it over all cells, and then removing $3 \mathrm{~mL}$ of trypsin. After $1-2$ minutes of incubation, flasks were rapped to dislodge the HUVECs from the flask. Once the cells were dislodged, supplemented Medium $200(10 \mathrm{~mL})$ was added to the solution. From this a $1 \mathrm{~mL}$ sample was removed and added to supplemented Medium 200 (24 mL) in a new T75 flask resulting in a 1:10 dilution of cells. 


\subsubsection{Initiation and Maintenance of HeLa Culture}

Liquid nitrogen stocks of HeLa cells were thawed and cultured in T75 flasks in RMPI 1640 media (Life Technologies) supplemented with $10 \%$ foetal calf serum and $1 \%$ penicillinstreptomycin solution $\left(10,000 \mathrm{U} / \mathrm{mL}\right.$, Life Technologies). Cultures were incubated in a $37^{\circ} \mathrm{C}$, $5 \% \mathrm{CO}_{2}$ humidified cell culture incubator. Culture medium was changed every $2-3$ days by removing the media and pipetting fresh supplemented RPMI $(25 \mathrm{~mL})$ into the culture flask.

\subsubsection{Sub-culturing HeLas}

Once HeLa growth had reached $90-100 \%$ confluence, cells were sub-cultured by first removing the culture media from the flask. Cells were rinsed with EDTA/Trypsin solution, before treating with $2-3 \mathrm{~mL}$ EDTA/Trypsin solution. Once the cells had rounded (after roughly 5 minutes), flasks were rapped to dislodge attached cells from the flask. Supplemented RPMI 1640 medium ( $8 \mathrm{~mL}$ ) was added to resuspend the cells; $1 \mathrm{~mL}$ of this suspension was transferred to $24 \mathrm{~mL}$ supplemented RPMI 1640 in a new T75 flask.

\subsubsection{Whole Cell Extract Preparation}

Whole cell extracts were prepared for both HUVEC and HeLa cells using a method adapted from Lewallen et al. (2012). Cells were grown in $75 \mathrm{~mL}$ flasks to $90 \%$ confluence, with roughly $1 \times 10^{7}$ cells per flask.

Adherent cells were removed from the flask with a cell scraper in $10 \mathrm{~mL}$ sterile PBS. Suspended cells were transferred to two $50 \mathrm{~mL}$ centrifuge tubes (three flasks per tube). The cells were centrifuged at 2500 rpm for 3 minutes to pellet, before being washed with $10 \mathrm{~mL}$ of PBS. This step was repeated once more. The cells were then re-suspended gently in $500 \mu \mathrm{L}$ RIPA buffer (50 mM Tris-HCl pH 8.0 (HUVEC) or 20 mM HEPES pH 7.4 (HeLa), 150 mM NaCl, 1\% Triton X-100, 0.05\% deoxycholate, $10 \mu \mathrm{L} / \mathrm{mL}$ Halt Protease Inhibitor Cocktail [Thermo Scientific]) and incubated on ice for 30 minutes at $4^{\circ} \mathrm{C}$ on a orbital shaker. The resulting solution was then transferred to a $1.5 \mathrm{~mL}$ centrifuge tube and centrifuged at $13,400 \times \mathrm{g}$ for 20 minutes. The supernatant, which contained the whole cell extract, was transferred to a separate tube. HUVEC whole cell extracts $(500 \mu \mathrm{L})$ were concentrated using Microcon-10kDa centrifugal filter columns (Millipore), by spinning for 10 minutes at 14,000 x g. Extracts were eluted by spinning the column upside down in a fresh tube for 2 minutes at 1,000 $\mathrm{g}$. This concentration step was omitted for HeLa whole cell extracts. Protein concentration of all cell extracts was quantified as previously described. 


\subsection{Labelling Reactions}

\subsubsection{F1-ATP Methodology}

Whole cell extracts were immediately combined with purified recombinant Bep proteins, as well as a labelled ATP analogue, following their purification, to identify AMPylated host cell proteins. Purified recombinant Bartonella Bep proteins (100 $\mu \mathrm{g} / \mathrm{mL}$ final concentration) were combined with F1-ATP (N6-(6-amino)-hexyl-ATP-5-FAM; Jena Bioscience), a commercially available fluorescently labelled ATP derivative, and added to roughly $400 \mu \mathrm{g}$ of HUVEC cell extracts in Reaction buffer (20 mM HEPES pH 7.5, $100 \mathrm{mM} \mathrm{NaCl}, 1 \mathrm{mM} \mathrm{DTT}, 5 \mathrm{mM} \mathrm{MgCl}$ ) in a total volume of $100 \mu \mathrm{L}$ to give a final concentration of $60 \mu \mathrm{M}$ F1-ATP. A negative control containing no recombinant protein was also included. The reactions were incubated at $37^{\circ} \mathrm{C}$ for 90 minutes. Samples were run on pre-cast $8-16 \%$ Novex Tris-Glycine SDS-PAGE gels (Invitrogen) alongside a BenchMark Fluorescent Protein Standard (Life Technologies), a fluorescent protein marker. Gels were visualised using a Fuji fluorescent gel scanner with a FITC setting, followed by Coomassie staining.

\subsubsection{Click Chemistry Methodology}

The purified bacterial protein of interest $(100 \mu \mathrm{g} / \mathrm{mL}$ final concentration) was combined with $N^{6}$ pATP ( $\mathrm{N}^{6}$-propargyl-adenosine-5'-triphosphate; Jena Bioscience; $100 \mu \mathrm{M}$ final concentration), a commercially available ATP derivative, and incubated with $200-500 \mu \mathrm{g}$ of HeLa cell lysates for 1 hour at $30^{\circ} \mathrm{C}$, in a total volume of $100 \mu \mathrm{L}$ of $20 \mathrm{mM} \mathrm{HEPES}, \mathrm{pH} 7.4,100$ $\mathrm{mM} \mathrm{NaCl}, 5 \mathrm{mM} \mathrm{MgCl} 2$ and $1 \mathrm{mM}$ DTT. Purified Fic domain proteins, $V$. parahaemolyticus VopS and B. rochalimae Bep2, were included as positive controls. A mutant of $B$. henselae BepA, with the critical Fic domain histidine mutated (BhBepA_H159A-Fic) was included as a negative control. A sample with no added recombinant bacterial protein was also included. Reactions were stopped by addition of methanol $(400 \mu \mathrm{L})$ and chloroform (100 $\mu \mathrm{L})$, and the samples vortexed. Water $(300 \mu \mathrm{L})$ was added, and the samples vortexed again. Samples were centrifuged at $16,100 \times g$ for 5 minutes at ambient temperature. Following centrifugation, the upper phase was removed, $-20^{\circ} \mathrm{C}$ acetone $(1 \mathrm{~mL})$ was added, and the samples were vortexed. Samples were centrifuged at $23,000 \times g$ for 10 minutes at $4^{\circ} \mathrm{C}$ to pellet protein. The supernatant was removed, and pellets allowed to air dry for $5-10$ minutes. Dried pellets were re-suspended in $100 \mu \mathrm{L}$ 4\% SDS in 20 mM HEPES. 
A Click Chemistry mastermix was made up as follows: $22.3 \mu \mathrm{L}$ of 100 mM CuSO (Jena $^{2}$ Bioscience) was added to $877.35 \mu \mathrm{L}$ of 4\% SDS in 20 mM HEPES, followed by $55.75 \mu \mathrm{L} 200 \mathrm{mM}$ THPTA (Tris(3-hydroxypropyltriazolylmethyl)amine; Jena Bioscience). To the resulting solution, 44.6 $\mu \mathrm{L} \quad 10 \mathrm{mM}$ 6-FAM-azide (N-(3-azidopropyl)-3',6'-dihydroxy-3-oxo-3Hspiro[isobenzofuran-1,9'-xanthene]-6-carboxamide; Jena Bioscience) in DMSO was added, resulting in a final volume of $1 \mathrm{~mL}$. The resulting click chemistry Mastermix (90 $\mu \mathrm{L})$ was added to the samples. Click chemistry was initiated with the addition of $10 \mu \mathrm{L}$ of $100 \mathrm{mM}$ sodium ascorbate (Jena Bioscience). This resulted in a final reaction concentration of $1 \mathrm{mM} \mathrm{CuSO}_{4}, 5$ $\mathrm{mM}$ THPTA, $200 \mu \mathrm{M}$ 6-FAM azide and $5 \mathrm{mM}$ ascorbate. The reaction mixtures were incubated at $37^{\circ} \mathrm{C}$ for 1 hour in the dark. Reactions were stopped by addition of SDS sample buffer, with remainder being stored at $4^{\circ} \mathrm{C}$. SDS-PAGE samples were loaded onto pre-cast $8-16 \%$ Novex Tris-Glycine SDS-PAGE gels (Life Technologies) and run for 90 minutes at $125 \mathrm{~V}$ alongside a BenchMark Fluorescent Protein Standard (Life Technologies). Gels were visualised on a Fuji fluorescent gel scanner with a FITC setting, before Coomassie staining.

\subsection{Vimentin Polymerisation Assay}

Vimentin polymerisation was performed using a method adapted from Icenogle et al. (2012). Purified His-Bep protein $(100 \mu \mathrm{g} / \mathrm{mL}$ final concentration) was combined with ATP (1 mM final concentration; ThermoFisher Scientific), and added to $0.05 \mu \mathrm{g} / \mu \mathrm{L}$ of recombinant Syrian hamster vimentin (Cytoskeleton, Inc.) in $20 \mathrm{mM} \mathrm{HEPES}, 100 \mathrm{mM} \mathrm{NaCl}, 1 \mathrm{mM} \mathrm{DTT}, 5 \mathrm{mM} \mathrm{MgCl} 2$ $(500 \mu \mathrm{L})$. The reaction was incubated at $37^{\circ} \mathrm{C}$ for 3 hours. Negative controls were run with BhBepA_H159A-Fic mutant and no Bep protein respectively. Following incubation, samples were reserved for liquid chromatography-mass spectrometry/mass spectrometry (LCMS/MS) and pyrophosphate assay analysis.

The reactions were split into two equal aliquots. Vimentin polymerisation was induced in one sample by adding $\mathrm{NaCl}$ to a final concentration of $150 \mathrm{mM}$, while $\mathrm{ddH}_{2} \mathrm{O}$ was added to the other. Samples were incubated at ambient temperature for 60 minutes. Polymerised filaments were separated from soluble monomer subunits by ultracentrifugation (Airfuge; Beckman Coulter) at $80,000 \times g$, at $20^{\circ} \mathrm{C}$ for 90 minutes. The supernatant (100 $\left.\mu \mathrm{L}\right)$ was collected and each sample was run on pre-cast $8-16 \%$ Novex Tris-Glycine SDS-PAGE gels (Life Technologies) for 90 minutes at $125 \mathrm{~V}$, before being subjected to western blotting. 


\subsubsection{Western Blotting (WB)}

\subsubsection{Recipes}

\section{Transfer Buffer}

- $25 \mathrm{mM}$ Tris Base

- 192 mM Glycine

- $20 \%(\mathrm{v} / \mathrm{v})$ Methanol

- $0.01 \%(w / v)$ SDS

\section{TBS (Tris-Buffered Saline) 10X}

- $200 \mathrm{mM}$ Tris Base

- $1.5 \mathrm{M} \mathrm{NaCl}$

- Adjust pH to 7.6

\section{$\underline{\text { TBS-T 1X }}$}

- $100 \mathrm{~mL}$ TBS $10 \mathrm{X}$

- $900 \mathrm{~mL} \mathrm{ddH_{2 } \mathrm { O }}$

- $0.1 \%(\mathrm{v} / \mathrm{v})$ Tween 20

\section{Blocking Buffer}

- $8 \%(\mathrm{w} / \mathrm{v})$ milk powder in TBS-T

\subsubsection{Protocol}

Protein gels were removed from electrophoresis equipment and soaked in cold transfer buffer for $10-30$ minutes. Nitrocellulose membranes (pore size $0.2 \mu \mathrm{m}$; Thermo Scientific) were cut to match gel size, then soaked in cold transfer buffer until use. Filter paper was cut to size, then briefly soaked in cold transfer buffer before assembling western blot sandwich.

Protein samples were transferred to a nitrocellulose membrane at $30 \mathrm{~V}$ for 90 minutes on ice. Membranes were washed with blocking buffer for $2-3$ hours at ambient temperature on a shaker. Following blocking, membranes were transferred to a $5 \mathrm{~mL}$ solution of diluted primary antibody in TBS-T (1:500; mouse monoclonal [RV202] anti-vimentin antibody [Abcam]) and allowed to shake overnight at $4^{\circ} \mathrm{C}$. Membranes were washed four times for 7 minutes each with TBS-T buffer. TBS-T was discarded and $5 \mathrm{~mL}$ secondary antibody solution in TBS-T was added (1:2000; Goat anti-mouse IgG H\&L [Alexa Fluor ${ }^{\circledR} 647$; Abcam]) and allowed to shake in the dark for 1 hour at ambient temperature. 
Membranes were washed again as above and visualised on a Typhoon FLA 9500 Scanner (GE Healthcare Life Sciences) using an Alexa Fluor ${ }^{\circledR} 647$ setting.

\subsection{Pyrophosphate Detection/Bep activity assay}

To assess the recombinant Bep proteins for activity, sub-samples of the vimentin polymerisation assay were taken and analysed for pyrophosphate levels. Similarly, repeat experiments based on the F1-ATP labelling experiments were performed, with regular ATP (1 $\mathrm{mM}$; Thermo Scientific) substituting for F1-ATP, and with the reaction length being extended to 3 hours. To detect pyrophosphate production, the ab112155 Fluorometric Pyrophosphate Assay Kit (Abcam) was utilised as per manufacturer's instructions, summarised below:

PPi sensor stock (200X) solution was prepared by adding $50 \mu \mathrm{L}$ DMSO to lyophilised sensor. Assay sensor (1X) was prepared by diluting $10 \mu \mathrm{L}$ PPi sensor in $2 \mathrm{~mL}$ Assay Buffer. Pyrophosphate assays were performed by adding Assay Sensor (50 $\mu \mathrm{L})$ to $50 \mu \mathrm{L}$ of pyrophosphate standards or AMPylation samples diluted (1:10) in Assay buffer in a fluorescence 96 well plate and mixed thoroughly. The plate was then incubated at ambient temperature for 10-30 minutes. Fluorescence was measured on a plate reader (EnSpire 2300 Multilabel Reader [Perkin Elmer]) using excitation/emission wavelengths of 316/456 nm respectively. The resulting data was imported into and analysed by Microsoft Excel.

\subsection{LC-MS/MS Preparation}

Protein samples were taken from previous experiments and were precipitated using the ProteoExtract $^{\circledR}$ Protein Precipitation kit (Merck Millipore) as per the manufacturer's instructions for samples less than $200 \mu \mathrm{L}$. Resulting protein pellets were dissolved in $50 \mu \mathrm{L} 8$ M Urea/0.1 M Tris, pH 8.5, before being reduced by $0.2 \mathrm{M} \mathrm{DTT}(1.25 \mu \mathrm{L})$ and incubated at $56^{\circ} \mathrm{C}$ for 35 minutes. Samples were cooled to ambient temperature and subsequently alkylated with $500 \mathrm{mM}$ iodoacetamide $(1 \mu \mathrm{L})$ in the dark for 30 minutes. Proteins were diluted 3-fold with $0.1 \mathrm{M}$ Tris, $\mathrm{pH} 8.5$ to bring total urea concentration down to $2 \mathrm{M}$. $\mathrm{CaCl}_{2}$ solution was added ( $1 \mathrm{mM}$ final concentration), and resulting solutions were added to $1 \mu \mathrm{g}$ of trypsin each. Samples were digested overnight at $37^{\circ} \mathrm{C}$ and $200 \mathrm{rpm}$. The reactions were quenched with $90 \%$ formic acid and stored at $-20^{\circ} \mathrm{C}$ until ready for further purification with Bond Elut OMIX tips. 


\subsubsection{Sample Preparation with Bond Elut OMIX Tips}

Bond Elut OMIX micro extraction tips (Agilent) were pre-wet with a $1: 1$ solution of $0.4 \%$ trifluoroacetic acid (TFA)/acetonitrile ( $A C N$ ) by aspirating three times, one tip per protein sample. OMIX tips were then equilibrated with a $1: 1$ solution of $0.4 \% \mathrm{v} / \mathrm{v} T F A / \mathrm{H}_{2} \mathrm{O}$. To bind protein to OMIX tips, protein solutions were aspirated 20 times, before rinsing the OMIX tip with $0.4 \% \mathrm{TFA} / \mathrm{H}_{2} \mathrm{O}$. Protein was eluted into $20 \mu \mathrm{L} 0.1 \%$ formic acid in $70 \% \mathrm{ACN} / \mathrm{H}_{2} \mathrm{O}$. Resulting elutions were concentrated to $10 \mu \mathrm{L}$ volume in a SpeedVac vacuum centrifuge (Labconco), before storing at $4^{\circ} \mathrm{C}$ until use.

To prepare samples for loading onto the LC-MS, samples were mixed with $70 \mu \mathrm{L}$ of Buffer $\mathrm{A}$ ( $0.1 \%$ formic acid in $\mathrm{H}_{2} \mathrm{O}$ ), vortexed, then centrifuged for 2 minutes. Most of the supernatant was pipetted into a GC vial with an insert.

\subsection{Liquid Chromatography and Mass Spectrometry}

LC-MS/MS experiments were performed by Dr Lifeng Peng using a Dionex UltiMate ${ }^{\circledR} 3000$ RSLCnano System, coupled to a LTQ Orbitrap XL mass spectrometer, via a nanospray ion source (Thermo Fisher Scientific, USA). Peptides were separated on a $75 \mu \mathrm{m} \times 15 \mathrm{~cm}$ PepMap C18 column ( $3 \mu \mathrm{m}, 300 \AA$ Dionex) at a flow rate of $300 \mathrm{~nL} / \mathrm{min}$. The LC gradient was constructed from Buffer A (0.1\% formic acid) and Buffer B (0.1\% formic acid in $80 \%$ ACN): $2 \%$ Buffer B to start, followed by a linear gradient from 2-22\% B for over 65 minutes, followed by a linear gradient from $22-45 \%$ B for over 35 minutes, followed by a linear gradient from $45-95 \%$ B for 20 minutes, before being held at $95 \%$ for 5 minutes.

The spray voltage was set at $2.4 \mathrm{kV}$, and the temperature of the heated capillary was set at $200^{\circ} \mathrm{C}$. Full MS scan (m/z $\left.200-1850\right)$ in profile mode was acquired in the Orbitrap with 30,000 resolution. The six most intense peptide ions from the full scan were selected and fragmented using CID (normalised collision energy, 35\%; activation Q, 0.250; and activation time, 30 milliseconds). Dynamic exclusion was enabled with the following settings: repeat count, 2; repeat duration, 30 seconds; exclusion list size, 500; exclusion duration, 90 seconds. The spectra were acquired using Xcalibur (version 2.1.0 SP1, Thermo Fisher Scientific). 


\subsubsection{Data Analysis}

Protein identification was conducted using the search algorithm SEQUEST under Proteome Discoverer (version 1.4.1.14, Thermo Scientific). All searches were performed with two databases; one containing only vimentin, another containing the UniProt all human database as of $12 / 02 / 16$. The database was indexed with trypsin as the proteolytic enzyme, 'fully enzymatic' as the enzyme limit, with a molecular weight range of $300-5000 \mathrm{kDa}$, and 2 missed cleavage sites. SEQUEST searches were conducted for $B$ and $Y$ ions only, with dynamic protein modifications of 329.052 and 330.060 monoisotopic masses for AMP de-protonated and protonated forms respectively. AMPylation was analysed for serine, threonine, tyrosine, and histidine residues. Oxidation, carbamyl, deamidated, and carbamidomethyl dynamic modifications were also included in the analysis. 


\section{Labelling Whole Cell Extract with F1-ATP}

\subsection{Introduction}

\subsubsection{Bacterial Effectors}

Pathogenic bacteria have evolved a wide range of toxins and effector proteins that are able to subvert host cell processes and cause disease. By studying these toxins and effector proteins, a greater understanding of disease progression may be obtained. This can lead to a wide range of treatments and interventions including potential vaccine antigens and novel antibiotic targets. Similarly, bacterial toxins and effectors can serve as tools for studying normal cellular processes including signalling, cytoskeleton rearrangements, and plasma membrane permeability ${ }^{90,91}$. Many bacterial effector proteins mimic natural host cell proteins such as the GEF (guanine nucleotide exchange factor), and GAP (GTPase activating protein) motif effectors, which regulate host GTPase protein activity ${ }^{91,92}$.

Increasingly, AMPylation has been shown to be a post-translational modification utilised by bacterial effectors to manipulate host cell processes ${ }^{66,71,75,84}$. In particular, several Fic domaincontaining bacterial effectors have been shown to AMPylate host cell proteins, including the Rho GTPases and vimentin. AMPylation often leads to interference with the normal function of the target protein. Many Bartonella species contain several Fic domain-containing proteins, although their precise roles in disease or transmission remain unknown.

\subsubsection{Previous AMPylation Studies}

Several groups working on Fic domain effector proteins from other bacterial pathogens have successfully identified their host AMPylation targets ${ }^{66,71,75,84}$. Published work identifying targets of Fic domain effectors have used a number of approaches, the most common being the use of radiolabelled $\left[\alpha^{32} \mathrm{P}\right] \mathrm{ATP}^{71,75,80,84}$. While this method is very effective it has drawbacks, requiring special training and disposal of the radioactive material, and presenting difficulties in identification of the AMPylated (i.e. radiolabelled) protein ${ }^{88}$. Another published method makes use of antibodies against AMPylated threonine or AMPylated tyrosine ${ }^{93}$. While this approach does not require radioactivity and allows for immunoprecipitation of labelled protein to enable easier identification, the downside is that there are currently no commercially available antibodies to AMPylated serine. This approach could potentially miss novel targets, such as the BiP chaperone, which is AMPylated on a serine residue by the endogenous Fic domain protein, $\mathrm{HYPE}^{78}$. Problems can also arise with non-specific binding ${ }^{94}$. 
An alternative method proposed by Lewallen et al. (2012) uses an ATP derivative with a covalently linked fluorophore. One such molecule is F1-ATP (Figure 3.1). Experiments involving the Vibrio parahaemolyticus VopS Type III effector and whole cell extracts demonstrated that VopS could label known substrates with this ATP analogue, and that target proteins could be easily identified and excised from a SDS-PAGE when viewed under a FITC setting. The benefit of this type of probe is that it is less toxic than radiolabelling and enables immunoprecipitation using anti-FITC antibodies. A potential drawback to this method is that the incorporation of a bulky fluorophore on the ATP substrate may potentially interfere with the Fic proteins ability to catalyse the AMPylation reaction. Despite the potential drawbacks, this method was selected to identify the host cell target proteins of the Bartonella Beps studied here.

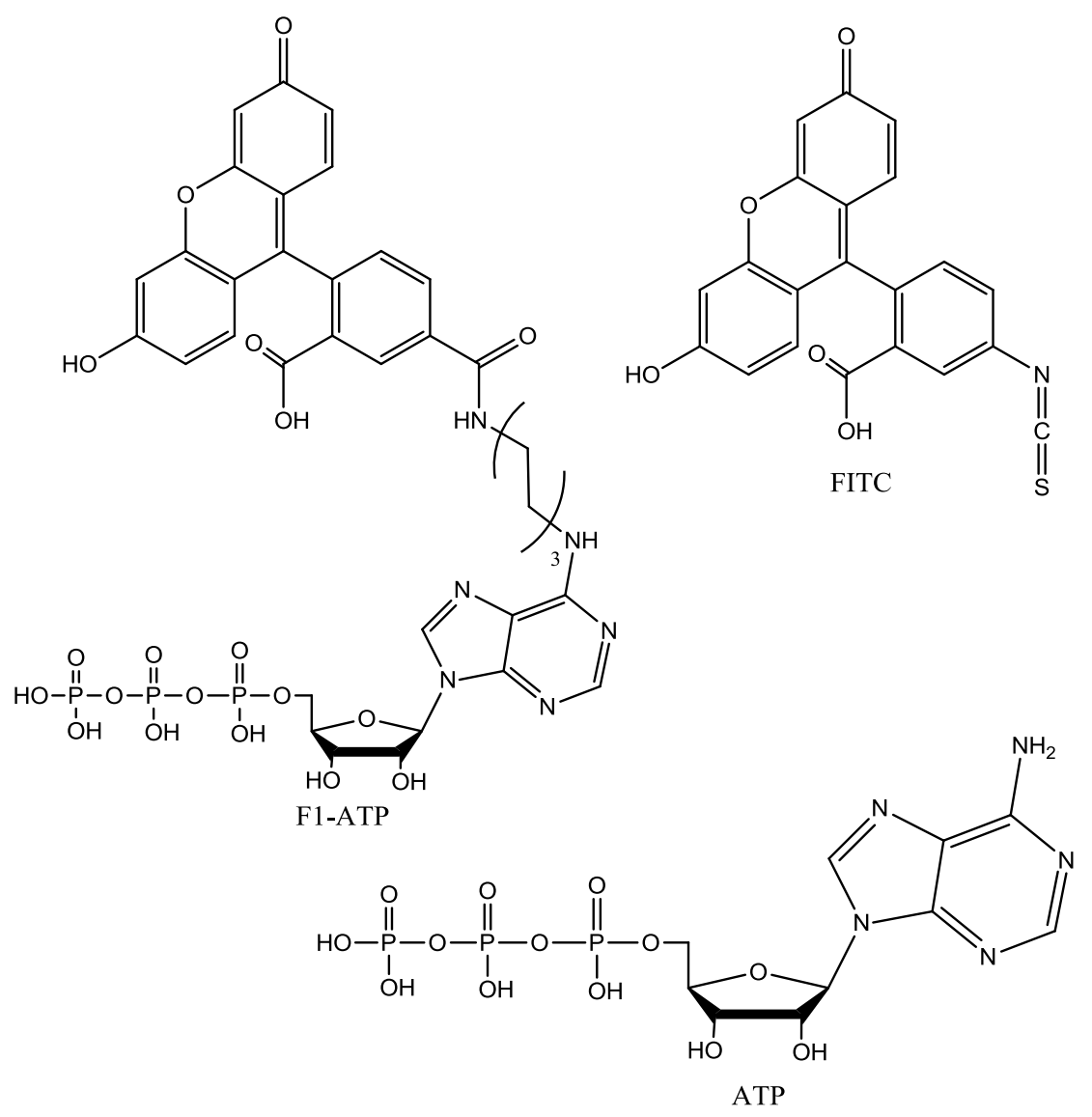

Figure 3.1: Structural comparison of F1-ATP, FITC, and ATP. 


\subsubsection{Construct Design}

To identify the host target proteins, fragments of the Bartonella Fic domain proteins were expressed and purified in an exogenous host (E. coli). Recombinant proteins were truncated to omit the BID domain, and contained an $\mathrm{N}$-terminal $\mathrm{His}_{6}$ tag to enable purification by nickelaffinity chromatography. An $\mathrm{N}$-terminal, over a C-terminal His ${ }_{6}$-tag, was chosen to retain the option of removing the $\mathrm{His}_{6}$-tag by thrombin cleavage.

Plasmids were constructed to enable expression and purification of the Fic domains of $B$. henselae proteins BepA (NCBI - BH13370), BepB (NCBI - BH13390), and BepC (NCBI BH13400), originally described by Schulein et al. (2005), as well as the B. quintana proteins BepA1 (NCBI - BQ10630) and BepC (NCBI - BQ10650), originally described by Alsmark et al. (2004). BepA primers were designed to amplify the first 909 base pairs of the bepA gene, as this corresponds with the first 303 amino acids of the protein. Palanivelu et al. (2011) have shown that a similarly truncated BepA protein (10-303) had functional Fic protein activity, and was capable of auto-AMPylation, as well as AMPylation of two unidentified HeLa cell proteins. The BepA fragment length was designed to exclude expression of the Bartonella Intracellular Delivery (BID) domain of the C-terminal of the protein. BID domains are highly conserved in many Bartonella species Bep proteins and have several of their own functions. For example, the BepA BID domain enables T4SS translocation of BepA into the host cell, where it inhibits host cell apoptosis ${ }^{60}$. By omitting the BID domain, any enzymatic activity detected is likely attributable to the Fic domain of the protein.

A similar methodology was applied for the bepB and bepC genes. Pulliainen et al. (2012) described the starting point of the BID domain in B. henselae Beps $A, B$, and $C$ and this was used as a reference when constructing the primers. Primers were designed to amplify only the first 894 base pairs of bepB, and the first 855 base pairs of bepC. Both BepB and BepC have BID domains that occur earlier in the protein than BepA, with BepC being the shortest (Figure 3.2). 


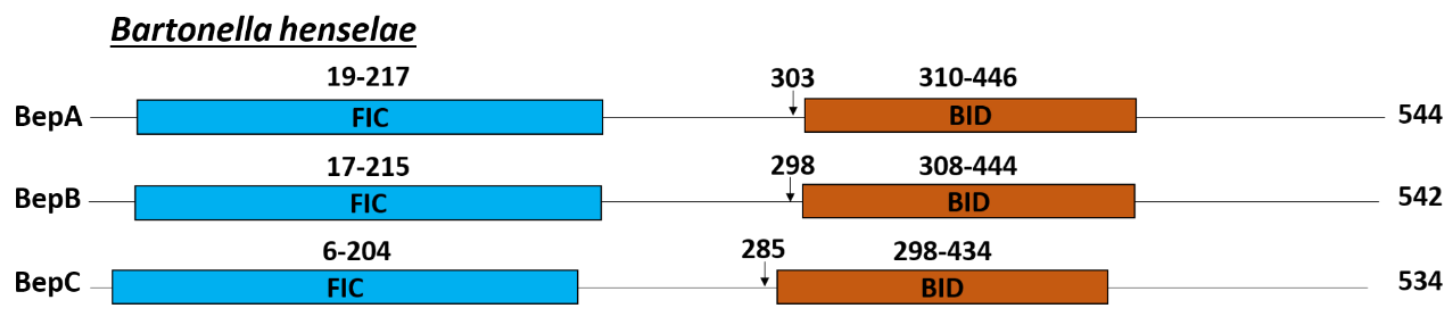

\section{Bartonella quintana}

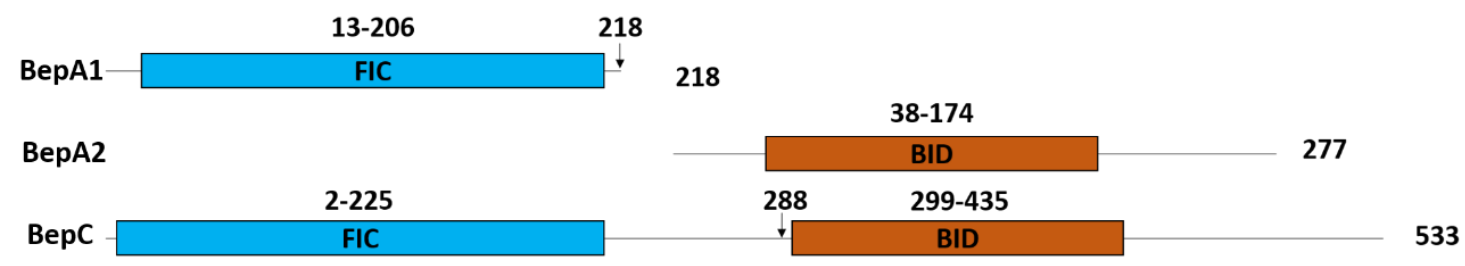

Figure 3.2: Bartonella effector proteins studied. The arrows show the ends of the fragments expressed in this study. In the case of B. quintana BepA1, the whole gene was cloned (B. quintana BepA2 is included for completeness).

In the case of the Bartonella quintana genes, the primers for bepA1 were designed to amplify the whole gene as the BID domain is expressed as a separate ORF (bepA2) ${ }^{60}$. For B. quintana bepC, the primers were designed to give as much coverage of the gene as possible while still excluding the BID domain. To do this, similarities to the $B$. henselae bepC were used as a reference, with the first 864 base pairs being cloned.

All of the plasmid sequences were confirmed to be correct by aligning with NCBI sequences, using the Geneious 8.1.7 Multiple Alignment function.

\subsubsection{Protein Expression}

The plasmid constructs were transformed into the BL21 E. coli host strain. Following induction of protein expression by IPTG, cells were lysed and recombinant proteins were purified by nickel affinity chromatography. Of the 5 protein constructs described, all were shown to be induced in BL21 cells, but only BhBepA-Fic, and BhBepB-Fic proteins could be successfully isolated (Appendix Section 9.2). Comparison of the soluble and insoluble fractions from cell lysis revealed that the $\mathrm{BhBepC}$-Fic, BqBepA1, and $\mathrm{BqBepC}$-Fic protein fragments were largely insoluble. Unfortunately, time constraints precluded resolution of these insolubility issues. 


\subsection{Results}

\subsubsection{Optimisation of Protein Recovery from Whole Cell Extract}

Before F1-ATP labelling experiments could be undertaken, optimisation of whole cell extract recovery was required as initial attempts resulted in very low levels of protein $(150 \mu \mathrm{g} / \mathrm{mL})$. In order to increase protein concentration of whole cell extract, three flasks of HUVEC cultures were scraped and combined into a single Falcon tube before lysing with RIPA buffer. The resulting lysate was further concentrated using a $10 \mathrm{kDa}$ Microcon column (Millipore). To further improve protein extraction, protease inhibitor was added to RIPA buffer. The results are shown in Figure 3.3 below. These results clearly indicate that both techniques were able to significantly increase protein concentration obtained in the whole cell extract.

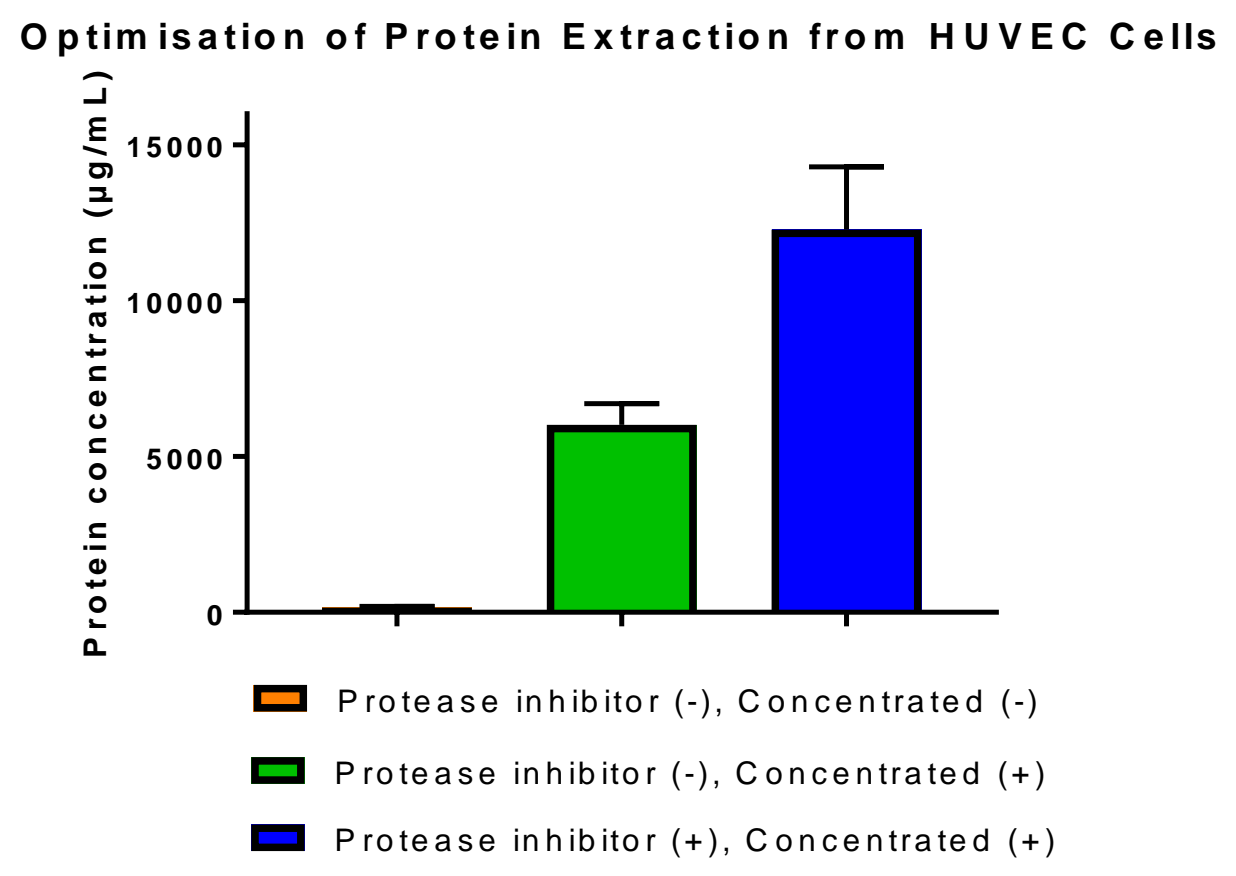

Figure 3.3: Optimisation of protein extraction from HUVEC cells. 


\subsubsection{F1-ATP Labelling}

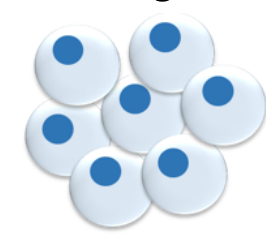

HUVEC cells

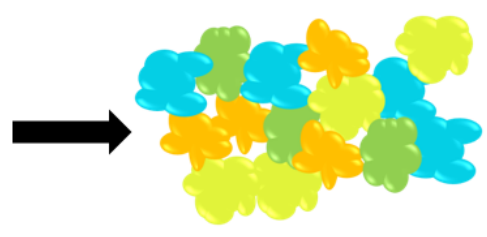

Whole Cell Extract
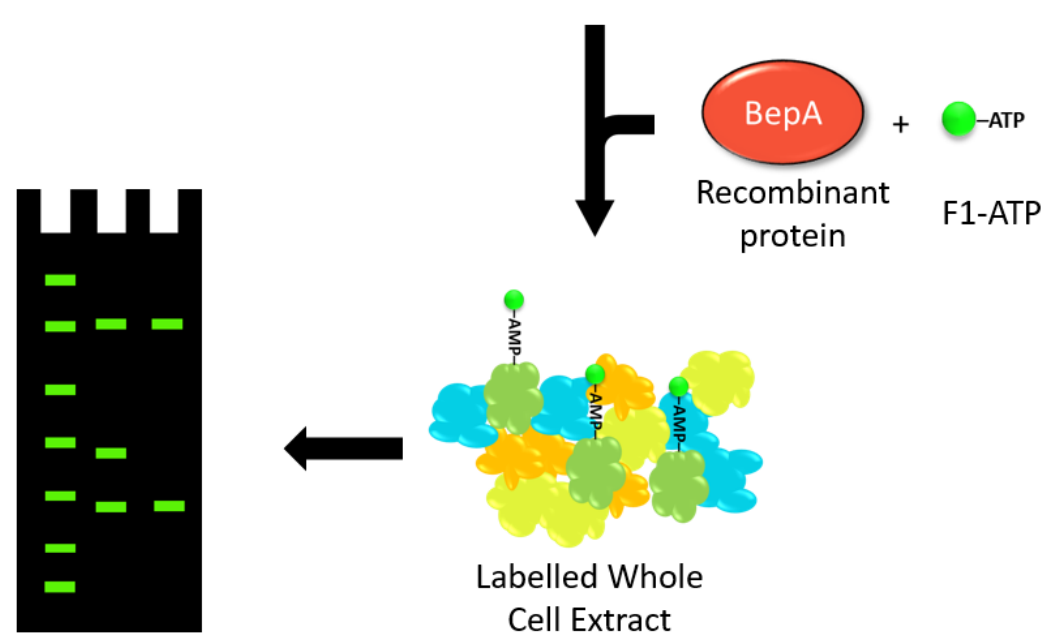

Fluorescent SDSPAGE gel

Figure 3.4: Simplified methodology of the F1-ATP labelling experiments. First, protein is extracted from the HUVEC cells (whole cell extract). This whole extract is treated with recombinant Fic-containing protein (BepA) and F1-ATP resulting in fluorescently labelled protein in the whole cell extract. The reaction sample is then run on a SDS-PAGE gel and analysed under a FITC setting. Novel bands are detected by comparison of sample (middle lane) with negative samples (right lane). Fluorescent marker is also included (left lane).

Whole cell extracts obtained from HUVECs were incubated at $37^{\circ} \mathrm{C}$ with purified recombinant Bep proteins, with F1-ATP added as a substrate, according to a method adapted from Lewallen et al. (2012). A negative control lacking recombinant proteins was included. Following incubation, proteins in the reaction mixture were run on an SDS-PAGE gel and visualised with a fluorescence gel scanner (Figure 3.4). The results are shown in Figure 3.5 below. Each reaction lane contains 3 bands, but no difference was observed between the BhBepA-Fic and BhBepB-Fic reactions and the negative control. This result indicates whole cell extract was not labelled by the recombinant Bep proteins. 


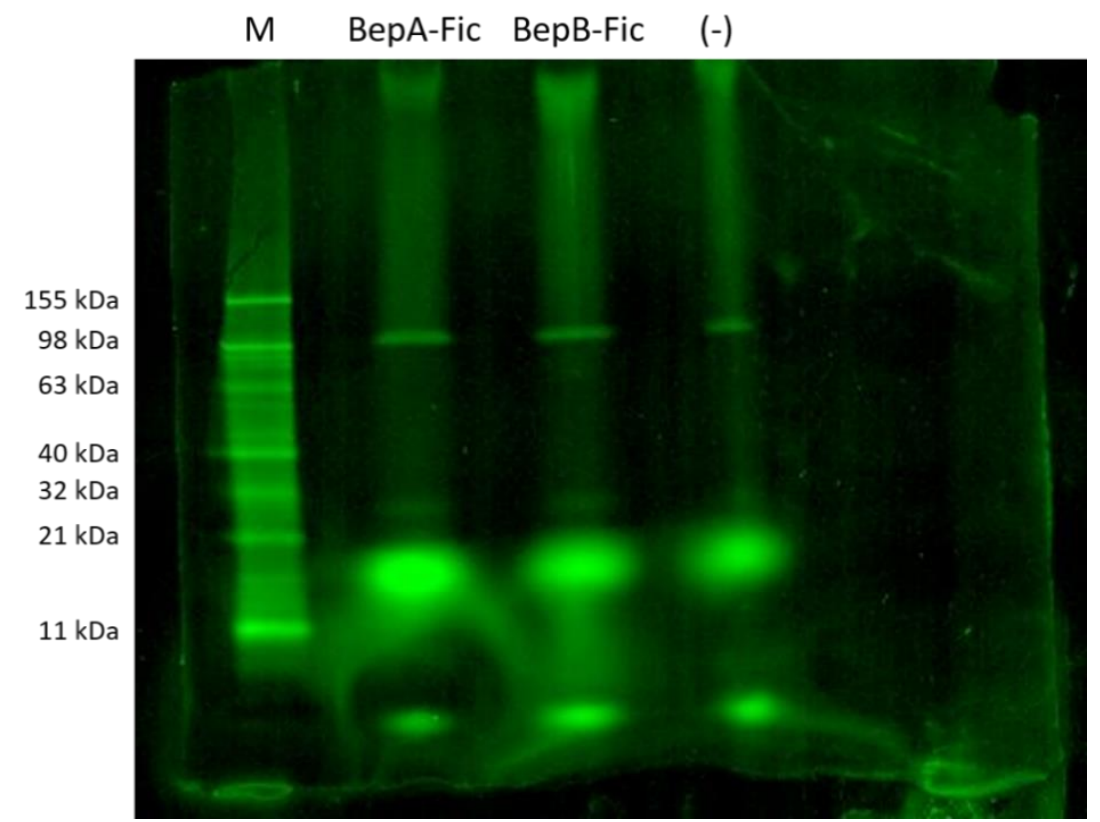

Figure 3.5: F1-ATP labelling results. No protein control indicated with a (-). Results show no difference between samples, indicating no AMPylation by recombinant Bep proteins. $M=$ Fluorescent marker, gel used was Novex 8-16\% (Life Technologies).

\subsection{Discussion}

The goal of this part of the project was to label whole cell extract from HUVECs and identify potential AMPylation target proteins of recombinant BepA and BepB from B. henselae. Unfortunately, the results from the F1-ATP labelling experiment were inconclusive, as there was no difference between the reactions with the recombinant Bep proteins and the negative control. The bands observed are likely the result of endogenous AMPylation protein activity in the whole cell extract.

There could be a number of factors to explain why no additional bands were observed in the reactions containing recombinant Bep proteins including steric hindrance from the large fluorophore attached to the ATP analogue. As stated by Lewallen et al. (2012), this ATP analogue decreased the rate of AMP transfer by a factor of 10 for VopS. It may also be the case that this substrate results in an even greater decrease in the rate of AMP transfer for BepA and BepB Fic domains. Another factor may be that the target proteins of these Beps are expressed at low levels in the whole cell extracts and was below the level of detection. Finally, the possibility that the recombinant protein fragments were catalytically inactive cannot be excluded. 
In order to optimise the experiment, an alternative labelling experiment was employed using a less sterically bulky ATP derivative, as well as positive control recombinant proteins.

\subsubsection{Protein Solubility}

Three of the proteins expressed in this study, B. henselae BhBepC-Fic, B. quintana BqBepA1, and $B$. quintana BqBepC-Fic, were unable to be purified successfully due to solubility issues. Despite a large amount of protein being expressed during the preliminary induction, the induced protein was only present in the insoluble fraction during full-scale induction. This indicates that the protein was likely present in inclusion bodies, large spherical particles made up of insoluble protein aggregates ${ }^{95}$. Inclusion body formation is usually the result of the cell's quality control system being unable to repair or remove misfolded or unfolded protein that rapidly accumulations during protein induction ${ }^{95}$. There are several potential factors for why these proteins were insoluble, some of which are described below.

One possible factor is the difference in codon usage between Bartonella species and E. coli. These two species vary significantly in their relative synonymous codon usage (RSCU) ${ }^{96,97}$. In particular, the only amino acids with similar RSCU between species are alanine, valine, lysine, glutamine and arginine, along with methionine and tryptophan, which have only a single codon. Target genes containing significant numbers of rare codons particularly in the initiator sequence recognised by the ribosome, are more likely to experience translational stalling in E. coli, which could result in low level expression or truncated protein ${ }^{95,98}$. However, preliminary analysis suggests that all proteins expressed had similar levels of rare codons (around $15-21 \%$ of codons). The protein constructs also contained an $\mathrm{N}$-terminal His 6 tag, resulting in an initiator sequence that is designed to suit E. coli. For these reasons it seems unlikely this is the cause of the solubility issues.

Another factor that could contribute to protein solubility is the presence of large regions of contiguous hydrophobic residues, particularly at the ends of proteins ${ }^{98}$. Although all of the expressed proteins lacked contiguous hydrophobic regions at the $\mathrm{N}$ and $\mathrm{C}$ termini, previous studies have found that shifting either the $\mathrm{N}$ or $\mathrm{C}$ terminus of the recombinant protein fragment by as little as 4 amino acids, can have a large effect on protein solubility ${ }^{98,99}$. As our experiments used recombinant protein fragments, shifting the C-terminus may have resulted in increased solubility. 
For future experiments, it may be beneficial to test recombinant protein fragments of several lengths for solubility. There is also the possibility that omission of the BID domain in both BepC-Fic proteins, and the removal of the oligonucleotide/oligosaccharide binding fold in BqBepA1, contributed to loss of solubility. In particular, the full $B$. henselae BepC protein has previously been expressed, and shown to induce invasome formation along with $\mathrm{BepF}^{51}$, indicating that the protein is soluble, and active, when expressed at full length. Future experiments could look at several methods for increasing solubility of the recombinant proteins before trialling new constructs with the C-terminus shifted, such as those discussed in Chapter 6. 


\section{Click Chemistry}

\subsection{Introduction}

\subsubsection{Copper-catalysed azide-alkyne cycloaddition (CuAAC)}

As the F1-ATP labelling experiment did not result in identification of host cell target proteins, an alternative methodology was required. The copper-catalysed azide-alkyne cycloaddition (CUAAC) is the most widely recognised example of click chemistry ${ }^{100}$, and provides an alternative to F1-ATP labelling. First described in 2002, it has been used in an expanding range of applications across several different fields. The selective reactivity of azides and alkynes with each other allows CUAAC to be used for the manipulation and labelling of biomolecules of interest, including proteins ${ }^{100}$. This high selectivity of CUAAC avoids side reactions, and has the potential to aid in detection of AMPylated substrates.

Grammel et al. (2011) utilised CuAAC to develop a novel alkyne substrate for identifying AMPylated protein targets. N6 pATP is a derivative of ATP, coupling the $\mathrm{NH}_{2}$ at the 6 position of adenosine with a propargyl group (Figure 4.1). This $\mathrm{N}^{6}$ position was selected based on structural data available for a number of Fido domain-containing proteins, including VopS, IbpA, and $\mathrm{BepA}^{102}$, thus making it an appropriate ATP analogue for this experiment. The propargyl group can be coupled to various azide molecules, including fluorescent azides and streptavidin-coupled azides, allowing for labelling proteins post AMPylation reaction (Figure 4.2). This has the benefit of omitting large, bulky fluorophores from the AMPylation reaction, as these may have inhibited the F1-ATP labelling reactions. Click chemistry has been used to demonstrate that both VopS and IbpA label distinct proteins in HeLa whole cell extracts, which are consistent with the molecular weight of small GTPases (roughly $21 \mathrm{kDa})^{94,101}$.

While Grammel et al. (2011) describe a click chemistry methodology, other groups have published improvements to the basic copper-catalysed azide alkyne cycloaddition, including optimised reagents ${ }^{100,103}$. Originally TCEP (tris(2-carboxyethyl)phosphine) was described as the reducing agent of choice; however, recent evidence has found that Cu-binding and azidereducing properties of phosphines can inhibit the CUAAC reaction ${ }^{103}$. For this reason, ascorbate has been proposed as an alternative reducing agent. Another advance was the incorporation of the accelerating ligand THPTA. Previously, the non-water soluble ligand TBTA (Tris[(1-benzyl-1H-,1,2,3-triazol-4-yl)methyl]amine) had been used, requiring co-solvents such as DMSO and n-butanol ${ }^{100,101,103}$. 
The amount of THPTA required for a successful CUAAC reaction must be 5 equivalents relative to copper ${ }^{100}$. The reason is to intercept and quickly reduce reactive oxygen species that may be generated by ascorbate driven reduction of oxygen present in the reaction mixture. As ascorbate can undergo side reactions with dissolved oxygen, it is important an excess is used relative to copper. Presolski et al. (2011) describe the use of $5 \mathrm{mM}$ ascorbate, which was also used here

Another important factor to consider when performing the CuAAC reaction is the presence of interfering compounds in the reaction buffer. Tris buffer must be avoided during the click chemistry reaction, as Tris will compete with THPTA for copper binding ${ }^{100,103}$. Similarly, chloride ions above $0.2 \mathrm{M}$ also compete for copper binding ${ }^{103}$. For these reasons Tris- $\mathrm{HCl}$ was replaced with HEPES in the RIPA buffer, and salt concentration during the reactions was strictly controlled.
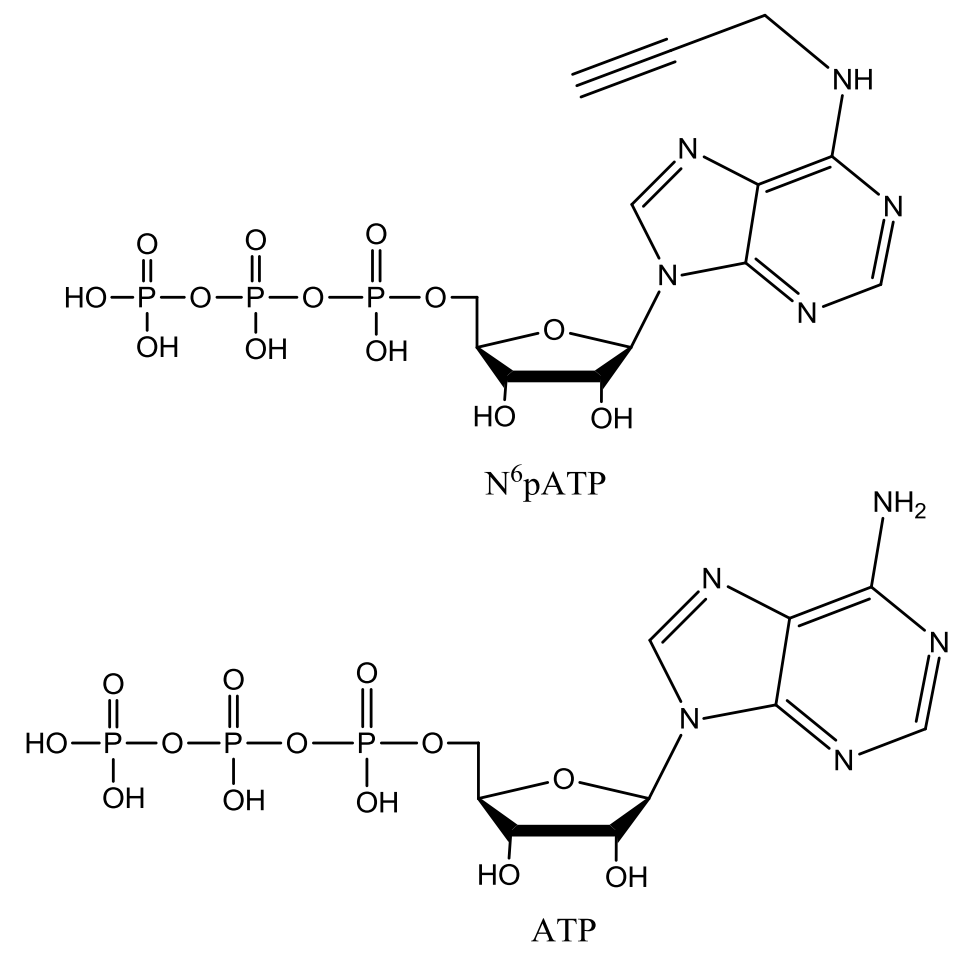

Figure 4.1: Structural comparison of $\mathbf{N}^{6} \mathrm{pATP}$ and ATP. A propargyl group has been added to the $\mathrm{N}^{6}$ position of the regular ATP molecule. 

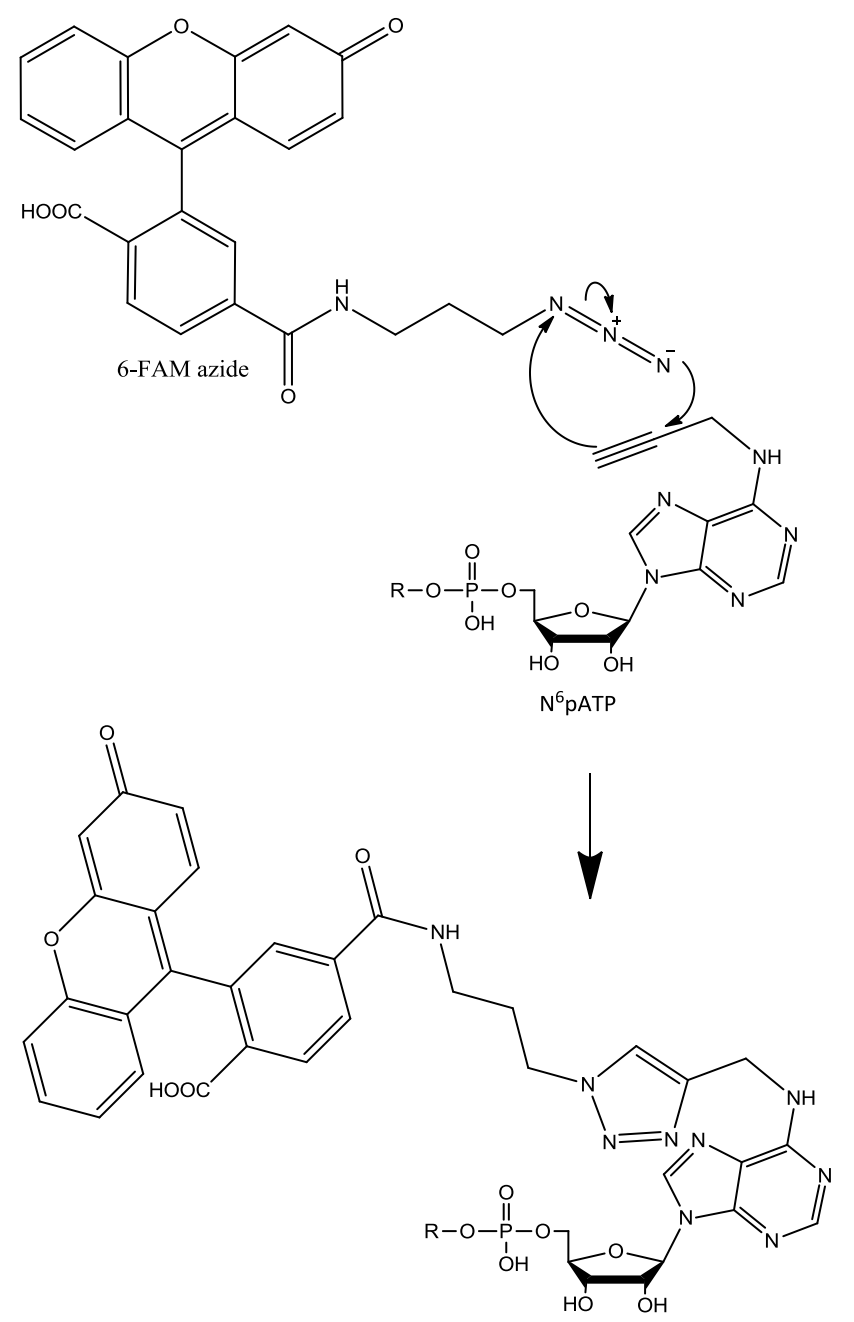

Figure 4.2: Basic reaction scheme for the CuAAC click chemistry reaction. The reaction involves a 1,3-dipolar cycloaddition forming a 1,2,3 triazole structure. In this example, the reagents used in the experiments are pictured with 6-FAM azide representing the azide, and $\mathrm{N}^{6} \mathrm{pATP}$ representing the alkyne. The $\mathrm{R}$ group represents the labelled protein.

\subsubsection{VopS and Bep2-Fic Control Constructs}

In order to ensure the labelling reaction was working correctly, positive control proteins were purified. VopS from Vibrio parahaemolyticus is the best studied Fic-containing protein and has previously been shown to react with $\mathrm{N}^{6} \mathrm{p}$ ATP and label a target protein in whole cell extracts $^{101}$. Similarly, Bep2 from B. rochalimae has also demonstrated AMPylation activity ${ }^{84}$. In particular, Bep2 is related to the Beps studied here, making it an ideal control protein. To produce recombinant proteins without needing to import and culture Vibrio parahaemolyticus or Bartonella rochalimae, gBlocks from IDT-DNA were designed and ordered, with the resulting DNA fragments cloned into the pET28a vector.

VopS and BrBep2-Fic sequences were confirmed to be correct by aligning with NCBI sequences, using the Geneious 8.1.7 Multiple Alignment function. 


\subsubsection{BepA H159A Mutant}

A catalytically inactive $B$. henselae BhBepA-Fic mutant protein was also constructed to include as a negative control for labelling assays. This mutant had the critical and invariant histidine residue of the Fic domain (His159) substituted by an alanine. The site-directed mutation was confirmed by sequencing (Appendix Figure 9.1).

\subsection{Results}

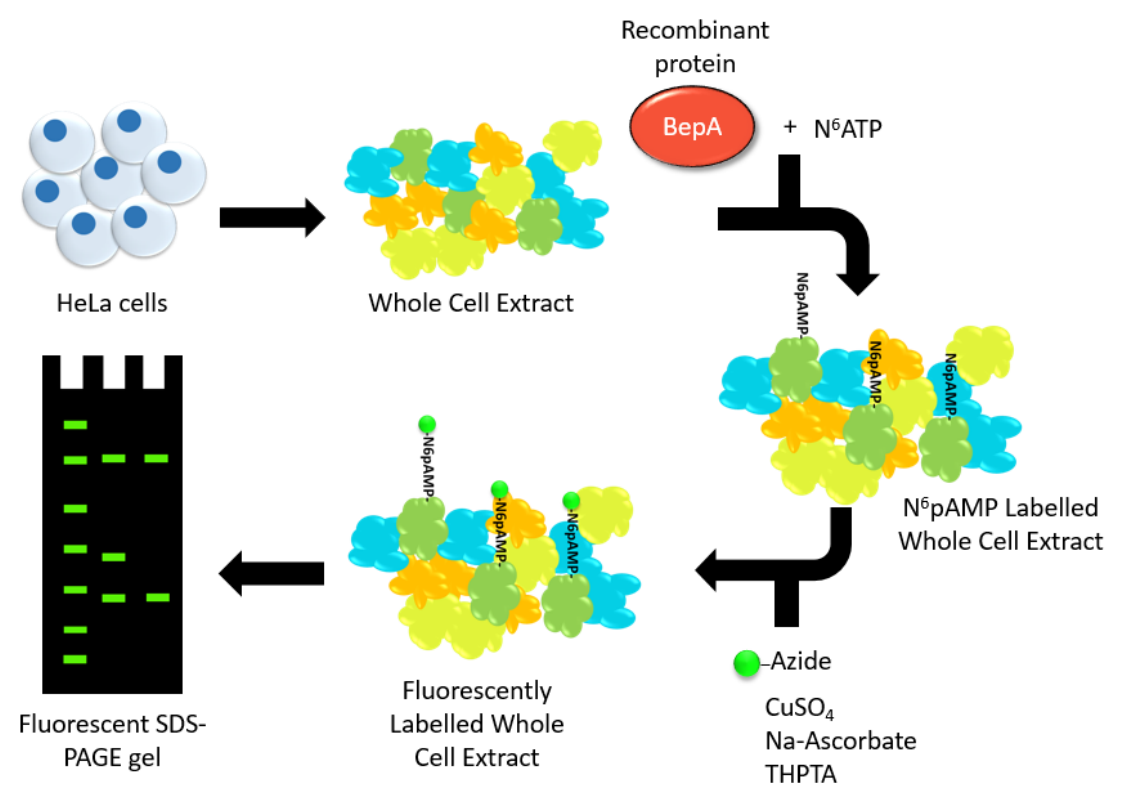

Figure 4.3: Simplified methodology of the click chemistry labelling experiments. First, protein is extracted from the HeLa cells (whole cell extract). This whole cell extract is treated with recombinant Fic-containing protein (BepA) and N6 $\mathrm{N}^{6} \mathrm{ATP}$, before subsequently coupling a fluorescent azide to the AMPylated proteins using click chemistry. This results in labelled protein in the whole cell extract. The reaction sample was then run on a SDS-PAGE gel and analysed under a FITC setting. Novel bands were detected by comparison of sample (middle lane) with negative samples (right lane). Fluorescent marker is also included (left lane).

Purified recombinant $B$. henselae Bep proteins, along with recombinant VopS and BrBep2-Fic positive controls, were added to a solution containing whole cell extracts and $\mathrm{N}^{6} \mathrm{pATP}$ and incubated at $30^{\circ} \mathrm{C}$. Following incubation, the total protein was precipitated with methanol/chloroform/water, and subjected to treatment with click chemistry reagents. Samples were run on an SDS-PAGE gel and visualised on a gel scanner.

A sample without any added purified protein was included as a negative control. To confirm the role of the Fic enzymatic domain, a Fic catalytic mutant, BhBepA_H159A-Fic, was also included as a control. 


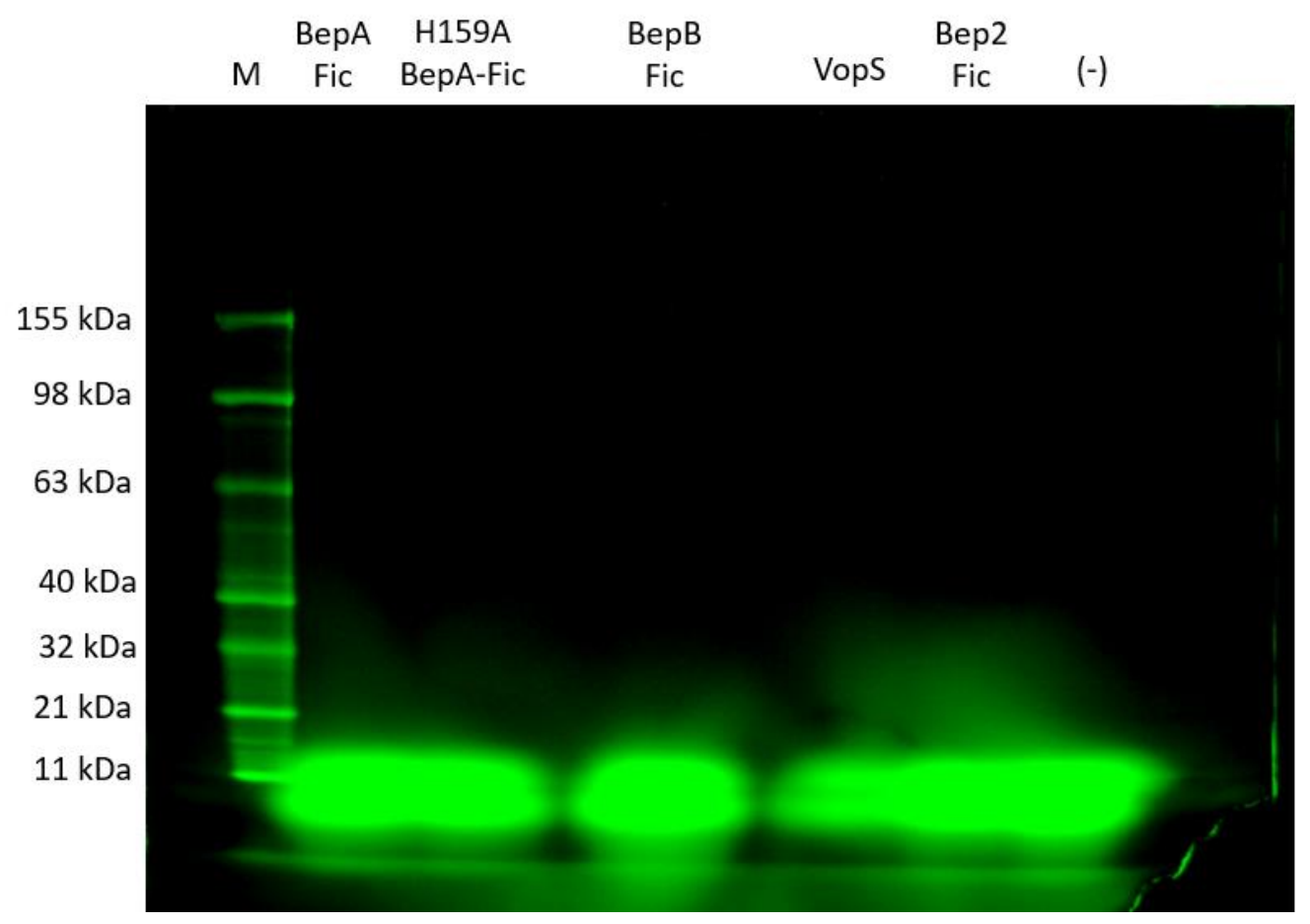

Figure 4.4: Results from the Click Chemistry Experiment. No protein control indicated with a (-). Results show no difference between samples, indicating no labelling by recombinant Fic-containing proteins. Two bands appear at the bottom of the gel, one just above the other. $M=$ Fluorescent marker, gel used was Novex 8-16\% (Life Technologies).

The results (Figure 4.4) show that similar sized bands were obtained for each of the samples, including both positive and negative controls. All of the lanes had two fluorescent bands of similar size at the bottom of the gel. The bands are likely the unreacted azide, as well as azide that has coupled to any $N^{6}$ pATP that remained during the protein purification step. This suggests that the click chemistry reaction did occur, although no labelled protein bands were observed, and would explain why the band occurs at a slightly higher molecular weight than unreacted azide. Unlike the results observed for the F1-ATP reactions, no background proteins were labelled by endogenous AMPylation enzymes present in the whole cell extracts. This could be due to differences between the $\mathrm{N}^{6} \mathrm{pATP}$ and F1-ATP substrates, although this seems unlikely as $N^{6}$ pATP is less sterically bulky than F1-ATP.

A more likely explanation is the difference in endogenous proteins expressed in HUVEC and HeLa cells, which represent endothelial and epithelioid cells, respectively. 


\subsection{Discussion}

The goal of this experiment was to develop an improved methodology over the experiments using F1-ATP as a substrate, to label HeLa whole cell extracts and identify protein targets of the Fic domain $B$. henselae Bep recombinant proteins. This click chemistry methodology was adapted from the work by Grammel et al. (2011), while incorporating the advances in click chemistry ${ }^{100,101,103}$. This methodology was developed to reduce the potential impact of a bulky fluorophore on the ATP analogue, and to determine whether the negative results were due to inactive recombinant proteins. To optimise the experiment, different concentrations of $\mathrm{CuSO}_{4}(0.25 \mathrm{mM}$ or $1 \mathrm{mM})$ and THPTA $(1.25 \mathrm{mM}$ or $5 \mathrm{mM})$ were trialled, along with differing incubation lengths for both the AMPylation and subsequent CuAAC experiment. Similarly, the AMPylation reaction was optimised by incubation $30^{\circ} \mathrm{C}$, rather than $37^{\circ} \mathrm{C}$, as used with the F1ATP experiment. Previous studies of Fic domain proteins VopS, Bep2, and BepA have described reaction incubations at $30^{\circ} \mathrm{C}^{66,84,101}$.

Unfortunately, no bands were identified in either the VopS or BrBep2-Fic controls, or the BhBepA-Fic and BhBepB-Fic samples, despite optimising reaction conditions. Click chemistry reactions were trialled for several incubation times, from 60 minutes up to overnight, while reactions were carried out at temperatures ranging from ambient to $37^{\circ} \mathrm{C}$. AMPylation reactions with $\mathrm{N}^{6} \mathrm{pATP}$ were also trialled for 60 and 90 minutes at $30^{\circ} \mathrm{C}$. No differences were observed for any of these reactions, suggesting additional time does not benefit either the click chemistry or AMPylation reactions. While the concentrations reported appear to give an effective click reaction, experiments utilising lower concentrations of $\mathrm{CuSO}_{4}(0.25 \mathrm{mM})$ and THPTA (1.25 mM) also gave similar results. Based on these results and those obtained from the F1-ATP labelling experiments, we concluded that the purified protein constructs were inactive. The limited time allowed for this research project precluded extensive optimisation of protein purification to retain activity. 


\section{Vimentin, Pyrophosphate, and MS Assays.}

\subsection{Introduction}

\subsubsection{B. rochalimae Bep2 and Vimentin}

While the functions of many of the Bartonella species effector proteins are unknown, the host cell target protein of Bep2, from the recently discovered human pathogen B. rochalimae ${ }^{83}$, has been identified by Pieles et al. (2014). Unlike B. henselae and B. quintana, B. rochalimae is a member of the Group B Bartonella species $^{37}$. While there are significant differences between Groups B and C, such as the Trw T4SS in Group C, and the flagella in Group B, there are also some similarities. Both Groups contain a least one VirB/D4 T4SS and associated Bep effector proteins. Furthermore, there is increasing evidence of gene transfers occurring between these two Bartonella Groups ${ }^{37}$. The phylogenetic relationship between $B$. rochalimae Bep2, the B. henselae Beps, and other Bartonella Fic-containing proteins, discussed in Chapter 1, is illustrated in Figure 5.1. From the phylogenetic tree it is clear that the $B$. henselae Beps separate into two distinct categories: those containing Fic domains, and those without. The tree also demonstrates that both BepC proteins from $B$. henselae and $B$. quintana are more distantly related to the other Fic-containing Beps, including Bep2 from $B$. rochalimae. Interestingly, B. rochalimae Bep2 clusters closely with BepA1 from B. quintana, which consists solely of the Fic domain, and lacks a BID domain. Pairwise alignment of BepA from $B$. henselae with Bep2 from B. rochalimae reveals that the largest difference between these two proteins is in the BID domains of the proteins. Similar results are observed between BepB from B. henselae and Bep2 from B. rochalimae.

Using a mass spectrometry based approach, with stable isotopically labelled ATP molecules, Bep2 was confirmed to target vimentin, a $50 \mathrm{kDa}$ type III intermediate filament protein of the cytoskeleton, in mouse macrophage cells ${ }^{84}$. Vimentin is also found in human mesenchymalderived cells and may also be a target protein of $\mathrm{BepA}^{85}$.

This hypothesis was supported by Palanivelu et al. (2011) who found that a BepA Fic fragment radioactively labelled an unknown $50 \mathrm{kDa}$ protein in S100 HeLa cell lysate. Vimentin shows a high degree of sequence morphology between species, and has a role in several cellular functions, including maintenance of the integrity of the vascular endothelium ${ }^{85}$. 
This suggests that vimentin may play an important role in the ability of Bartonella to colonise endothelial cells, and could be a potential target of B. henselae and B. quintana Fic-containing Beps.

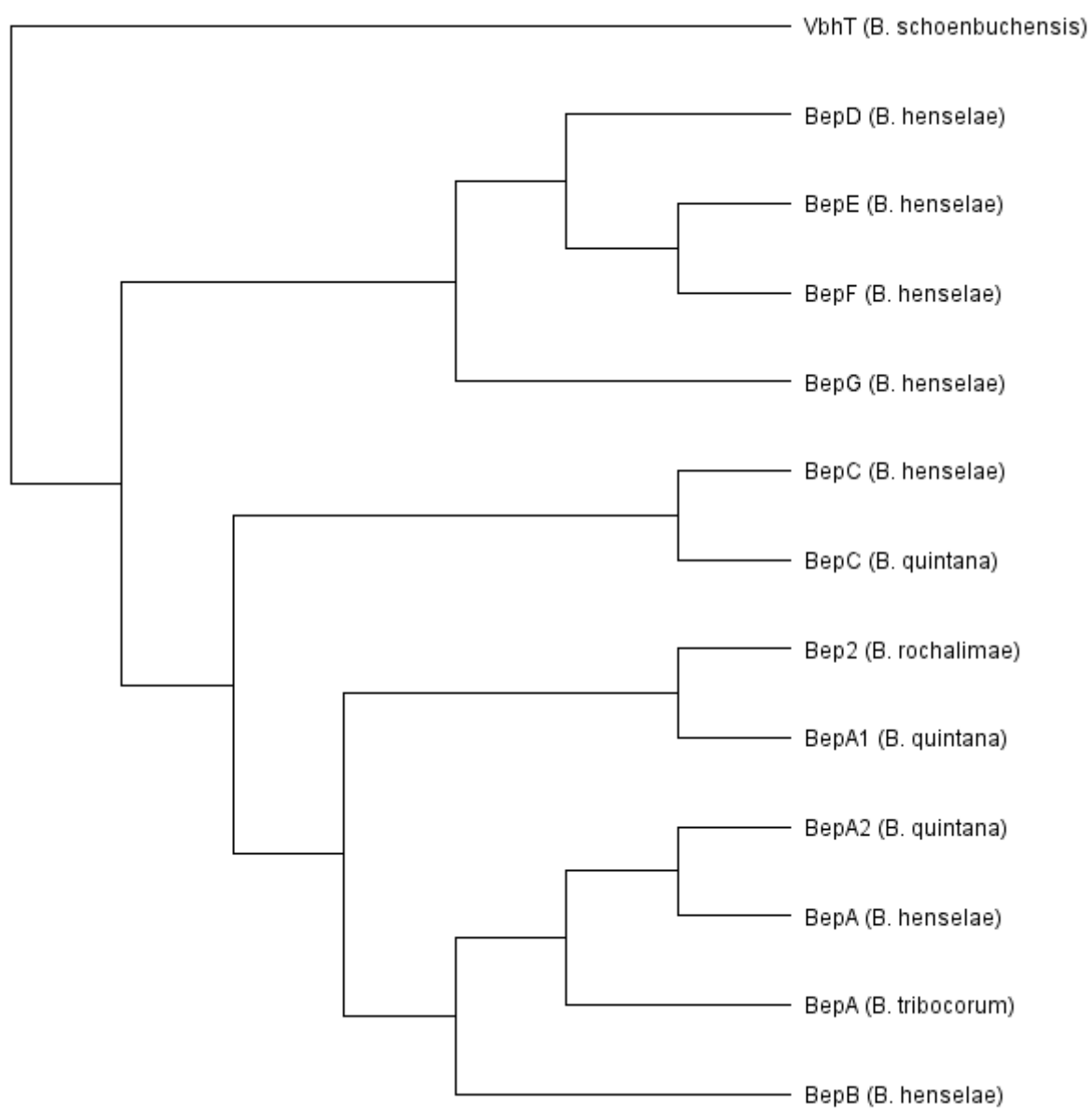

Figure 5.1: Cladogram of protein sequences for major Bartonella Bep proteins. The cladogram contains Beps used in this study and other previous studies. A clear distinction is observed between Beps that contain a Fic domain, and those that don't. VbhT from B. schoenbuchensis was used as the outgroup. Cladogram was created using Geneious 8.1.7 with JukesCantor Genetic Distance Method using a Global Alignment Setting.

\subsubsection{SpyA and Vimentin Polymerisation}

Interestingly, Bep2 is not the only bacterial toxin shown to modify host vimentin by transferring a chemical group. SpyA, a toxin produced by the gram-positive human pathogen, Streptococcus pyogenes, modifies and inactivates host vimentin by ADP-ribosylation at the head domain 89,104 . Actin is also modified by SpyA, though to a lesser extent. ADP-ribosylation of vimentin by SpyA interferes in the polymerisation of vimentin monomers, leading to collapse of the vimentin cytoskeleton and defects in wound repair 89,104 . 
Icenogle et al. (2012) describe an in vitro experiment to detect inhibition of vimentin polymerisation. This method involves incubating recombinant hamster vimentin with SpyA and the enzyme substrate, $\mathrm{NAD}^{+}$. This was followed by splitting the reaction in half and inducing polymerisation in one sample with $\mathrm{NaCl}$, and leaving the other sample unpolymerised by adding only water. Samples were subjected to ultracentrifugation to separate polymerised vimentin from soluble vimentin monomers. Supernatants from all samples were then run on an SDS-PAGE gel before being subjected to western blotting using anti-vimentin antibodies. Samples treated with water show a single band that acts as a positive control for antibody binding. The presence of a band in the $\mathrm{NaCl}$ treated samples indicates that vimentin polymerisation was inhibited by the ADP-ribosylation reaction from SpyA. This assay could be adapted for the $B$. henselae recombinant Bep proteins.

\subsubsection{Pyrophosphate Analysis and Mass Spectrometry.}

The vimentin polymerisation assay allowed for analysis of recombinant $B$. henselae proteins, without competing reactions for ATP or interfering compounds from whole cell extract, and utilised ATP, rather than an analogue. However, there were several reasons why the assay might not work. For example, vimentin may not be a target of AMPylation, or, if it is, the AMPylation may not occur on a part of the protein that would result in inhibition of vimentin polymerisation. For these reasons other experiments were also carried out.

An alternative approach used was the detection of the by-products from the AMPylation reaction carried out by recombinant Fic proteins. Based on catalytic mechanisms (Figure 1.3, Figure 1.4), the AMPylation reaction also produces pyrophosphate. Measuring pyrophosphate levels in the buffer, following the AMPylation reaction represents an indirect way to detect AMPylation activity against vimentin in the reaction mixture.

There is also the possibility that the recombinant proteins had AMPylation activity against host cell extract proteins, but that this wasn't observed during the labelling experiments due to problems with the substrates. In order to test this, pyrophosphate assays were also performed against whole cell extract obtained from HUVECs.

For these reactions, the same method was used as for the F1-ATP experiments, except that F1-ATP was substituted with ATP, and the reaction was run for 3 hours at $37^{\circ} \mathrm{C}$. 
In addition, a mass spectrometry analysis of vimentin, and whole cell extract samples was also performed on reactions for pyrophosphate analysis. Mass spectrometry analysis was previously used to identify AMPylation of Cdc42 by Vops ${ }^{71}$. A similar technique could be used for vimentin, or even possibly whole cell extract samples, by detecting an increase in the mass of peptide fragments that corresponds with the mass of AMP.

\subsection{Results}

\subsubsection{Vimentin Polymerisation}

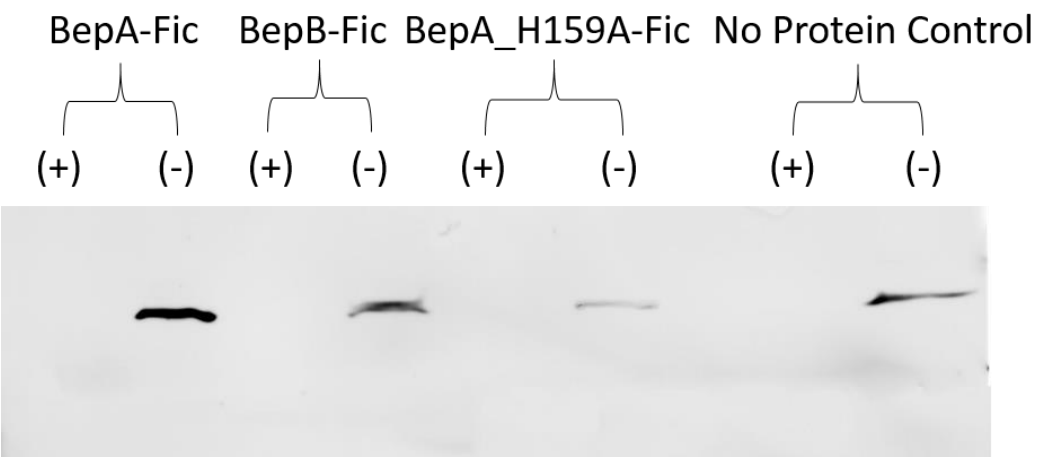

Figure 5.2: Vimentin Polymerisation Results. Polymerisation was induced by addition of $150 \mathrm{mM} \mathrm{NaCl}(+)$ or not induced by addition of water (-). No difference was observed for any of the four reaction samples (the fainter band for H159A was due to a dilution before running on the gel due to low solution volume remaining).

Recombinant Syrian hamster vimentin was combined in a reaction buffer with recombinant BhBepA-Fic and BhBepB-Fic proteins, along with ATP for 3 hours at $37^{\circ} \mathrm{C}$. Both the BhBepA_H159A-Fic catalytic mutant, and a sample without any added recombinant Fic protein, were included as negative controls. Following the reaction, half of each mixture was treated with $\mathrm{NaCl}$ to induce polymerisation. Polymerised vimentin was pelleted by ultracentrifugation. The supernatant was applied to an SDS-PAGE gel and subjected to western blot analysis. The presence of a band indicates that (non-polymerised) vimentin monomers were present; absence of a band indicates that the vimentin monomers were incorporated into polymerised vimentin, and removed by ultracentrifugation.

Figure 5.2 illustrates the results from western blot analysis. For all samples, polymerisation occurred when $\mathrm{NaCl}$ was added to the reactions, indicating that none of the recombinant Bep proteins were able to inhibit vimentin polymerisation. 
Although the BhBepA_H159A-Fic non-polymerisation sample contains a fainter band than the other samples, this was likely due to a dilution that occurred during the preparation, and was not considered significant. This result indicates neither the Fic domain of BepA or BepB inhibited vimentin polymerisation.

The click chemistry results indicated that no host cell proteins were labelled by BrBep2-Fic, suggesting that the purified recombinant protein may have been inactive. Therefore, the vimentin polymerisation assay was not carried out with this protein.

\subsubsection{Pyrophosphate Assay}

In order to test the recombinant B. henselae proteins BhBepA-Fic and BhBepB-Fic for activity, the amount of pyrophosphate present in these samples was quantified using the ab112155 fluorometric pyrophosphate kit (Abcam). This assay provides sensitive detection of pyrophosphate, detecting between 0.1-100 $\mu \mathrm{M}$ pyrophosphate. BepA_H159A-Fic, as well as a sample with no added recombinant Fic protein, were included as negative controls. AMPylation reactions were carried out against vimentin or whole cell extract using ATP (Thermo Scientific) for 3 hours at $37^{\circ} \mathrm{C}$. Pyrophosphate levels were measured post AMPylation reaction, and the difference in B. henselae BhBepA-Fic and BhBepB-Fic samples was compared against the negative controls to determine if these proteins displayed any AMPylation activity.

The assay results showed no significant change in pyrophosphate levels between BhBepA-Fic, BhBepB-Fic and negative controls for either the vimentin samples or the whole cell extract samples (Appendix Figure 9.16, Appendix Figure 9.17). This is consistent with the results from the polymerisation assay, and the previous labelling experiments, which did not detect AMPylation.

\subsubsection{LC-MS/MS}

There was a possibility that vimentin was AMPylated by one or both recombinant proteins, but that the AMPylation did not inhibit polymerisation. Similarly, it was possible that the pyrophosphate assay was not sensitive enough to detect minor differences in levels of pyrophosphate production. Both the vimentin and whole cell extract samples from the pyrophosphate analysis were, therefore, analysed by LC-MS/MS, and selected for peptides that had been covalently modified by an AMP moiety. 
Results from the LC-MS/MS analysis showed no evidence of AMPylation modification for any of the samples (results not shown). For the vimentin only samples, both databases did find top peptide matches to vimentin, confirming that MS analysis was correctly detecting and analysing peptide fragments.

The complexity of the whole cell extract samples made data analysis difficult to draw conclusions from, and suggests that the LC-MS/MS analysis is better suited for a smaller scale system, such as that from extracted bands in a gel, or a single protein assay, like that for vimentin, or $\mathrm{Cdc42}$, as described previously ${ }^{71}$. These results are consistent with those from previous experiments. In summary no evidence of vimentin modification, or AMPylation activity, by either BhBepA-Fic or BhBepB-Fic was detected.

\subsection{Discussion}

The goal of this part of the project was to determine if the B. henselae BhBepA-Fic and BhBepB-Fic recombinant proteins were able to AMPylate vimentin, and if this modification prevented polymerisation. The second goal was to determine if the recombinant Fic proteins displayed any AMPylation activity. The experimental results observed here all support those found by the F1-ATP and click chemistry labelling experiments, and support the hypothesis that the protein purification did not result in enzymatically active recombinant proteins.

\subsubsection{Vimentin Polymerisation}

The vimentin polymerisation reactions were initially performed without a positive control. BrBep2-Fic was selected as a positive control, however there were delays with purifying this protein. For this reason, a follow up experiment using BrBep2-Fic was planned. However, once the negative result from the click chemistry experiment was observed (section 4.2), the vimentin experiments were not repeated with BrBep2-Fic.

If solubility and purification of recombinant proteins can be improved to give active Fic domain proteins, including BrBep2-Fic, the experiment could be revisited. However, the effect of the AMPylation of vimentin by BrBep2-Fic on vimentin polymerisation is not known, so an alternative positive control may also be required. Previously SpyA has been shown to inhibit vimentin polymerisation by ADP-ribosylating the head region ${ }^{89}$. For this reason, a SpyA control reaction, with $\mathrm{NAD}^{+}$instead of ATP as the substrate, should also be included. 
In order to obtain recombinant SpyA, a gBlock for spyA could be constructed, as was done for vopS and bep2.

\subsubsection{Pyrophosphate assay}

The negative results from the pyrophosphate assay are consistent with the evidence suggesting the recombinant proteins are enzymatically inactive. There was however, a limitation with this assay. While the level of detection of pyrophosphate was sensitive to low levels of pyrophosphate, there was significant variation in fluorescence between replicates for both the standards and the samples. This variability made it difficult to draw conclusions from the results. Despite this limitation, detection of pyrophosphate production could be revisited if recombinant proteins can be purified in an active state. In particular, tests using recombinant vimentin and BrBep2-Fic or Cdc42 and VopS could be undertaken to test improved purification methods. Using these proteins with recombinant substrates would allow for testing in the absence of interfering factors that may be present in whole cell extract, and could give a good indication of activity for the other recombinant proteins studied here. Once protein purification is optimised, and target proteins identified by click chemistry, future experiments looking at kinetic studies of Fic-containing proteins using pyrophosphate assays could also be undertaken. This could potentially involve a similar set up to the vimentin and pyrophosphate experiments, with vimentin being substituted for the relevant target protein. This assay could then be performed over a 30 minute period, with the pyrophosphate levels being measured every five minutes in a 96 well plate reader. 


\section{Research Motivation, Key Findings, and Future Directions}

\subsection{Research Motivation}

With the recent discovery of the Fic domain function, there have been an increasing number of studies looking at the significance of this domain in bacterial proteins. In particular, Fic domains have been characterised in a number of secreted toxins from several bacterial pathogens including; Vibrio parahaemolyticus (VopS), Legionella pneumophilia (Ank), Histophilus somni (IbpA), and B. rochalimae (Bep2) ${ }^{71,74-76,84}$. For these proteins, both the covalent modification and the host interaction target have been described. These proteins all catalyse post-translational modifications, most commonly AMPylation of the Rho GTPase central signalling molecules, preventing them from binding downstream effectors and resulting in cytoskeletal collapse. In the case of VopS and IbpA, the catalytic mechanism has also been proposed ${ }^{79,80}$. Both $B$. henselae and $B$. quintana express Fic domain-containing proteins that are directly secreted into the host cell by a Type IV secretion system, although these haven't been as extensively studied. $B$. henselae expresses seven secreted effector proteins, three of which contain a Fic domain (BepA - BepC $)^{15,66}$, with BepA being the best studied example. This protein has already been shown to possess AMPylation activity ${ }^{66}$. Unlike the proteins described above, the BepA host target is not known, but it does not appear to AMPylate Rho GTPases, nor does it cause cytoskeletal collapse.

In this study we set out to identify the host cell target proteins that the Fic domains of the Bartonella henselae BepA, BepB, BepC, as well as the B. quintana BepA1 and BepC proteins are acting on. Due to issues around solubility, this was limited to BepA and BepB from $B$. henselae. In order to accomplish this, fluorescent probes were utilised as labels to identify AMPylated host cell protein targets. These fluorescent probes are preferable to radioactive probes, which have been utilised in previous studies as they allow for easier detection, and don't require specialised disposal ${ }^{88,94,101}$. The fluorescent probes contain a FITC moiety that can be used to purify labelled proteins by immunoprecipitation with anti-FITC antibodies. This allows for purification and enrichment of the samples before running on an SDS-PAGE gel and excising for MS analysis if required. 
Another approach was to research related Bartonella proteins with reported Fic domain activity and known host cell protein targets. Currently, Bep2 is the only Bartonella effector studied that contains a Fic domain and that has a known host cell protein target. Similarities between Bep2 and the $B$. henselae Beps studied suggested they could have the same host cell target, vimentin. This formed the basis for the vimentin assays.

\subsection{Key Findings}

All experiments performed resulted in negative results. This included experiments using purified control proteins that had previously been shown to be active. Taken together, the results suggest that the purified proteins were enzymatically inactive. This is supported by the lack of host cell protein labelling using both F1-ATP and N6 pATP, despite using VopS, a positive control Fic domain protein that had previously been shown to AMPylate target protein in whole cell extract with both ATP analogues used here. This conclusion was also supported by lack of AMPylation activity detected in the pyrophosphate assay and LC-MS/MS analysis.

Finally, while both $B$. henselae and B. quintana BepC-Fic proteins and B. quintana BqBepA1 were successfully induced with IPTG, they could not be isolated during protein extraction.

\subsection{Future Directions}

\subsubsection{Improving Recombinant Protein Purification}

Before future experiments can be designed in order to determine the host cell protein targets of the $B$. henselae and $B$. quintana Fic domain-containing Bep proteins, the purification methods must be improved.

In these experiments, bacterial cell lysis was performed with B-PER reagent (Pierce). This system contains Tris- $\mathrm{HCl}(20 \mathrm{mM})$ and a proprietary, non-ionic, detergent to lyse the bacteria. While this reagent is described as mild, using it without additives could result in destabilised protein. One way that this problem could be avoided is by adding $\mathrm{NaCl}$ to the lysis buffer. High ionic strength is recommended in protein extraction buffer, as it enhances protein solubility and stability ${ }^{99}$. Previous extractions involving VopS, IbpA, and Bep2 have all been described with the addition of between $0.1-0.2 \mathrm{M} \mathrm{NaCl}^{75,79,84,88}$. In the case of $\mathrm{Bep} 2, \mathrm{MgCl}_{2}(10 \mathrm{mM})$ was also added to the lysis buffer. This could be helpful in this instance as $\mathrm{Mg}^{2+}$ has been described as a component of the active site for both VopS and BepA ${ }^{66,79}$. 
Similarly, many of the published lysis buffers for Fic-containing protein purification include either $\beta$-mercaptoethanol $(2.5-5 \mathrm{mM})^{84,88}$ or DTT $(1 \mathrm{mM})^{79,101}$ to prevent oxidation of the expressed proteins. Other additives to the lysis buffer could include DNase and lysozyme to aid in breakdown of nucleic acid and cell wall material to remove particularly viscous material before adding extracted protein to the nickel affinity columns ${ }^{99}$. Finally, addition of EDTA-free protease inhibitors could also be beneficial.

While the method we used has the benefit of being a simple and inexpensive method, it does involve the use of chemical additives to lyse the cell. In particular, Tris inhibits the click chemistry reaction by competing with the THPTA ligand for copper binding ${ }^{103}$. For these reasons, a buffer containing HEPES or phosphate is recommended. It is also not known what the detergent is in B-PER, or how it could be interacting with the expressed proteins. Previous studies have used NP-40 75 , or Triton X-100 as mild detergents. For these reasons, it is recommended in future protein extractions to use a lysis buffer containing $0.5-1 \%$ of either of these detergents, as well as the additives described.

Alternatively, mechanical lysis methods such as a French press or sonication could be utilised. Mechanical lysis has the benefit of avoiding chemicals that could potentially denature or inactive expressed proteins, but does require costlier equipment to perform. These methods have previously been used to purify several of the Bep and Fic-containing proteins, including Bep2 and $\operatorname{VopS}^{79,84}$.

\subsubsection{Protein Solubility}

As the $B$. henselae and B. quintana recombinant proteins BhBepC-Fic, BqBepA1, and BqBepCFic were insoluble and therefore not purified, a potential area to further explore are improvements in protein solubility.

\subsubsection{Altering Induction Conditions}

One of the most common methods to increase protein solubility is to decrease the temperature during the protein induction. Lower temperatures slow down all cell processes, resulting in lowered protein aggregation in inclusion bodies, as well as reduced activity of proteases ${ }^{95,98,99}$. This approach was attempted for BqBepA1, by inducing at $18^{\circ} \mathrm{C}$ for 24 hours without success; however, due to time constraints with the project, this was not attempted for either of the BepC-Fic proteins which may have more success. 
Another approach would be to reduce the concentration of IPTG used for induction. Several studies have observed increased solubility and activity using a lower IPTG concentration than recommended ${ }^{98}$. Previous studies of VopS, Bep2, BepA, IbpA, along with other Bep proteins all describe using between $0.05-0.5 \mathrm{mM}$ IPTG, with many recommending $0.4 \mathrm{mM}$, rather than $1 \mathrm{mM}$, as was used here $23,40,51,60,62,66,71,75,79,84,88,101$. Another factor to consider is the $\mathrm{pH}$ of the media, as fluctuation may denature proteins depending on their isoelectric points. To overcome this, a buffered media may be appropriate. Similarly, $\mathrm{pH}$ alteration of the media may also aid in solubilisation if the isoelectric point of the induced protein is particularly high or low ${ }^{95}$. Along these lines, an alternative media such as Terrific Broth (TB), could also aid in solubilisation. TB is a widely used media that is specifically formulated to increase protein solubility, as it contains both glycerol and phosphate buffer ${ }^{84,98,99}$. Alternatively, 2X YT media has also previously been described ${ }^{75,99}$. Supplementing the media with co-factors of the recombinant Fic proteins could also benefit solubility, as several proteins require co-factors to fold correctly ${ }^{95,99}$. Crystal structure studies with BepA and VopS have shown that $\mathrm{Mg}^{2+}$ ions appear to act as a co-factor in the binding site, and it is likely that the other Fic-containing proteins would also require magnesium ions ${ }^{66,79}$. While yeast extract does contain $\mathrm{Mg}^{2+}$, there is the possibility that increasing the amount of $\mathrm{Mg}^{2+}$ ions in the media could aid in protein solubility. Both TB and 2X YT media contain significantly more yeast extract, so these media should also be trialled in future experiments.

Other additives to the media include chemical chaperones such as sugars and amino acids that inhibit protein aggregation during refolding of misfolded or unfolded protein, can also be effective ${ }^{105}$. Some examples of useful sugars include sorbitol, a sugar alcohol formed by reduction of glucose, and trehalose, a disaccharide of glucose. For example, sorbitol has previously been shown to increase fluorescence of GFP 12 fold, an indication of increased solubility ${ }^{105}$.

Some examples of helpful amino acid additives include arginine and glycylglycine. The latter is a dipeptide of glycine that has previously been shown to increase solubility of PPE proteins by $170-$ fold $^{106}$ 
These strategies could also be applied to the enzymatically inactive recombinant proteins successfully purified in this study. While these proteins were soluble, it is possible that the inactivity observed was due to misfolding during protein induction. For this reason, many of these suggestions may also aid in purifying enzymatically active proteins. In particular, inductions should be trialled using a lower induction temperature, using an alternative media such as TB, and including additives that aid in protein folding such as sorbitol, arginine, or additional $\mathrm{MgCl}_{2}$ in the media.

\subsubsection{Fusion Tags}

If these strategies were not able to increase protein solubility, alternative fusion tags could be employed, although this would require creating new expression constructs. There are several fusion tags that can improve protein solubility or promote folding, some of which are described below.

The two most common fusion tags that enhance solubility are thioredoxin (Trx) and maltosebinding protein (MBP). Trx aids in both solubilisation of proteins and in disulfide bond formation, although it is not sufficient for protein purification without a second $\operatorname{tag}^{98}$. Trx also typically works best at the $\mathrm{N}$-terminus, so if a His 6 tag were to be used in purification, it would need to be C-terminal. MBP is an alternative fusion tag that has the benefit of passively promoting folding of its fused protein partner ${ }^{98}$. MBP also has the benefit of use as a selection agent during purification, as it can bind sugars bound to agarose or sepharose beads ${ }^{98}$. The downside to MBP is that it is a large protein $(42 \mathrm{kDa})$ and this size is more likely to interfere with protein activity. For this reason MBP is usually cleaved before assays are performed. It can also solubilise misfolded proteins, resulting in time wasted on purifying inactive proteins $^{98,99}$.

Other fusion tags include $\mathrm{N}$-utilisation substance $\mathrm{A}$ (NusA), a transcription termination/antitermination factor that was shown to be effective at solubilising proteins ${ }^{98}$. However, NusA is a large protein $(55 \mathrm{kDa})$ and requires a purification tag. Solubility enhancing tags (SETs) consist of small, highly acidic peptides and help promote soluble expression of folded proteins that are prone to aggregation could also be utilised ${ }^{98}$. The main benefit of these tags is their small size, typically $<40$ amino acids. 
Finally the small ubiquitin like modifier (SUMO) tag, is small (11.2 kDa) and has a specific protease for cleavage, therefore avoiding the risk of cleavage of target protein ${ }^{98}$. The only downside is it must be $\mathrm{N}$-terminal and can't precede a proline moiety.

It is worth noting that most fusion tags benefit solubility by being in the $\mathrm{N}$-terminal position. $\mathrm{N}$-terminal tags also have the benefit of exposing the ribosome to an initiator sequence that is suitable for $E$. coli99. If a second purification tag, such as His 6 or GST, is required, it is recommended that it be at the $\mathrm{C}$-terminus position. In particular, the $\mathrm{His} 6$ tag can benefit from being C-terminal as when purification occurs, only fully translated proteins will be purified.

\subsubsection{Molecular Chaperones}

Finally, co-expression with molecular chaperones could enhance protein solubility by aiding in protein folding ${ }^{95,98,99}$. For example both the DnaK-DnaJ-GrpE and GroEL-GroES systems bind-solvent exposed hydrophobic domains, preventing peptide aggregation ${ }^{98}$. One study has shown that by overexpressing DnaK-DnaJ-GrpE, either by a plasmid system, or by adding 3\% $\mathrm{v} / \mathrm{v}$ ethanol to the media, the solubility of preS2-S'- $\beta$-galactosidase was increased 2 -fold ${ }^{107}$. Most recombinant proteins favour one of these two chaperone systems, so it is best to test each system separately. The downside to using one of these two chaperone systems however, is that they have reduced activity when cultured at lower temperatures. When lower temperatures are desired, the cold-adapted Cpn10-Cpn60 system, can be utilised instead ${ }^{98}$.

When using molecular chaperones, it is important to overexpress them before expressing the recombinant protein, as insoluble proteins are unable to re-fold once in inclusion bodies ${ }^{107}$.

\subsubsection{Follow-up Experiments}

As discussed, the click chemistry assay appears to work in terms of coupling the azide and alkyne, but future efforts must be concentrated on expressing soluble, active proteins. This is an essential prerequisite for subsequent experiments, such as whole cell extract labelling, click chemistry experiments, or mass spectrometry analysis of potential targets. If purification of labelled proteins is required before mass spectrometry analysis, an alternative azide tag can also be utilised along with the 6-FAM-azide. For example, Grammel et al. (2011) utilised a biotin tagged azide for mass spectrometry analysis. This allowed for purification of labelled protein on streptavidin columns. 
There are several biotin-tagged azide derivatives available, and this method could be used in future experiments over anti-FITC antibodies, which could have issues with non-specific binding.

Alternatively, before click chemistry experiments are undertaken, both recombinant vimentin and Cdc42 could be used in pyrophosphate assays and mass spectrometry analysis to test protein purification methodology for enzymatically active recombinant Fic proteins. Similarly, if BrBep2-Fic can be shown to have activity during whole cell extract labelling, then vimentin polymerisation assays could also be revisited with this enzyme, as well as SpyA from $S$. pyogenes to act as a positive control ${ }^{89}$.

If these experiments were to successfully identify target protein, additional experiments could also be performed.

\subsubsection{Host Cell Protein Analysis}

Analysis of any identified AMPylated host cell proteins could be undertaken. This could include changes in expression or localisation in the presence and absence of recombinant Fic protein. For proteins of known function, changes in activity could be further studied, such as how downstream effectors of the protein may be affected. The effects of target protein modification on host cell morphology and integrity could also be analysed. This could involve fluorescence microscopy techniques, to identify changes to host cell morphology, as well as analysis of markers that indicate cellular health.

During this project, both BhBepA-Fic and BhBepA_H159A-Fic were cloned into a eukaryotic expression system, with a GFP C-terminal fusion tag, for transfection and expression in cultured human cells. However, due to time constraints, these plasmids were not further developed. With this system, analysis of wound repair, intracellular localisation of Beps using fluorescence microscopy, as well as staining for effects on potential intracellular protein targets, could be looked at. Similarly, changes in mRNA expression could also be analysed to determine the effect of BhBepA-Fic expression on host cell protein expression. Similar constructs could also be made for the other Beps studied if required. 


\subsubsection{Fic Activity Inhibitors}

Future work could also look at inhibitors of the recombinant Bep proteins. This could involve creating an assay to screen a compound library for potential molecules that inhibit the activity of the Fic domain in these proteins. Those identified in an initial screen could be tested under a number of different assays such as an internalisation assay for Bartonella henselae Houston1 strain. If such an inhibitor was effective this could be further characterised as a potential Bartonella-specific antibiotic. Similar work has already been investigated for VopS. Lewallen et al. (2014) have described an assay using F1-ATP and Cdc42 to test for inhibition of VopS. This assay resulted in identification of several compounds with moderate efficacy in inhibiting VopS AMPylation of $\mathrm{Cdc42} 2^{108}$. This suggests that a similar assay could be developed for the recombinant Fic proteins studied here. 


\section{Concluding Remarks}

This work built on previous studies investigating novel ATP analogues for successful labelling and identification of host cell protein targets in whole cell extract ${ }^{88,101}$. The key goal of this project was to identify novel targets of the $B$. henselae and $B$. quintana Fic domain-containing Bep proteins. While potential protein targets of BhBepA-Fic and BhBepB-Fic were not identified, significant progress was made in optimising whole cell extract recovery and labelling reaction conditions. This will provide a good starting point for future experiments once active Fic domain proteins can be expressed and purified. 


\section{Bibliography}

1. Jones KE, Patel NG, Levy MA, et al. 2008. Global trends in emerging infectious diseases. Nature 451: 990-993.

2. Karem KL, Paddock CD, Regnery RL. 2000. Bartonella henselae, B. quintana, and $B$. bacilliformis: historical pathogens of emerging significance. Microb Infect 2: 1193-1205.

3. Minnick MF, Anderson BE. 2015. Chapter 105 - Bartonella, 1911-1939. In Schwartzman Y-WTSLP (ed.), Molecular Medical Microbiology (Second Edition). Academic Press, Boston.

4. Maurin M, Raoult D. 1996. Bartonella (Rochalimaea) quintana infections. Clin Microbiol Rev 9: 273-292.

5. Breitschwerdt EB. 2014. Bartonellosis: One Health Perspectives for an Emerging Infectious Disease. ILAR J 55: 46-58.

6. Mogollon-Pasapera E, Otvos L, Jr., Giordano A, et al. 2009. Bartonella: emerging pathogen or emerging awareness? Int J Infect Dis 13: 3-8.

7. Relman DA, Loutit JS, Schmidt TM, et al. 1990. The Agent of Bacillary Angiomatosis. An Approach to the Identification of Uncultured Pathogens. N Engl J Med 323: 1573-1580.

8. Regnery RL, Anderson BE, Clarridge JE, et al. 1992. Characterization of a novel Rochalimaea species, $R$. henselae sp. nov., isolated from blood of a febrile, human immunodeficiency virus-positive patient. J Clin Microbio/ 30: 265-274.

9. Brenner DJ, O'Connor SP, Winkler HH, et al. 1993. Proposals To Unify the Genera Bartonella and Rochalimaea, with Descriptions of Bartonella quintana comb. nov., Bartonella vinsonii comb. nov., Bartonella henselae comb. nov., and Bartonella elizabethae comb. nov., and To Remove the Family Bartonellaceae from the Order Rickettsiales. Int J Syst Bacteriol 43: 777-786.

10. Kosoy M, Hayman DTS, Chan KS. 2012. Bartonella bacteria in nature: Where does population variability end and a species start? Infect Genet Evol 12: 894-904.

11. Maggi RG, Mascarelli PE, Pultorak EL, et al. 2011. Bartonella spp. bacteremia in high-risk immunocompetent patients. Diagn Microbiol Infect Dis 71: 430-437.

12. Rolain JM, Foucault C, Guieu R, et al. 2002. Bartonella quintana in human erythrocytes. Lancet 360: 226-228.

13. Maurin M, Birtles R, Raoult D. 1997. Current Knowledge of Bartonella Species. Eur J Clin Microbiol Infect Dis 16: 487-506.

14. Chomel BB, Kasten RW, Williams C, et al. 2009. Bartonella Endocarditis: A Pathology Shared by Animal Reservoirs and Patients. Ann N Y Acad Sci 1166: 120-126.

15. Harms A, Dehio C. 2012. Intruders below the Radar: Molecular Pathogenesis of Bartonella spp. Clin Microbiol Rev 25: 42-78.

16. Minnick MF, Anderson BE, Lima A, et al. 2014. Oroya Fever and Verruga Peruana: Bartonelloses Unique to South America. PloS Neglect Trop D 8: e2919.

17. Chomel BB, Boulouis H-J, Breitschwerdt EB, et al. 2009. Ecological fitness and strategies of adaptation of Bartonella species to their hosts and vectors. Vet Res 40: 29.

18. Giladi M, Maman E, Paran D, et al. 2005. Cat-Scratch Disease-Associated Arthropathy. Arthritis Rheum 52: 3611-3617.

19. Lantos PM, Maggi RG, Ferguson B, et al. 2014. Detection of Bartonella Species in the Blood of Veterinarians and Veterinary Technicians: A Newly Recognized Occupational Hazard? Vector Borne Zoonotic Dis 14: 563-570. 
20. Breitschwerdt EB, Maggi RG, Duncan AW, et al. 2007. Bartonella Species in Blood of Immunocompetent Persons with Animal and Arthropod Contact. Emerg Infect Dis 13: 938-941.

21. Maggi RG, Mozayeni BR, Pultorak EL, et al. 2012. Bartonella spp. Bacteremia and Rheumatic Symptoms in Patients from Lyme Disease-endemic Region. Emerg Infect Dis 18: 783-791.

22. Vayssier-Taussat M, Moutailler S, Femenia F, et al. 2016. Identification of Novel Zoonotic Activity of Bartonella spp., France. Emerg Infect Dis 22: 457-462.

23. Engel P, Goepfert A, Stanger FV, et al. 2012. Adenylylation control by intra- or intermolecular active-site obstruction in Fic proteins. Nature 482: 107-110.

24. Magalhães RF, Pitassi LHU, Salvadego M, et al. 2008. Bartonella henselae survives after the storage period of red blood cell units: is it transmissible by transfusion? Transfusion Med 18: 287-291.

25. Atenstaedt RL. 2006. Trench fever: the British medical response in the Great War. Journal of the Royal Society of Medicine 99: 564-568.

26. Drancourt M, Tran-Hung L, Courtin J, et al. 2005. Bartonella quintana in a 4000-Year-Old Human Tooth. J Infect Dis 191: 607-611.

27. Keefer CS, Greer W. 1950. Cat Scratch Fever. Trans Am Clin Climatol Assoc 62: 151-164.

28. Slater LN, Welch DF, Hensel D, et al. 1990. A Newly Recognized Fastidious GramNegative Pathogen as a Cause of Fever and Bacteremia. N Engl J Med 323: 1587-1593.

29. Joseph AK, Wood CW, Robson JM, et al. 2011. Bartonella henselae bacteraemia in domestic cats from Auckland. NZ Vet J 45: 185-187.

30. Kelly P, Rolain J-M, Raoult D. 2005. Prevalence of human pathogens in cat and dog fleas in New Zealand. NZ Med J 118.

31. Pitassi LH, Magalhaes RF, Barjas-Castro ML, et al. 2007. Bartonella henselae Infects Human Erythrocytes. Ultrastruct Patho/ 31: 369-372.

32. Mandle T, Einsele $H$, Schaller M, et al. 2005. Infection of human CD34+ progenitor cells with Bartonella henselae results in intraerythrocytic presence of $B$. henselae. Blood 106: 1215-1222.

33. Zarkovic A, McMurray C, Deva N, et al. 2007. Seropositivity rates for Bartonella henselae, Toxocara canis and Toxoplasma gondii in New Zealand blood donors. Clin Experiment Ophthalmol 35: 131-134.

34. Breitschwerdt EB, Maggi RG, Nicholson WL, et al. 2008. Bartonella sp. Bacteremia in Patients with Neurological and Neurocognitive Dysfunction. J Clin Microbio/ 46: 28562861.

35. Pitassi LHU, de Paiva Diniz PPV, Scorpio DG, et al. 2015. Bartonella spp. Bacteremia in Blood Donors from Campinas, Brazil. PLoS Negl Trop Dis 9: e0003467.

36. Breitschwerdt EB, Maggi RG, Sigmon B, et al. 2007. Isolation of Bartonella quintana from a Woman and a Cat following Putative Bite Transmission. J Clin Microbiol 45: 270-272.

37. Guy L, Nystedt B, Toft C, et al. 2013. A Gene Transfer Agent and a Dynamic Repertoire of Secretion Systems Hold the Keys to the Explosive Radiation of the Emerging Pathogen Bartonella. PLoS Genet 9: e1003393.

38. Engel $\mathrm{P}$, Salzburger W, Liesch $\mathrm{M}$, et al. 2011. Parallel Evolution of a Type IV Secretion System in Radiating Lineages of the Host-Restricted Bacterial Pathogen Bartonella. PLoS Genet 7: e1001296. 
39. Alsmark CM, Frank AC, Karlberg EO, et al. 2004. The louse-borne human pathogen Bartonella quintana is a genomic derivative of the zoonotic agent Bartonella henselae. Proc Natl Acad Sci U S A 101: 9716.

40. Okujava R, Guye P, Lu YY, et al. 2014. A Translocated Effector Required for Bartonella Dissemination from Derma to Blood Safeguards Migratory Host Cells from Damage by Co-Translocated Effectors. PLoS Pathog 10: e1004187.

41. Dehio C. 2001. Bartonella interactions with endothelial cells and erythrocytes. Trends Microbiol 9: 279-285.

42. Garcia FU, Wojta J, Broadley KN, et al. 1990. Bartonella bacilliformis Stimulates Endothelial Cells In Vitro and Is Angiogenic In Vivo. AM J Pathol 136: 1125-1135.

43. Garcia FU, Wojta J, Hoover RL. 1992. Interactions between Live Bartonella bacilliformis and Endothelial Cells. J Infect Dis 165: 1138-1141.

44. Verma A, Davis GE, Ihler GM. 2000. Infection of Human Endothelial Cells with Bartonella bacilliformis Is Dependent on Rho and Results in Activation of Rho. Infect Immun 68: 5960-5969.

45. Verma A, Davis GE, Ihler GM. 2001. Formation of stress fibres in human endothelial cells infected with Bartonella bacilliformis is associated with altered morphology, impaired migration and defects in cell morphogenesis. Cell Microbio/ 3: 169-169.

46. Pizarro-Cerdá J, Cossart P. 2006. Bacterial Adhesion and Entry into Host Cells. Cell 124: 715-727.

47. Walker TS. 1984. Rickettsial Interactions with Human Endothelial Cells In Vitro: Adherence and Entry. Infect Immun 44: 205-210.

48. Clerc P, Sansonetti PJ. 1987. Entry of Shigella flexneri into HeLa cells: Evidence for Directed Phagocytosis Involving Actin Polymerization and Myosin Accumulation. Infect Immun 55: 2681-2688.

49. Patel JC, Galan JE. 2005. Manipulation of the host actin cytoskeleton by Salmonella--all in the name of entry. Curr Opin Microbiol 8: 10-15.

50. Young VB, Falkow S, Schoolnik GK. 1992. The invasin protein of Yersinia enterocolitica: internalization of invasin-bearing bacteria by eukaryotic cells is associated with reorganization of the cytoskeleton. J Cell Biol 116: 197-207.

51. Truttmann MC, Rhomberg TA, Dehio C. 2011. Combined action of the type IV secretion effector proteins BepC and BepF promotes invasome formation of Bartonella henselae on endothelial and epithelial cells. Cell Microbiol 13: 284-299.

52. Truttmann MC, Misselwitz B, Huser S, et al. 2011. Bartonella henselae engages insideout and outside-in signaling by integrin $\beta 1$ and talin1 during invasome-mediated bacterial uptake. J Cell Sci 124: 3591-3602.

53. Franz B, Kempf VA. 2011. Adhesion and host cell modulation: critical pathogenicity determinants of Bartonella henselae. Parasit Vectors 4: 54.

54. Zhang P, Chomel BB, Schau MK, et al. 2004. A family of variably expressed outermembrane proteins (Vomp) mediates adhesion and autoaggregation in Bartonella quintana. Proc Natl Acad Sci U S A 101: 13630-13635.

55. Lu YY, Franz B, Truttmann MC, et al. 2013. Bartonella henselae trimeric autotransporter adhesin BadA expression interferes with effector translocation by the VirB/D4 type IV secretion system. Cell Microbiol 15: 759-778.

56. Vayssier-Taussat M, Le Rhun D, Deng HK, et al. 2010. The Trw Type IV Secretion System of Bartonella Mediates Host-Specific Adhesion to Erythrocytes. PLoS pathogens 6: e1000946. 
57. Deng HK, Le Rhun D, Le Naour E, et al. 2012. Identification of Bartonella Trw HostSpecific Receptor on Erythrocytes. PLoS One 7: e41447.

58. Seubert A, Hiestand R, de la Cruz F, et al. 2003. A bacterial conjugation machinery recruited for pathogenesis. Mol Microbiol 49: 1253-1266.

59. Schulein R, Dehio C. 2002. The VirB/VirD4 type IV secretion system of Bartonella is essential for establishing intraerythrocytic infection. Mol Microbiol 46: 1053-1067.

60. Schmid MC, Scheidegger F, Dehio M, et al. 2006. A Translocated Bacterial Protein Protects Vascular Endothelial Cells from Apoptosis. PLoS Pathog 2: e115.

61. Scheidegger F, Ellner Y, Guye P, et al. 2009. Distinct activities of Bartonella henselae type IV secretion effector proteins modulate capillary-like sprout formation. Cell Microbiol 11: 1088-1101.

62. Rhomberg TA, Truttmann MC, Guye P, et al. 2009. A translocated protein of Bartonella henselae interferes with endocytic uptake of individual bacteria and triggers uptake of large bacterial aggregates via the invasome. Cell Microbiol 11: 927-945.

63. Schmid MC, Schulein R, Dehio $M$, et al. 2004. The VirB type IV secretion system of Bartonella henselae mediates invasion, proinflammatory activation and antiapoptotic protection of endothelial cells. Mol Microbiol 52: 81-92.

64. Schröder G, Schuelein R, Quebatte M, et al. 2011. Conjugative DNA transfer into human cells by the VirB/VirD4 type IV secretion system of the bacterial pathogen Bartonella henselae. Proc Natl Acad Sci U S A 108: 14643-14648.

65. Schulein R, Guye P, Rhomberg TA, et al. 2005. A bipartite signal mediates the transfer of type IV secretion substrates of Bartonella henselae into human cells. Proc Natl Acad Sci U S A 102: 856-861.

66. Palanivelu DV, Goepfert A, Meury M, et al. 2011. Fic domain-catalyzed adenylylation: Insight provided by the structural analysis of the type IV secretion system effector BepA. Protein Sci 20: 492-499.

67. Hayashi T, Morohashi H, Hatakeyama M. 2013. Bacterial EPIYA effectors--Where do they come from? What are they? Where are they going? Cell Microbiol 15: 377-385.

68. Viboud GI, Bliska JB. 2005. Yersinia Outer Proteins: Role in Modulation of Host Cell Signaling Responses and Pathogenesis. Annu Rev Microbio/ 59: 69-89.

69. Mukherjee S, Keitany G, Li Y, et al. 2006. Yersinia YopJ Acetylates and Inhibits Kinase Activation by Blocking Phosphorylation. Science 312: 1211-1214.

70. Pulliainen AT, Pieles K, Brand CS, et al. 2012. Bacterial effector binds host cell adenylyl cyclase to potentiate Galphas-dependent cAMP production. Proc Natl Acad Sci U S A 109: 9581-9586.

71. Yarbrough ML, Li Y, Kinch LN, et al. 2009. AMPylation of Rho GTPases by Vibrio VopS Disrupts Effector Binding and Downstream Signaling. Science 323: 269-272.

72. Brown MS, Segal A, Stadtman ER. 1971. Modulation of Glutamine Synthetase Adenylylation and Deadenylylation Is Mediated by Metabolic Transformation of the P(II)-Regulatory Protein. Proc Natl Acad Sci U S A 68: 2949-2953.

73. Stadtman ER, Ginsburg A, Ciardi JE, et al. 1970. Multiple molecular forms of glutamine synthetase produced by enzyme catalyzed adenylation and deadenylylation reactions. Adv Enzyme Regul 8: 99-118.

74. Roy CR, Mukherjee S. 2009. Bacterial FIC Proteins AMP Up Infection. Sci Signal 2: pe14.

75. Worby CA, Mattoo S, Kruger RP, et al. 2009. The Fic domain: A new paradigm for adenylylation. Mol Cell 34: 93-103. 
76. Mukherjee S, Liu X, Arasaki K, et al. 2011. Modulation of Rab GTPase function by a protein phosphocholine transferase. Nature 477: 103-106.

77. Kinch LN, Yarbrough ML, Orth K, et al. 2009. Fido, a Novel AMPylation Domain Common to Fic, Doc, and AvrB. PLoS One 4: e5818.

78. Sanyal A, Chen AJ, Nakayasu ES, et al. 2015. A Novel Link between Fic (Filamentation Induced by cAMP)-mediated Adenylylation/AMPylation and the Unfolded Protein Response. J Biol Chem 290: 8482-8499.

79. Orth K, Luong PKLN, Grishin NV, et al. 2010. Kinetic and Structural Insights into the Mechanism of AMPylation by VopS Fic Domain. J Biol Chem 285: 20155-20163.

80. Dixon JE, Sankaran B, Worby CA, et al. 2010. Structural basis of Fic-mediated adenylylation. Nat Struct Mol Biol 17: 1004-1010.

81. Hayes F. 2003. Toxins-Antitoxins: Plasmid Maintenance, Programmed Cell Death, and Cell Cycle Arrest. Science 301: 1496-1499.

82. Unterholzner SJ, Poppenberger B, Rozhon W. 2013. Toxin-antitoxin systems: Biology, identification, and application. Mob Genet Elements 3: e26219.

83. Eremeeva ME, Gerns HL, Lydy SL, et al. 2007. Bacteremia, Fever, and Splenomegaly Caused by a Newly Recognized Bartonella Species. N Engl J Med 356: 2381-2387.

84. Pieles K, Glatter T, Harms A, et al. 2014. An experimental strategy for the identification of AMPylation targets from complex protein samples. Proteomics 14: 1048-1052.

85. Eriksson JE, Dechat T, Grin B, et al. 2009. Introducing intermediate filaments: from discovery to disease. J Clin Invest 119: 1763-1771.

86. Ausubel FM. 2001. Current Protocols in Molecular Biology. J. Wiley, New York.

87. Haendiges J, Timme R, Allard M, et al. 2014. Draft Genome Sequences of Clinical Vibrio parahaemolyticus Strains Isolated in Maryland (2010-2013). Genome Announc 2: e00776-00714.

88. Lewallen DM, Steckler CJ, Knuckley B, et al. 2012. Probing adenylation: using a fluorescently labelled ATP probe to directly label and immunoprecipitate VopS substrates. Mol Biosyst 8: 1701-1706.

89. Icenogle LM, Hengel SM, Coye LH, et al. 2012. Molecular and Biological Characterization of Streptococcal SpyA-mediated ADP-ribosylation of Intermediate Filament Protein Vimentin. J Biol Chem 287: 21481-21491.

90. Schiavo G, van der Goot FG. 2001. The bacterial toxin toolkit. Nat Rev Mol Cell Biol 2: 530-537.

91. Aktories K. 2011. Bacterial protein toxins that modify host regulatory GTPases. Nat Rev Microbiol 9: 487-498.

92. Galan JE. 2009. Common Themes in the Design and Function of Bacterial Effectors. Cell Host Microbe 5: 571-579.

93. Hao Y-H, Chuang T, Ball HL, et al. 2011. Characterization of a rabbit polyclonal antibody against threonine-AMPylation. J Biotechnol 151: 251-254.

94. Yu XB, Woolery AR, Luong P, et al. 2014. Copper-catalyzed azide-alkyne cycloaddition (click chemistry)-based Detection of Global Pathogen-host AMPylation on Selfassembled Human Protein Microarrays. Molecular \& Cellular Proteomics 13: 31643176.

95. Fakruddin M, Mohammad Mazumdar R, Bin Mannan KS, et al. 2013. Critical Factors Affecting the Success of Cloning, Expression, and Mass Production of Enzymes by Recombinant E. coli. ISRN Biotechnol 2013: 590587. 
96. Sharp PM, Li WH. 1987. The codon adaptation index--a measure of directional synonymous codon usage bias, and its potential applications. Nucleic Acids Res 15: 1281-1295.

97. Das S, Paul S, Chatterjee S, et al. 2005. Codon and Amino Acid Usage in Two Major Human Pathogens of Genus Bartonella--Optimization Between ReplicationalTranscriptional Selection, Translational Control and Cost Minimization. DNA Res 12: 91 102.

98. Francis DM, Page R. 2001. Strategies to Optimize Protein Expression in E. coli, Current Protocols in Protein Science. John Wiley \& Sons, Inc.

99. Structural Genomics C, China Structural Genomics C, Northeast Structural Genomics C, et al. 2008. Protein production and purification. Nat Methods 5: 135-146.

100. Hong V, Presolski SI, Ma C, et al. 2009. Analysis and Optimization of Copper-Catalyzed Azide-Alkyne Cycloaddition for Bioconjugation. Angew Chem Int Ed Eng/ 48: 9879-9883.

101. Grammel M, Luong P, Orth K, et al. 2011. A Chemical Reporter for Protein AMPylation. J Am Chem Soc 133: 17103-17105.

102. Broncel M, Serwa RA, Tate EW. 2012. A New Chemical Handle for Protein AMPylation at the Host-Pathogen Interface. Chembiochem 13: 183-185.

103. Presolski SI, Hong VP, Finn MG. 2011. Copper-Catalyzed Azide-Alkyne Click Chemistry for Bioconjugation. Curr Protoc Chem Biol 3: 153-162.

104. Simon NC, Aktories K, Barbieri JT. 2014. Novel bacterial ADP-ribosylating toxins: structure and function. Nat Rev Microbiol 12: 599-611.

105. Prasad S, Khadatare PB, Roy I. 2011. Effect of Chemical Chaperones in Improving the Solubility of Recombinant Proteins in Escherichia coli. Appl Environ Microbiol 77: 46034609.

106. Ghosh S, Rasheedi S, Rahim SS, et al. 2004. Method for enhancing solubility of the expressed recombinant proteins in Escherichia coli. Biotechniques 37: 418-423.

107. Thomas JG, Baneyx F. 1996. Protein Misfolding and Inclusion Body Formation in Recombinant Escherichia coli Cells Overexpressing Heat-Shock Proteins. J Biol Chem 271: 11141-11147.

108. Lewallen DM, Sreelatha A, Dharmarajan V, et al. 2014. Inhibiting AMPylation: A Novel Screen To Identify the First Small Molecule Inhibitors of Protein AMPylation. ACS chemical biology 9: 433-442. 


\section{Appendix}

\subsection{Sequence Alignments}

The following sequence alignments were performed using the multiple sequence alignment tool ClustalOmega (Goujon et al., 2010; Sievers et al., 2011) (available online at www.ebi.ac.uk/Tools/msa/clustalo/ - 23/5/16).

\subsubsection{Bartonella henselae BepA-Fic H159A mutant design}

BhBepA Fic fragment BhBepAFicH159A_40 consensus

BhBepA Fic fragment BhBepAFicH159A_40 consensus

BhBepA Fic fragment BhBepAFicH159A_40

consensus

BhBepA Fic fragment BhBepAFicH159A_40

consensus

BhBepA Fic fragment BhBepAFicH159A_40

consensus

BhBepA Fic fragment BhBepAFicH159A 40

consensus

BhBepA Fic fragment BhBepAFicH159A_40

consensus

BhBepA Fic fragment BhBepAFicH159A_40 consensus

BhBepA Fic fragment BhBepAFicH159A_40 consensus

BhBepA Fic fragment BhBepAFicH159A_40 consensus

BhBepA Fic fragment BhBepAFicH159A_40 consensus

BhBepA Fic fragment BhBepAFicH159A_40 consensus
ATGCCAAAGGCAAAAGCAAAAACGAAAAATACAGAAATTATTTCCCCCCATCATTAT ATGCCAAAGGCAAAAGCAAAAACGAAAAATACAGAAATTATTTCCCCCCATCATTAT

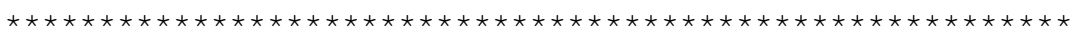
GTCTATCCTAACACCACAACACTCAAAAATAAATATGGAATAAAAAACTTAAATGCT GTCTATCCTAACACCACAACACTCAAAAATAAATATGGAATAAAAAACTTAAATGCT

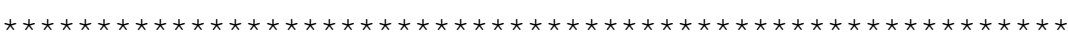

TTTCTGGAAAAATGCTCGCATGATACAGCCAAAGCAATGATCAATCTGCGTGAAGAA TTTCTGGAAAAATGCTCGCATGATACAGCCAAAGCAATGATCAATCTGCGTGAAGAA $* * * * * * * * * * * * * * * * * * * * * * * * * * * * * * * * * * * * * * * * * * * * * * * * * * * * * * * * *$

TCCTTACCAGAATATTTTGATACCGCCTATCTATGCCATATTCATCAACAGTTGTTT TCCTTACCAGAATATTTTGATACCGCCTATCTATGCCATATTCATCAACAGTTGTTT $* * * * * * * * * * * * * * * * * * * * * * * * * * * * * * * * * * * * * * * * * * * * * * * * * * * * * * * * *$

AAAAATACATTTGAATGGGCTGGATACCTTCGCCATATCCCCTTCACATTTGCAGAT AAAAATACATTTGAATGGGCTGGATACCTTCGCCATATCCCCTTCACATTTGCAGAT

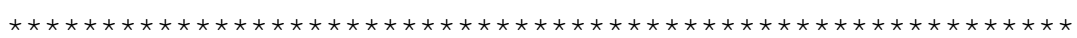

GGCACCACAGCCGCCATGCCAGAAATGAAAAGAACGGGTTGGAAAAATGCTTTTGCA GGCACCACAGCCGCCATGCCAGAAATGAAAAGAACGGGTTGGAAAAATGCTTTTGCA

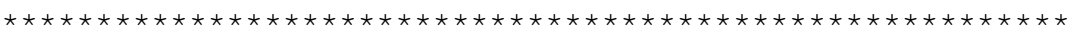

ATTGGTGATGAGATCCAAGAAGGCTTGCAAAGATTAGATCAAACACTTGCCGAAAAA ATTGGTGATGAGATCCAAGAAGGCTTGCAAAGATTAGATCAAACACTTGCCGAAAAA

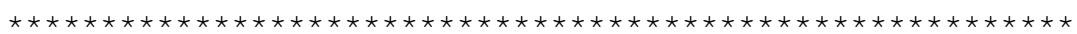

AACAATTTACAAGGTTTAACGCGCGAAGAATTTAACTCTGAAGCAATAGAACTGTTT AACAATTTACAAGGTTTAACGCGCGAAGAATTTAACTCTGAAGCAATAGAACTGTTT $* * * * * * * * * * * * * * * * * * * * * * * * * * * * * * * * * * * * * * * * * * * * * * * * * * * * * * * * *$

AATTCTCTCAACCAACTCCACCCATTCAGAGAAGGGAATGGACGAACACAACGGCTA AATTCTCTCAACCAACTCGCCCCATTCAGAGAAGGGAATGGACGAACACAACGGCTA

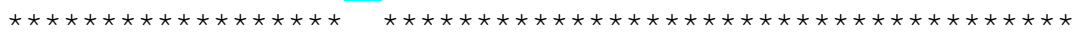

TTTTTTGAAAATCTTGCCAAAGCAGCAGGACATCAGCTTAACTTCTCGCTTATCACA TTTTTTGAAAATCTTGCCAAAGCAGCAGGACATCAGCTTAACTTCTCGCTTATCACA

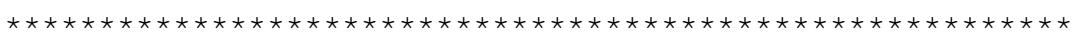

AAAGAACGCATGATGGTTGCCAGTGTTGCGGTAGCAGAAAATGGTGACCTAGAACCT AAAGAACGCATGATGGTTGCCAGTGTTGCGGTAGCAGAAAATGGTGACCTAGAACCT

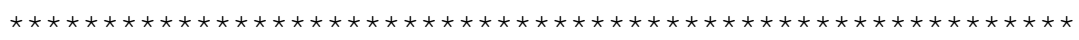

ATGCAACATTTGTTTGAgGATATCTCCAATCCAGAAAAAATTCGTCTTTTAAAAGAA ATGCAACATTTGTTTGAGGATATCTCCAATCCAGAAAAAATTCGTCTTTTAAAAGAA

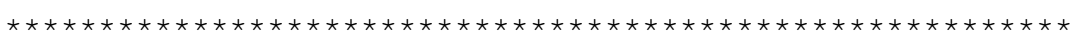




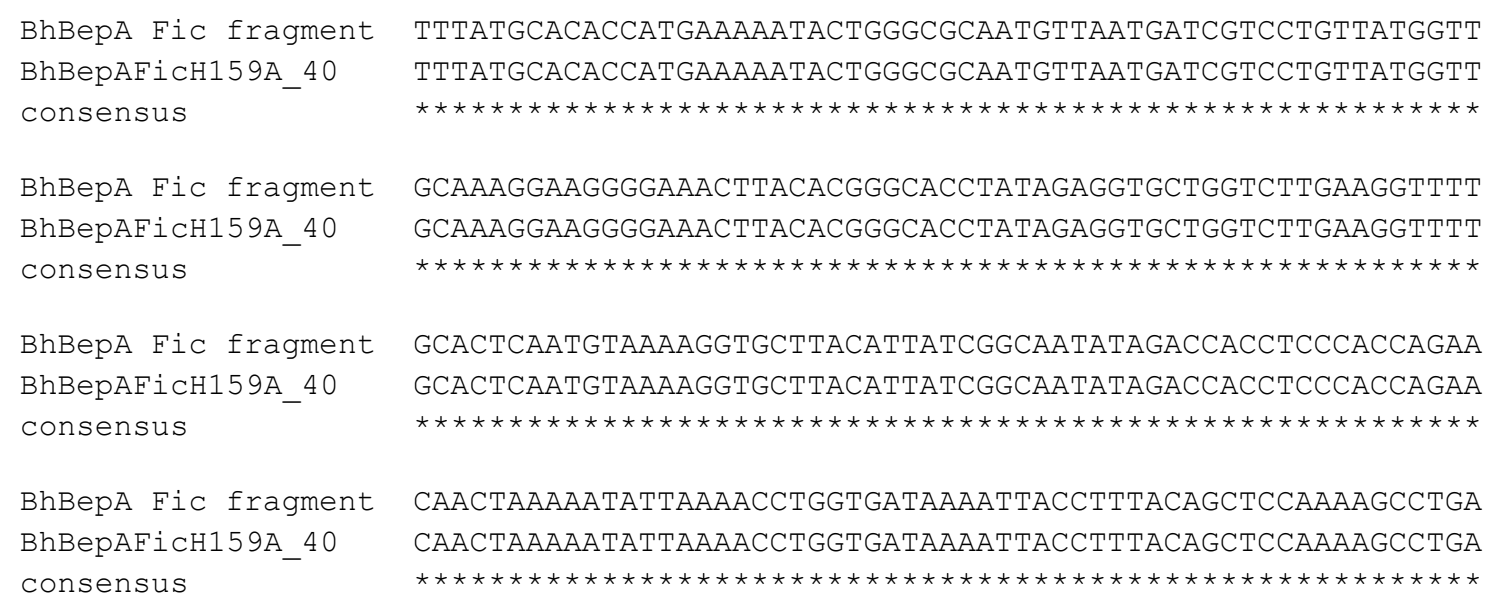

Appendix Figure 9.1: BhBepA-Fic H159A mutant sequence alignments. BepA sequence data was obtained from NCBI for $B$. henselae Houston-1 strain for the gene fragment expressed in this study and the additional stop codon. Blue indicates base changes introduced in the H159A Fic domain catalytic mutant 


\subsubsection{B. henselae BepB-Fic Alignment}

pET28a+BepBFic_insert BhBepBFic_19

pET28a+BepBFic_insert BhBepBFic_19

pET28a+BepBFic_insert BhBepBFic_19

pET28a+BepBFic_insert BhBepBFic_19

pET28a+BepBFic_insert BhBepBFic_19

pET28a+BepBFic_insert BhBepBFic_19

pET28a+BepBFic_insert BhBepBFic_19

pET28a+BepBFic insert BhBepBFic_19

pET28a+BepBFic_insert BhBepBFic_19

pET28a+BepBFic_insert BhBepBFic_19

pET28a+BepBFic_insert BhBepBFic_19

pET28a+BepBFic_insert BhBepBFic_19

pET28a+BepBFic insert BhBepBFic_19

pET28a+BepBFic_insert BhBepBFic_19
GACTCACTATAGGGGAATTGTGAGCGGATAACAATTCCCCTCTAGAAATAATTTTGTTTA GACTCACTATAGGGGAATTGTGAGCGGATAACAATTCCCCTCTAGAAATAATTTTGTTTA

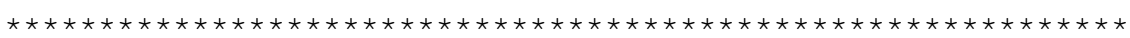

ACTTTAAGAAGGAGATATACCATGGGCAGCAGCCATCATCATCATCATCACAGCAGCGGC ACTTTAAGAAGGAGATATACCATGGGCAGCAGCCATCATCATCATCATCACAGCAGCGGC

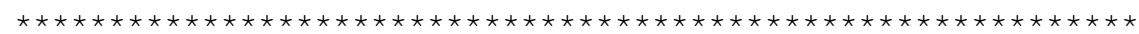

CTGGTGCCGCGCGGCAGCCATATGCCAAAAGCAAAAGCAAAAAATATCTCCACCGCTTCT CTGGTGCCGCGCGGCAGCCATATGCCAAAAGCAAAAGCAAAAAATATCTCCACCGCTTCT

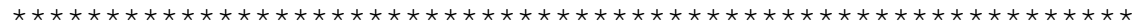

CCCCATAATTATATCTATCCTGGTACCCAAATACTCAAAAATAAATATGGAGAAACAGAT CCCCATAATTATATCTATCCTGGTACCCAAATACTCAAAAATAAATATGGAGAAACAGAT

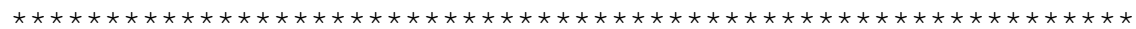

TTAAAACTCCTTCTGGAAAAATGCTTACATGATAGGGAACAAGCAATGATGAATCTGCGT TTAAAACTCCTTCTGGAAAAATGCTTACATGATAGGGAACAAGCAATGATGAATCTGCGT $* * * * * * * * * * * * * * * * * * * * * * * * * * * * * * * * * * * * * * * * * * * * * * * * * * * * * * * * * * * *$

GCAGAATCCCTACCAGAATATTTTGATTGCGCTTATCTATGCTATATCCACCAACAATTG GCAGAATCCCTACCAGAATATTTTGATTGCGCTTATCTATGCTATATCCACCAACAATTG

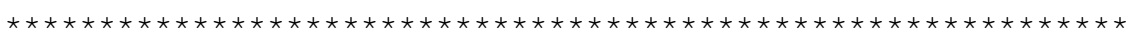

TTTCAAAAAACCTTTGAATGGGCTGGCCATCTCCGCCACACTCCCTTCACATTTGCAGAT TTTCAAAAAACCTTTGAATGGGCTGGCCATCTCCGCCACACTCCCTTCACATTTGCAGAT

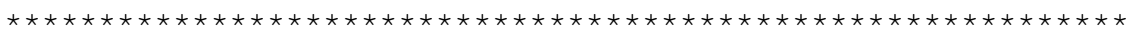

GGTAGCATTGCCGCCATGCCAGAAATGAAAAGAACAGAATGGGGCAAAGATTTTGCAAAA GGTAGCATTGCCGCCATGCCAGAAATGAAAAGAACAGAATGGGGCAAAGATTTTGCAAAA

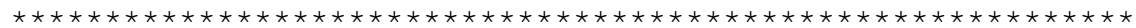

AGTGAAAAAATCCCCGAACTTTTACAAAAATTAGATCAAGAGCTTGCCGAAAAAGACAAT AGTGAAAAAATCCCCGAACTTTTACAAAAATTAGATCAAGAGCTTGCCGAAAAAGACAAT

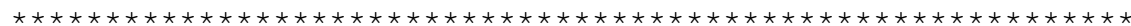

TTGCAAGGCTTAACACGCGAAGAGTTTATCAAGCAAGCAACGGAGCTATTTTATTCTCTT TTGCAAGGCTTAACACGCGAAGAGTTTATCAAGCAAGCAACGGAGCTATTTTATTCTCTT

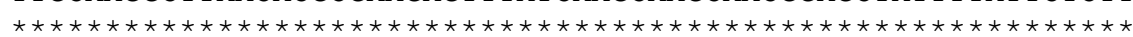

CACAAAATCCACCCATTCATAGATGGCAATGAACACACAGAACAATTCTTTTTTGAAAAT CACAAAATCCACCCATTCATAGATGGCAATGAACACACAGAACAATTCTTTTTTGAAAAT

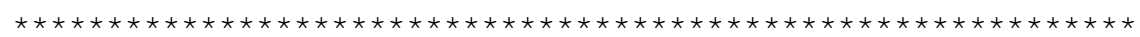

CTCGCCAAAGCCGCCGGACATCAACTTGACTTTTCGCTTGTCACACAAAAACGCATGATG CTCGCCAAAGCCGCCGGACATCAACTTGACTTTTCGCTTGTCACACAAAAACGCATGATG

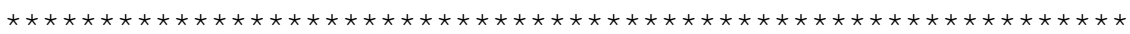

GCAGTCTGTAGCGAAGCAATGCAATATGGCGATACACAACTGATGAAAGATCTTTTTGAA GCAGTCTGTAGCGAAGCAATGCAATATGGCGATACACAACTGATGAAAGATCTTTTTGAA

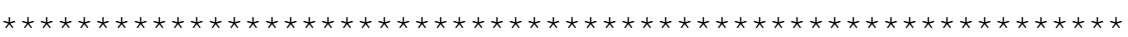

GATATCTCCAATCCAGAAAAAATACGTCTTTTAAAAGACTTTATGAACAACATCAACAAT GATATCTCCAATCCAGAAAAAATACGTCTTTTAAAAGACTTTATGAACAACATCAACAAT






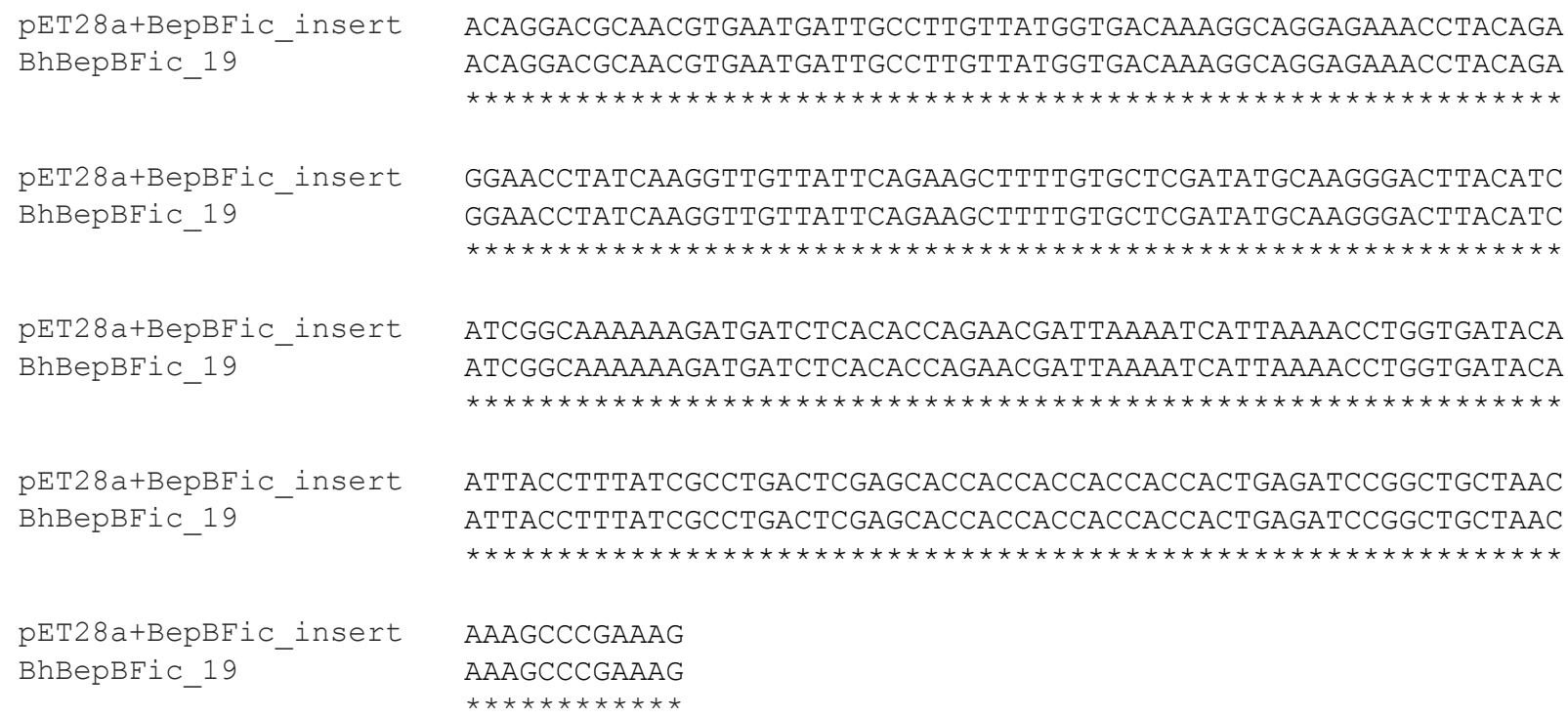

Appendix Figure 9.2: BhBepB-Fic sequence alignment. pET28a sequence data was obtained from Invitrogen, BepB sequence data was obtained from NCBI for B. henselae Houston-1 strain. pET28a insert sequence was generated by inserting the target BepB sequence between the Ndel and Xhol cut sites, along with an additional stop codon preceding the Xhol cut site. 


\subsubsection{B. henselae BepC Alignment}

pET28a+BhBepCFic_insert TAGGGGAATTGTGAGCGGATAACAATTCCCCTCTAGAAATAATTTTGTTTAACTTTAAGA BhBepCFic_10 TAGGGGAATTGTGAGCGGATAACAATTCCCCTCTAGAAATAATTTTGTTTAACTTTAAGA

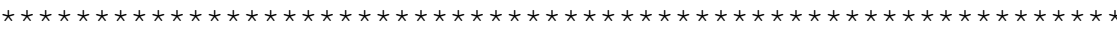

pET28a+BhBepCFic_insert BhBepCFic_10

pET28a+BhBepCFic_insert BhBepCFic_10

pET28a+BhBepCFic_insert BhBepCFic_10

pET28a+BhBepCFic_insert BhBepCFic_10

pET28a+BhBepCFic_insert BhBepCFic_10

pET28a+BhBepCFic_insert BhBepCFic_10

pET28a+BhBepCFic_insert BhBepCFic_10

pET28a+BhBepCFic_insert BhBepCFic_10

pET28a+BhBepCFic_insert BhBepCFic_10

pET28a+BhBepCFic_insert BhBepCFic_10

pET28a+BhBepCFic_insert BhBepCFic_10

pET28a+BhBepCFic_insert BhBepCFic_10

pET28a+BhBepCFic_insert BhBepCFic_10
AGGAGATATACCATGGGCAGCAGCCATCATCATCATCATCACAGCAGCGGCCTGGTGCCG AGGAGATATACCATGGGCAGCAGCCATCATCATCATCATCACAGCAGCGGCCTGGTGCCG $\star * * * * * * * * * * * * * * * * * * * * * * * * * * * * * * * * * * * * * * * * * * * * * * * * * * * * * * * * * * *$

CGCGGCAGCCATATGTTAGAGCATAATTATCTGTATAAAAACAGCAACACACTGAAAAAT CGCGGCAGCCATATGTTAGAGCATAATTATCTGTATAAAAACAGCAACACACTGAAAAAT

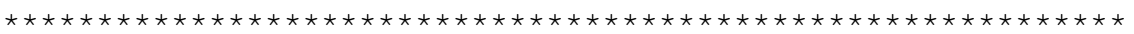

AAATATGGCATAAAAGACCCGCAAAGACTGTATGAGCGCTGTGCCCATGATACAGCCAGA AAATATGGCATAAAAGACCCGCAAAGACTGTATGAGCGCTGTGCCCATGATACAGCCAGA



GAGGTTATGAATCTTCGCTTTGAACCACCCCCACAAAGATTTGGTCTCGCTTATTTGAAA GAGGTTATGAATCTTCGCTTTGAACCACCCCCACAAAGATTTGGTCTCGCTTATTTGAAA

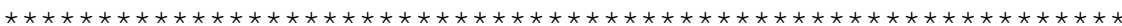

TTGATCCATTGGACCCTCTTCCATAGGAGTTTTGAATGGGCGGGTCATACTCGTGATGAG TTGATCCATTGGACCCTCTTCCATAGGAGTTTTGAATGGGCGGGTCATACTCGTGATGAG

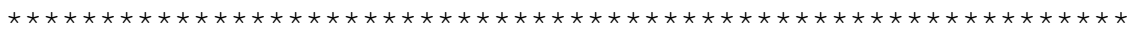

CCCTTTACATTTGAAGATGGTAGCACCGCCCGTATGCCAGCTATGCGCCCAAAAGGTCAT CCCTTTACATTTGAAGATGGTAGCACCGCCCGTATGCCAGCTATGCGCCCAAAAGGTCAT

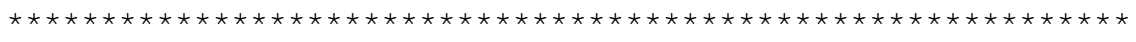

AGGGTTCCTTTTGCCGTTGGTCCACAGATTCAAAAAGAACTTAAACAATTAGAGAGAAAA AGGGTTCCTTTTGCCGTTGGTCCACAGATTCAAAAAGAACTTAAACAATTAGAGAGAAAA

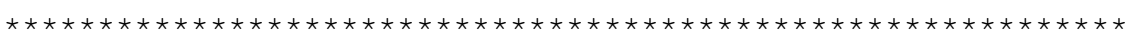

CTTACCGCAAAGAATAACTTACAAGGCTTATCACGCCAAGAGTTTGCTGAAAGTGCTGCG CTTACCGCAAAGAATAACTTACAAGGCTTATCACGCCAAGAGTTTGCTGAAAGTGCTGCG

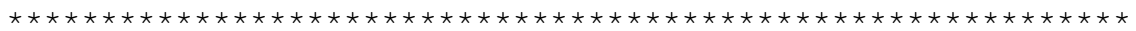

GAAGTTTTTATGACTCTCGACCACGCGCATCCTTTCAGAAAAGGCAATGGGCGCGCGCAA GAAGTTTTTATGACTCTCGACCACGCGCATCCTTTCAGAAAAGGCAATGGGCGCGCGCAA

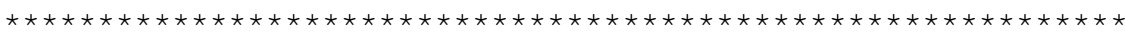

AGAATATTTATGGAAAAGCTTGGACAAGCAGCAGGCTATAAGATTGACTTTTCTTTTATC AGAATATTTATGGAAAAGCTTGGACAAGCAGCAGGCTATAAGATTGACTTTTCTTTTATC

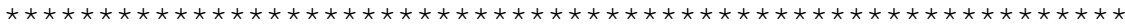

ACAAAAGAGCGCCTCACCCAAGCTAGTATTGCCGCAATGCAACATGGCAACACACAACCC ACAAAAGAGCGCCTCACCCAAGCTAGTATTGCCGCAATGCAACATGGCAACACACAACCC

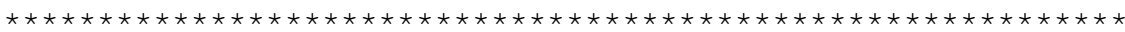

ATGAAAGATCTTTTTGAGGATATCACCCACCCGCAAAAATTCCTTCTTTTAAAGGAATTC ATGAAAGATCTTTTTGAGGATATCACCCACCCGCAAAAATTCCTTCTTTTAAAGGAATTC

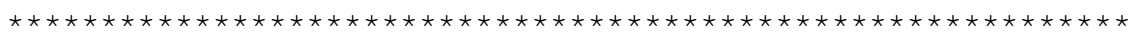

ATCTCTCAAATGAGAAATAGCGGACTTGAGGAAATCAACGAACATATTGTTGTCGTAGCA ATCTCTCAAATGAGAAATAGCGGACTTGAGGAAATCAACGAACATATTGTTGTCGTAGCA

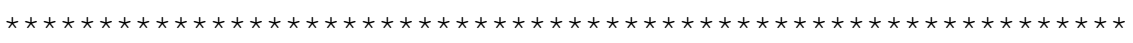


pET28a+BhBepCFic_insert
BhBepCFic_10

pET28a+BhBepCFic_insert BhBepCFic_10

pET28a+BhBepCFic_insert BhBepCFic_ 10

pET28a+BhBepCFic_insert BhBepCFic 10

pET28a+BhBepCFic_insert BhBepCFic_10
AAAGAAGGTGTAAAGTATGATGGCATCTATAAAGGTTGTGCTGCAGAAGGTTTTGTCATA AAAGAAGGTGTAAAGTATGATGGCATCTATAAAGGTTGTGCTGCAGAAGGTTTTGTCATA

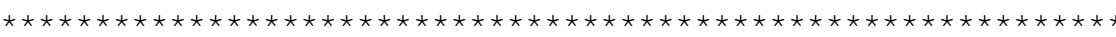

GAATTAGAAGATGGTTTTGTTGTCGGCCACAAAGATGATCTTACGCCAGAACAAGTCAAA GAATTAGAAGATGGTTTTGTTGTCGGCCACAAAGATGATCTTACGCCAGAACAAGTCAAA

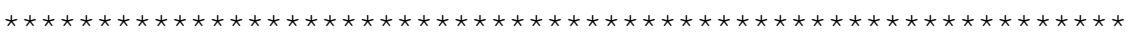

ACATTACAGAACGGTGATCGCATAGTCTGACTCGAGCACCACCACCACCACCACTGAGAT ACATTACAGAACGGTGATCGCATAGTCTGACTCGAGCACCACCACCACCACCACTGAGAT

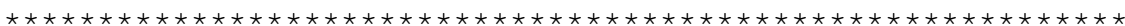

CCGGCTGCTAACAAAGCCCGAAAGGAAGCTGAGTTGGCTGCTGCCACCGCTGAGCAATAA CCGGCTGCTAACAAAGCCCGAAAGGAAGCTGAGTTGGCTGCTGCCACCGCTGAGCAATAA

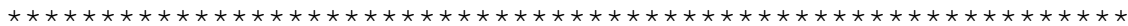

CTAGCATAACCCCTTGGGGCCTCTAAACG CTAGCATAACCCCTTGGGGCCTCTAAACG Сt*

Appendix Figure 9.3: Bh BepC-Fic sequence alignment. pET28a sequence data was obtained from Invitrogen, BepC sequence data was obtained from NCBI for B. henselae Houston-1 strain. pET28a insert sequence was generated by inserting the target $\mathrm{BepC}$ sequence between the Ndel and Xhol cut sites, along with an additional stop preceding the Xhol cut site. 


\subsubsection{B. quintana BepA1 Alignment}

pET28a+BqBepA1_insert BqBepA1_1

pET28a+BqBepA1_insert BqBepA1_1

pET28a+BqBepA1_insert BqBepA1_1

pET28a+BqBepA1_insert BqBepA1_1

pET28a+BqBepA1_insert BqBepA1_1

pET28a+BqBepA1_insert BqBepA1_1

pET28a+BqBepA1_insert BqBepA1_1

pET28a+BqBepA1_insert BqBepA1_1

pET28a+BqBepA1_insert BqBepA1_1

pET28a+BqBepA1_insert BqBepA1_1

pET28a+BqBepA1_insert BqBepA1_1

pET28a+BqBepA1_insert BqBepA1_1

pET28a+BqBepA1_insert BqBepA1_1
ATACGACTCACTATAGGGGAATTGTGAGCGGATAACAATTCCCCTCTAGAAATAATTTTG ATACGACTCACTATAGGGGAATTGTGAGCGGATAACAATTCCCCTCTAGAAATAATTTTG

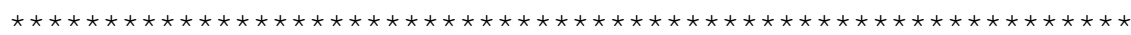

TTTAACTTTAAGAAGGAGATATACCATGGGCAGCAGCCATCATCATCATCATCACAGCAG TTTAACTTTAAGAAGGAGATATACCATGGGCAGCAGCCATCATCATCATCATCACAGCAG

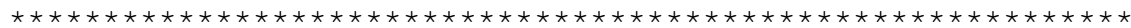

CGGCCTGGTGCCGCGCGGCAGCCATATGCCAAAAGCAAAGTCAAAAGAAACGCAAACACC CGGCCTGGTGCCGCGCGGCAGCCATATGCCAAAAGCAAAGTCAAAAGAAACGCAAACACC

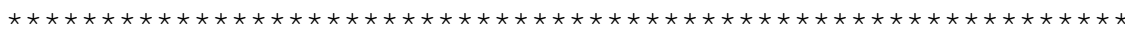

ATCCTCTACCCGCACCTCCTTACCTTATCATTATCTTTATTCTGATAGCAACATCCTGAA ATCCTCTACCCGCACCTCCTTACCTTATCATTATCTTTATTCTGATAGCAACATCCTGAA

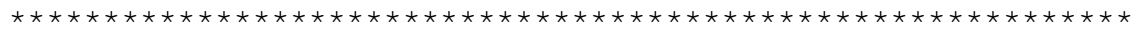

AAATAAATATAGAATAAAAAATTTGGATGTTTTTCTTAAGAAATGTTCGCATGATACGAC AAATAAATATAGAATAAAAAATTGGATGTTTTTCTTAAGAAATGTTCGCATGATACGAC

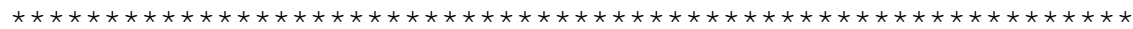

AAAAGCAATGGTCAATCTGTGCCAAGCATCCTTGCCAGAAAAGCTTGATTCTTCCTATCT AAAAGCAATGGTCAATCTGTGCCAAGCATCCTTGCCAGAAAAGCTTGATTCTTCCTATCT

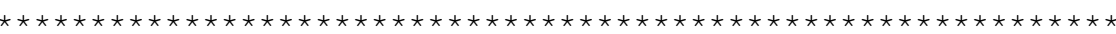

AAgAgATCTTCATCAACAgTTATTTCAAAATACGTTTGAATGGGCTGAAACTACCCGTGA AAGAGATCTTCATCAACAGTTATTTCAAAATACGTTTGAATGGGCTGAAACTACCCGTGA

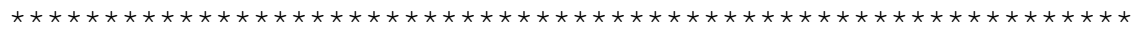

TACAGCCTTTAAATTTGAAGATGGCACCACTGCTGTCATGCCAGAAATGAAAAGAACAGG TACAGCCTTTAAATTTGAAGATGGCACCACTGCTGTCATGCCAGAAATGAAAAGAACAGG

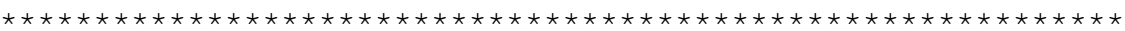

GTGGAAAAATCCCTTTTCAATCGATGATGAAATCCAAAAAGGCTTACAAAAATTAGATCG GTGGAAAAATCCCTTTTCAATCGATGATGAAATCCAAAAAGGCTTACAAAAATTAGATCG

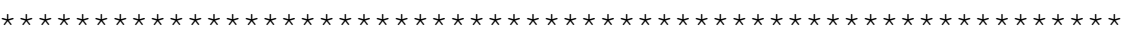

CACACTTGCTGAAAAAAACAATTTGCAAGGCTTATCACGTGAAGCGTTTATCTGTGAATC CACACTTGCTGAAAAAAACAATTTGCAAGGCTTATCACGTGAAGCGTTTATCTGTGAATC

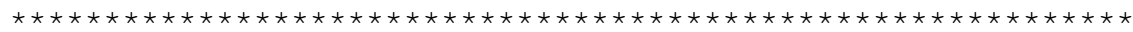

AGTGGAAATTTTATCTCTTTTAATCATACCCACCCTTTCATAGAAGGCAATGAGCGCAC AGTGGAAATTTTTATCTCTTTTAATCATACCCACCCTTTCATAGAAGGCAATGAGCGCAC

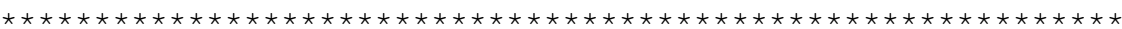

CCAACGGTTGTTTTTTCAACAATTTGCGCAAATAGCGGGCCATCAGCTGGATTTTTTACT CCAACGGTTGTTTTTTCAACAATTTGCGCAAATAGCGGGCCATCAGCTGGATTTTTTACT

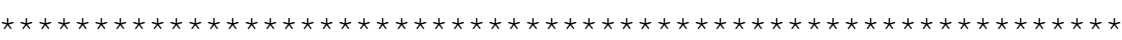

CGTAACAAAAGAACGCATGCTAGTTGCCAGTCTTGCAGCTGCACAAGATAGTCAATCTAG CGTAACAAAAGAACGCATGCTAGTTGCCAGTCTTGCAGCTGCACAAGATAGTCAATCTAG

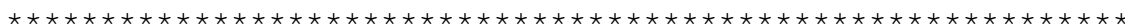


pET28a+BqBepA1_insert

BqBepA1_1

pET28a+BqBepA1_insert

BqBepA1_1

pET28a+BqBepA1_insert BqBepA1_1
AGCCTATGAAACATCTGTTTGACTCGAGCACCACCACCACCACCACTGAGATCCGGCTGC AGCCTATGAAACATCTGTTTGACTCGAGCACCACCACCACCACCACTGAGATCCGGCTGC

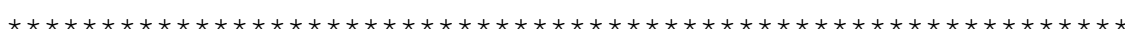

TAACAAAGCCCGAAAGGAAGCTGAGTTGGCTGCTGCCACCGCTGAGCAATAACTAGCATA TAACAAAGCCCGAAAGGAAGCTGAGTTGGCTGCTGCCACCGCTGAGCAATAACTAGCATA

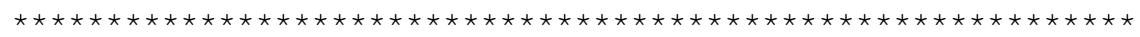

ACCCCTTGGGGCCTCTAAACGGGTCTTGAGGGGTTTTTT ACCCCTTGGGGCCTCTAAACGGGTCTTGAGGGGTTTTTT

Appendix Figure 9.4: BqBepA1 sequence alignment. pET28a sequence data was obtained from Invitrogen, BepA1 sequence data was obtained from NCBI for B. quintana JK-31 strain. pET28a insert sequence was generated by inserting the target BepA1 sequence between the $\mathrm{Ndel}$ and $\mathrm{Xhol}$ cut sites. 


\subsubsection{B. quintana BepC Alignment}

pET28a+BqBepCFic_insert TAATACGACTCACTATAGGGGAATTGTGAGCGGATAACAATTCCCCTCTAGAAATAATTT BqBepCFic_2 TAATACGACTCACTATAGGGGAATTGTGAGCGGATAACAATTCCCCTCTAGAAATAATTT

pET28a+BqBepCFic_insert TGTTTAACTTTAAGAAGGAGATATACCATGGGCAGCAGCCATCATCATCATCATCACAGC BqBepCFic_2 TGTTTAACTTTAAGAAGGAGATATACCATGGGCAGCAGCCATCATCATCATCATCACAGC pET28a+BqBepCFic_insert BqBepCFic_2

AGCGGCCTGGTGCCGCGCGGCAGCCATATGGCTAGCATGTTAGAGCATAATTATTTTTAT AGCGGCCTGGTGCCGCGCGGCAGCCATATGGCTAGCATGTTAGAGCATAATTATTTTTAT

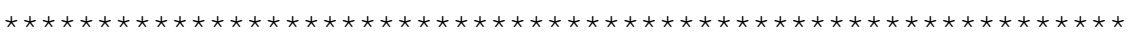
pET28a+BqBepCFic_insert BqBepCFic_2

AAAAACAGCGCAACACTGAAGAATAAACATGGCATAAAAAACCCGCGAAAACTGTATGAA AAAAACAGCGCAACACTGAAGAATAAACATGGCATAAAAAACCCGCGAAAACTGTATGAA

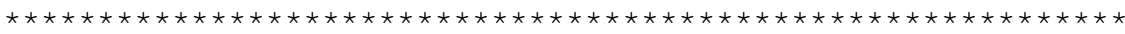

pET28a+BqBepCFic_insert BqBepCFic_2

CGCTGTGCTCATGAGACAGCCAGAGAGGCTGTAAATTTTCGCCTTGAACCGCCACCAGGG CGCTGTGCTCATGAGACAGCCAGAGAGGCTGTAAATTTTCGCCTTGAACCGCCACCAGGG

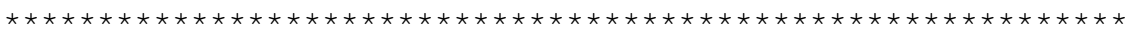

pET28a+BqBepCFic_insert BqBepCFic_2

AAATTTGATGCCGCTTATCTAAGGACAATTCACTGGTGCCTTTTCCATAAAACTTTTGAA AAATTTGATGCCGCTTATCTAAGGACAATTCACTGGTGCCTTTTCCATAAAACTTTTGAA

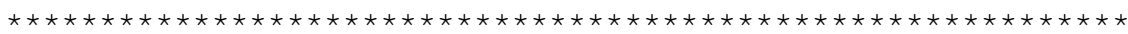

pET28a+BqBepCFic_insert BqBepCFic_2

TGGGCCGGTGTTACCCGAGATCAGCCCTTTACATTTGAAGATGGCAGCACTGCATGTATG TGGGCCGGTGTTACCCGAGATCAGCCCTTTACATTTGAAGATGGCAGCACTGCATGTATG

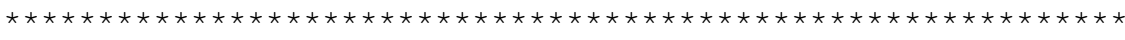

pET28a+BqBepCFic_insert BqBepCFic_2

CCAGCTATGCGACCAAAAGGTTATAAGGTTCCTTTTGCTGTCGGTTCACAAATTCAAAGA CCAGCTATGCGACCAAAAGGTTATAAGGTTCCTTTTGCTGTCGGTTCACAAATTCAAAGA

pET28a+BqBepCFic_insert BqBepCFic_2

GAGCTTAAAAAATTAGAACAAAGACTAACCGCGAAGAATAATTTACAAGGCTTATCGCGC GAGCTTAAAAAATTAGAACAAAGACTAACCGCGAAGAATAATTTACAAGGCTTATCGCGC

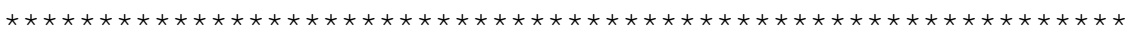

pET28a+BqBepCFic_insert BqBepCFic_2

CAAGAATTTGCTGCAAATGCTGCTGAAGTTTTTACAGCTCTCGACCACGCGCATCCTTTC CAAGAATTTGCTGCAAATGCTGCTGAAGTTTTTACAGCTCTCGACCACGCGCATCCTTTC

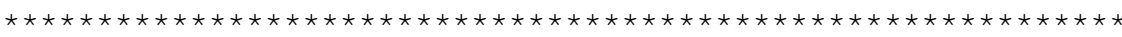

pET28a+BqBepCFic_insert BqBepCFic_2

AGAAAAGGCAATGGGCGCACACAACGAATGTTTATGGAAAAACTCGGACAAGCGGCAGGC AGAAAAGGCAATGGGCGCACACAACGAATGTTTATGGAAAAACTCGGACAAGCGGCAGGC

pET28a+BqBepCFic_insert BqBepCFic_2

TATAAGATTGATTTTCTTTGATCACAAAAGAACGCATGACATATGCCAGCATTGAAGCA TATAAGATTGATTTTCTTTGATCACAAAAGAACGCATGACATATGCCAGCATTGAAGCA

pET28a+BqBepCFic_insert

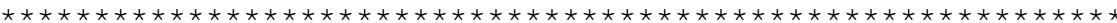
BqBepCFic_2 


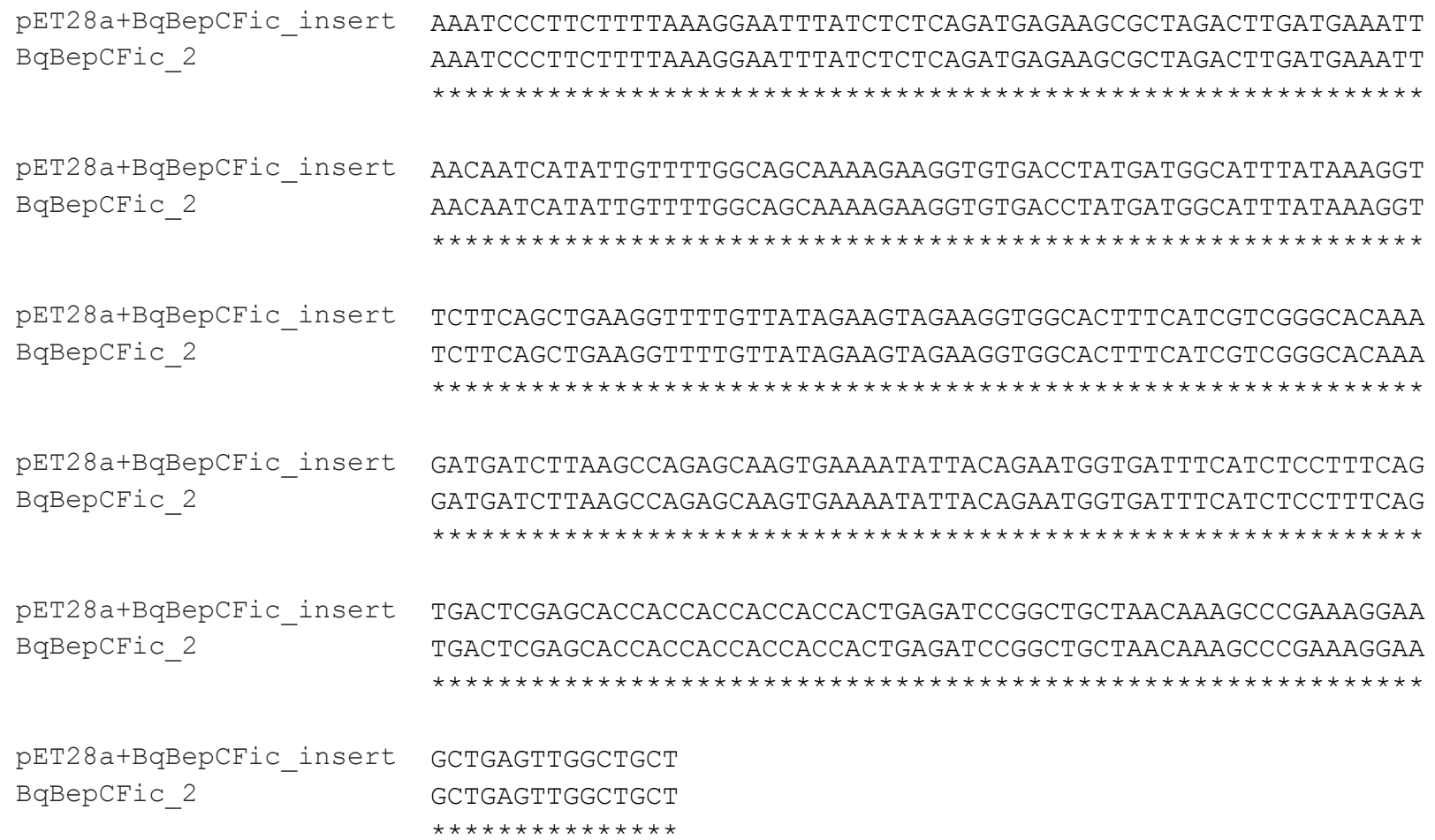

Appendix Figure 9.5: BqBepC-Fic sequence alignment. pET28a sequence data was obtained from Invitrogen, BepC sequence data was obtained from NCBI for B. quintana JK-31 strain. pET28a insert sequence was generated by inserting the target $\mathrm{BepC}$ sequence between the Nhel and Xhol cut sites, along with an additional stop codon preceding the Xhol cut site. 


\subsubsection{B. rochalimae Bep2}

pET28a+BrBep2Fic_insert TTTGTTTAACTTTAAGAAGGAGATATACCATGGGCAGCAGCCATCATCATCATCATCACA BrBep2Fic_5

pET28a+BrBep2Fic_insert BrBep2Fic 5

pET28a+BrBep2Fic insert BrBep2Fic 5

pET28a+BrBep2Fic_insert BrBep2Fic_5

pET28a+BrBep2Fic_insert BrBep2Fic_5 TTTGTTTAACTTTAAGAAGGAGATATACCATGGGCAGCAGCCATCATCATCATCATCACA

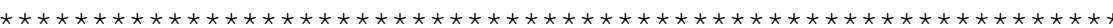

GCAGCGGCCTGGTGCCGCGCGGCAGCCATATGGCTAGCATGACTGGTGGACAGCAAATGG GCAGCGGCCTGGTGCCGCGCGGCAGCCATATGGCTAGCATGACTGGTGGACAGCAAATGG

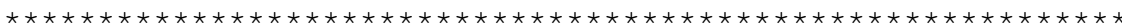

GTCGCGGATCCATGAAGAAAAGTGAAATGATGATAAAGTTGGAGGATATTAACATCCCTT GTCGCGGATCCATGAAGAAAAGTGAAATGATGATAAAGTTGGAGGATATTAACATCCCTT

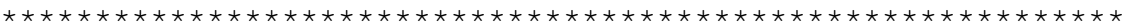

CTCCTAAAAATTATGTCTATCCTGACAGTAGAGTGTTGAAGAATAAATATGGAATCATGG CTCCTAAAAATTATGTCTATCCTGACAGTAGAGTGTTGAAGAATAAATATGGAATCATGG

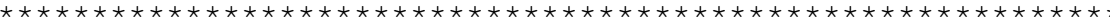

AGCTTCAAAAGTTCGAGGAGAGAATGACGCATGATGCATTAAAAGAAACTGTTAAGGTGC AGCTTCAAAAGTTCGAGGAGAGAATGACGCATGATGCATTAAAAGAAACTGTTAAGGTGC



pET28a+BrBep2Fic_insert BrBep2Fic_5

TAGAAGAACCTGCTCCAGAGCGGTTTGATTCTTCTTATCTAAAGTATTTGCATAAACGTT TAGAAGAACCTGCTCCAGAGCGGTTTGATTCTTCTTATCTAAAGTATTTGCATAAACGTT

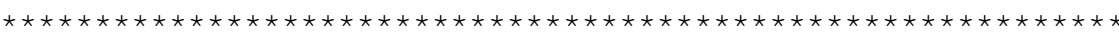

pET28a+BrBep2Fic_insert BrBep2Fic 5

TATTTCAGCATGCATTGGAATGGGCTGGGTGCACCCGTGATGTTCCATTTACATTTTTAG TATTTCAGCATGCATTGGAATGGGCTGGGTGCACCCGTGATGTTCCATTTACATTTTTAG

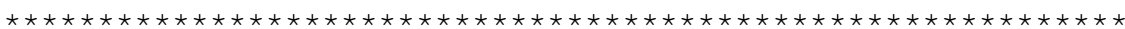

pET28a+BrBep2Fic_insert BrBep2Fic_5

pET28a+BrBep2Fic_insert BrBep2Fic_5

pET28a+BrBep2Fic_insert BrBep2Fic_5

ATGGTACAAGGGCTGTGATGCCCTCCATGGCAAAGTGTGATTCCGATTTTGGTTTTGCAT ATGGTACAAGGGCTGTGATGCCCTCCATGGCAAAGTGTGATTCCGATTTTGGTTTTGCAT

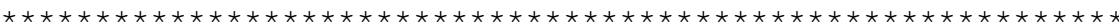

TTGGTTCAGAGATTAAGGAAGGTTTAGATTTCATCGATCAAAAACTCTCTGAGAAGAATT TTGGTTCAGAGATTAAGGAAGGTTTAGATTTCATCGATCAAAAACTCTCTGAGAAGAATT

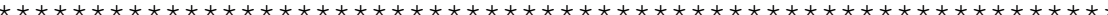

ATTTGAGAGGCTTATCGCGTGAAGAATTTGTTCATAATGCAGCAAAAATATTTGGCTCTA ATTTGAGAGGCTTATCGCGTGAAGAATTTGTTCATAATGCAGCAAAAATATTTGGCTCTA

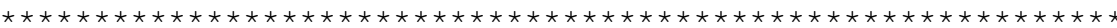

pET28a+BrBep2Fic_insert BrBep2Fic_5

TCAATTATATTCATCCTTTTAGGGAAGGTAATGGACGCGTACAAAGATTGTTTTTTGTAA TCAATTATATTCATCCTTTTAGGGAAGGTAATGGACGCGTACAAAGATTGTTTTTTGTAA

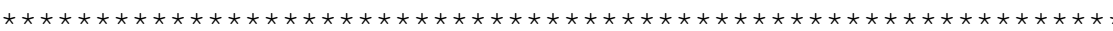

pET28a+BrBep2Fic_insert BrBep2Fic 5

AGCTAGCCAGATCTGTAGGCTATAAACTTGACTTTTCCGCTACAACAACAAAGCGCATGA AGCTAGCCAGATCTGTAGGCTATAAACTTGACTTTTCCGCTACAACAACAAAGCGCATGA

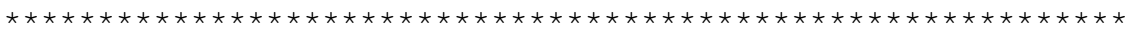

pET28a+BrBep2Fic_insert BrBep2Fic_5

CATATGTGAGTGTTGAGGTTACAAAAAACGCATATATGAAGCCTTTGCAACATTTGTTTG CATATGTGAGTGTTGAGGTTACAAAAAACGCATATATGAAGCCTTTGCAACATTTGTTTG

pET28a+BrBep2Fic_insert BrBep2Fic_5

AAGATATTTCCAATCCAGAAAAGGTCGGTATTTTAAAGCAATTCATAAGCAATGCCAAAG AAGATATTTCCAATCCAGAAAAGGTCGGTATTTTAAAGCAATTCATAAGCAATGCCAAAG

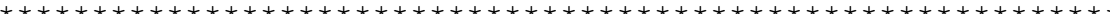




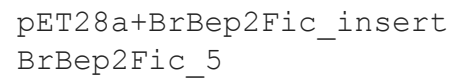

AAAAATTAGCTGCTTTGAGTGAAGATGAAATGATTGAAAAAATTTTAGAATATAGTTCTG

Appendix Figure 9.6: Bep2-Fic sequence alignment. pET28a sequence data was obtained from Invitrogen, Bep2 sequence data was obtained from NCBI based of sequence data from Pieles et al. (2014) for B. rochalimae. pET28a insert sequence was generated by inserting the target Bep2 sequence between the BamHI and Xhol cut sites, along with an additional stop codon before the Xhol cut site.. AAAAATTAGCTGCTTTGAGTGAAGATGAAATGATTGAAAAAATTTTAGAATATAGTTCTG
$* * * * * * * * * * * * * * * * * * * * * * * * * * * * * * * * * * * * * * * * * * * * * * * * * * * * * * * * * * * *$

AATATAATATCTTTGATTCGAATGAAAAGTTGATTACAGTAGCGAGAGAAGGTGAAACCT AATATAATATCTTTGATTCGAATGAAAAGTTGATTACAGTAGCGAGAGAAGGTGAAACCT

ATAAGGGGATTGGTAAATTCCTTGCTTCAGATTTTATTGTAATAGAAGCGGGAGATACTT ATAAGGGGATTGGTAAATTCCTTGCTTCAGATTTTATTGTAATAGAAGCGGGAGATACTT

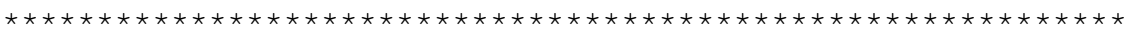

GTATCCTCTCCAATAAAGATTCCCTTACACCAGAACAATTAAGGACATTCAAACTTGGTA GTATCCTCTCCAATAAAGATTCCCTTACACCAGAACAATTAAGGACATTCAAACTTGGTA

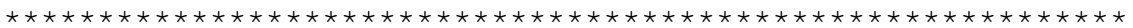

GTGATTTCATTTTTACAGCTTTAGATACTAAAGATCTTAAAGAAATTTTTATCCCAGAAG GTGATTTCATTTTTACAGCTTTAGATACTAAAGATCTTAAAGAAATTTTATCCCAGAAG

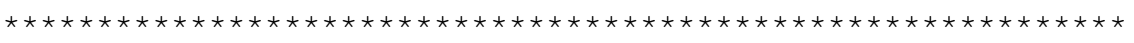




\subsubsection{Vibrio parahaemolyticus VopS}

pET28a+VpVopS_10

VpVops_10

pET28a+VpVops_10

VpVops_10

pET28a+VpVopS_10 VpVops_10

pET28a+VpVopS_10 VpVops_10

pET28a+VpVopS_10 VpVops_10

pET28a+VpVopS_10 VpVops_10

pET28a+VpVopS_10 VpVops_10

pET28a+VpVops_10 VpVops_10

pET28a+VpVops_10 VpVops_10

pET28a+VpVopS_10 VpVops_10

pET28a+VpVopS_10 VpVops_10

pET28a+VpVopS_10 VpVops_10

pET28a+VpVops_10 VpVops_10
TTTGTTTAACTTTAAGAAGGAGATATACCATGGGCAGCAGCCATCATCATCATCATCACA TTTGTTTAACTTTAAGAAGGAGATATACCATGGGCAGCAGCCATCATCATCATCATCACA

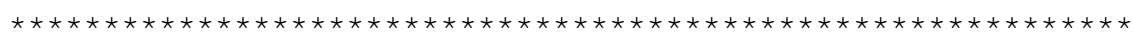

GCAGCGGCCTGGTGCCGCGCGGCAGCCATATGATCAGTTTTGGAAGTGTGAGTGCATTGC GCAGCGGCCTGGTGCCGCGCGGCAGCCATATGATCAGTTTTGGAAGTGTGAGTGCATTGC

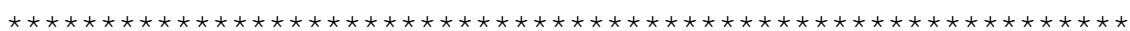

AAGCGGCGATGCCACAAGCTCGTAATGAGATTCTAAATGAAGGCAAACTCAGCATTGGTG AAGCGGCGATGCCACAAGCTCGTAATGAGATTCTAAATGAAGGCAAACTCAGCATTGGTG

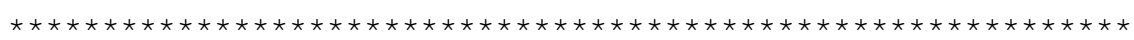

GTAAGGAATATACGATCAATGCTGCTACTCAAGAGTTTACACGCGCAAACCCTACAAGTG GTAAGGAATATACGATCAATGCTGCTACTCAAGAGTTTACACGCGCAAACCCTACAAGTG

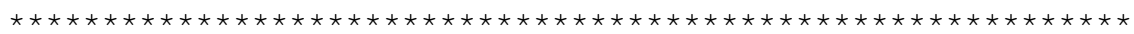

GTGCAGTCGCGCGCTTCTTTGAAGCTACTGGGAAATTATTTCGTGAAGGCAGCACGCAGT GTGCAGTCGCGCGCTTCTTTGAAGCTACTGGGAAATTATTTCGTGAAGGCAGCACGCAGT

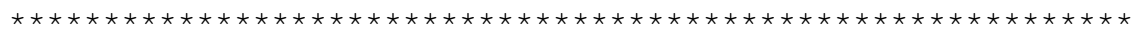

CTGTGGCAAAAGCGATCACAAAAGCAGTGTTTGACAACGAACAGGGACAAGCTCAGCGTT CTGTGGCAAAAGCGATCACAAAAGCAGTGTTTGACAACGAACAGGGACAAGCTCAGCGTT

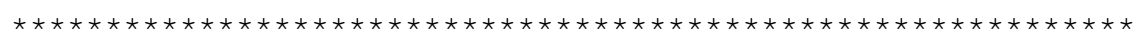

TGCAAACATCGTCTTCTGTCGAGCATGGGCAGATGTTGTTTAAAGACGCGAACCTCAAAA TGCAAACATCGTCTTCTGTCGAGCATGGGCAGATGTTGTTTAAAGACGCGAACCTCAAAA

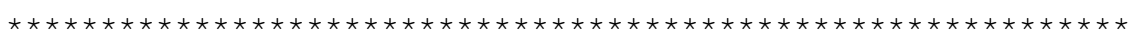

CGCCTTCAGATGTTCTGAATGCGTTTGCGAAGTTGGACAGCAAAATGGTGAAGTCTCACG CGCCTTCAGATGTTCTGAATGCGTTTGCGAAGTTGGACAGCAAAATGGTGAAGTCTCACG

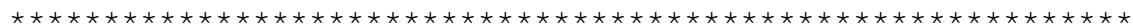

CTGCTGAGCTGAGCCAACTTGCCGAACGAGCCATGACAGAAGTGATGCTAGAGACGGATT CTGCTGAGCTGAGCCAACTTGCCGAACGAGCCATGACAGAAGTGATGCTAGAGACGGATT

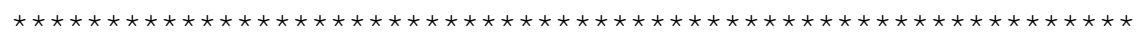

CTGGCAAGAACCTCAAAGCGCTGATTGGTGATGATGCAGTTAAATCGCTCGCGGTGCGTG CTGGCAAGAACCTCAAAGCGCTGATTGGTGATGATGCAGTTAAATCGCTCGCGGTGCGTG

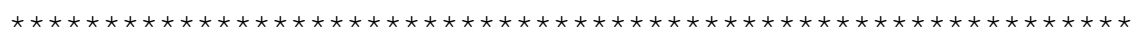

TGGTGAAAGATTACGGCGGTGGCGTGGCGGCTGCTCAGAAAAATCCAGAAGTACGCATCA TGGTGAAAGATTACGGCGGTGGCGTGGCGGCTGCTCAGAAAAATCCAGAAGTACGCATCA

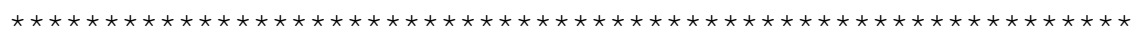

ACCAAATGCAAGCTGTCTTTGACATGGAGGTGATGCACCTGAAAGCAGCGCAGCGACATA ACCAAATGCAAGCTGTCTTTGACATGGAGGTGATGCACCTGAAAGCAGCGCAGCGACATA

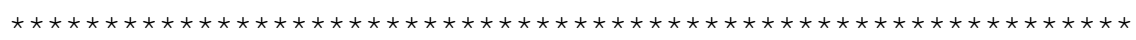

TTGAAGGGTTAGCAAGTACGGATTTAGACCAAGGCGTGTATGCAGAAGGTCTTCCTGAAG TTGAAGGGTTAGCAAGTACGGATTTAGACCAAGGCGTGTATGCAGAAGGTCTTCCTGAAG

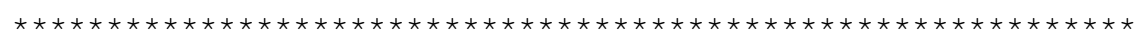




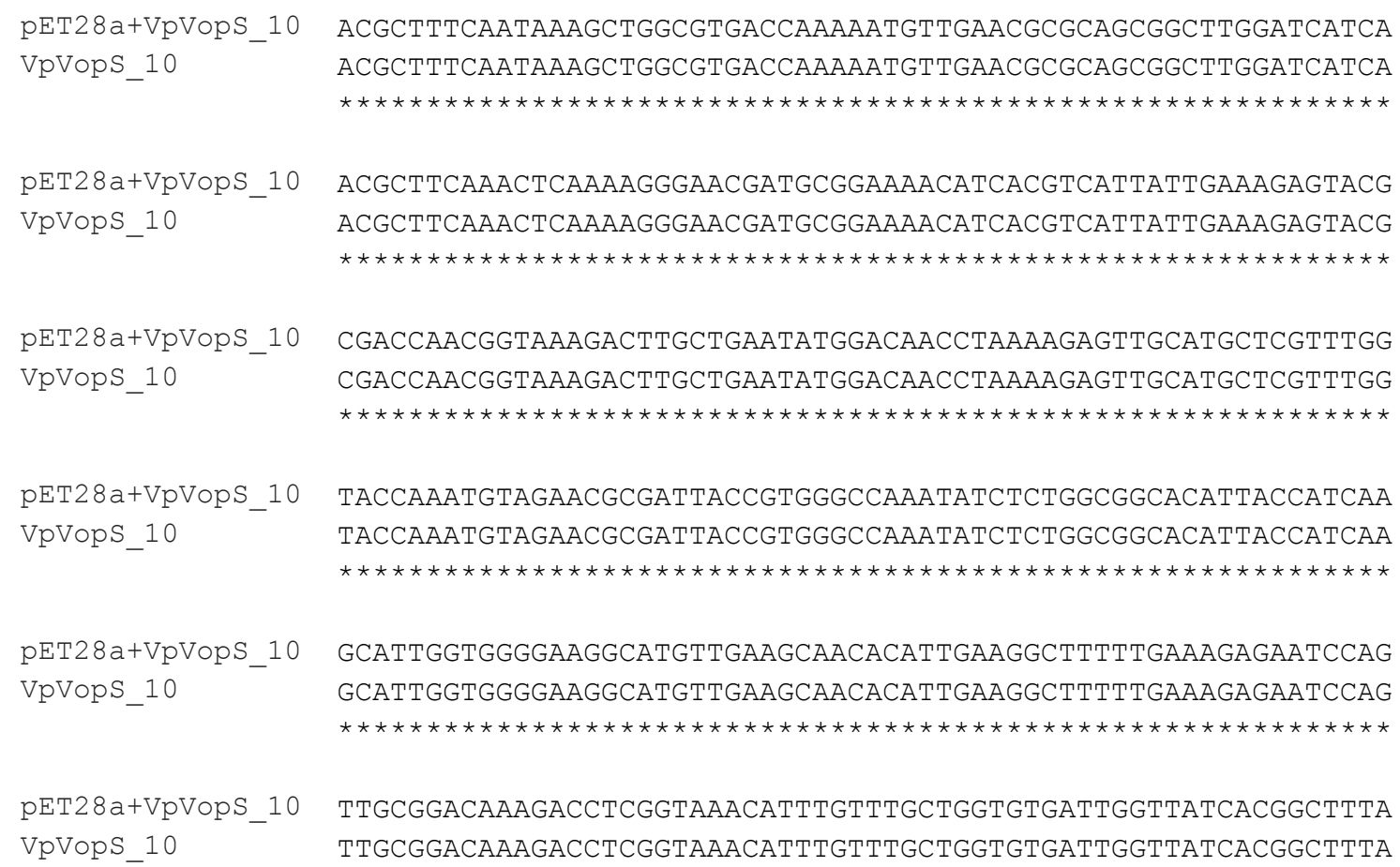

Appendix Figure 9.7: VopS sequence alignment. pET28a sequence data was obtained from Invitrogen, VopS sequence data was obtained from NCBI for Vibrio parahaemolyticus serotype O4:K12.. pET28a insert sequence was generated by inserting the target VopS sequence between the Ndel and Xhol cut sites. 


\subsection{Protein Induction Gels}

\subsubsection{B. henselae BhBepA-Fic}

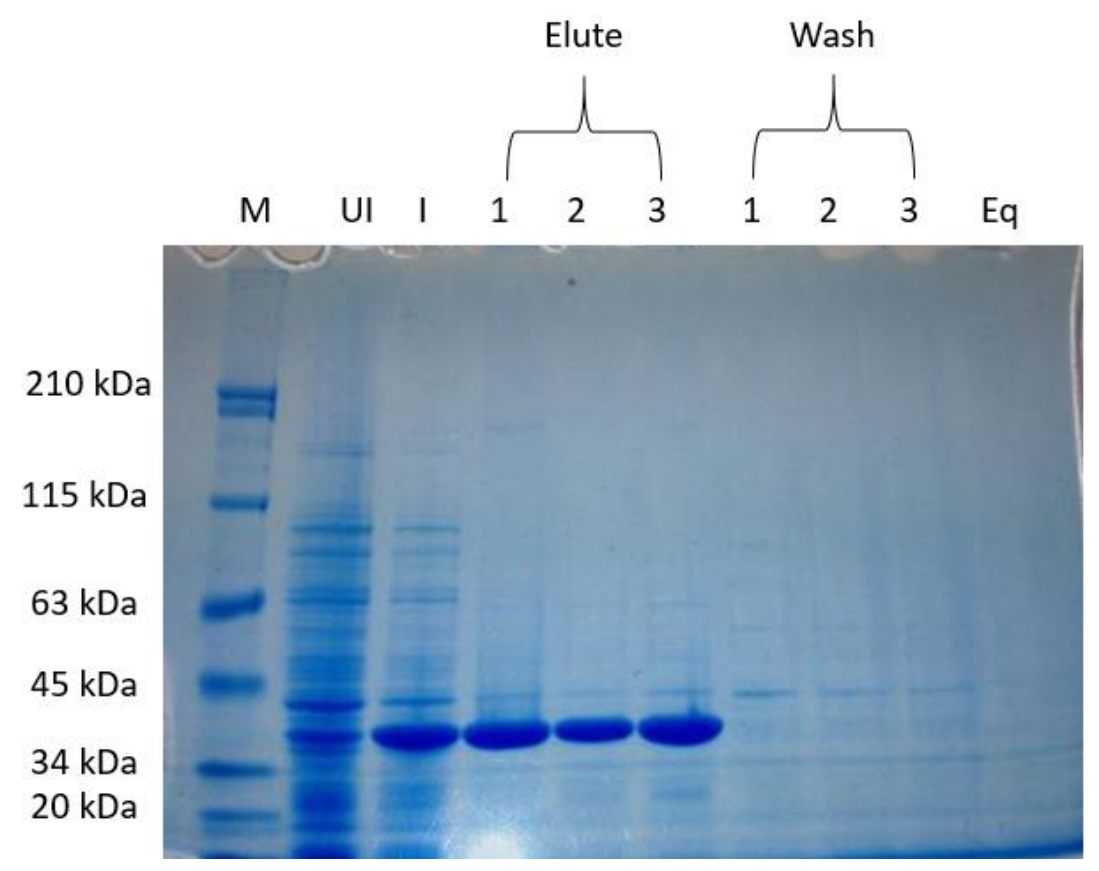

Appendix Figure 9.8: Nickel column affinity purification of BhBepA-Fic protein. The dark bands in the elution samples correspond with the predicted weight for the BhBepA-Fic protein. $\mathrm{M}=$ Marker, $\mathrm{UI}=$ Uninduced protein, $\mathrm{I}=$ Induced total protein, $\mathrm{Eq}=$ Equilibration sample.

\subsubsection{B. henselae BhBepA_H159A-Fic}

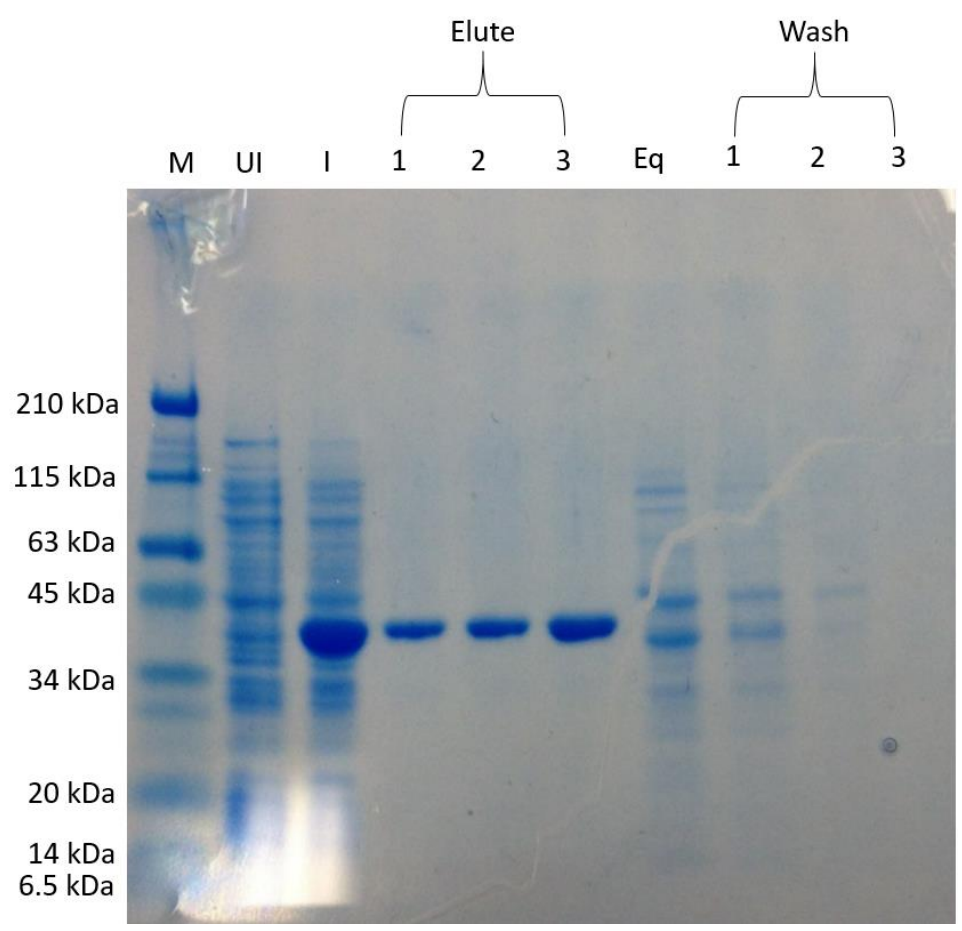

Appendix Figure 9.9: Nickel column affinity purification of BhBepA_H159A-Fic. The dark bands in the elution samples correspond with the predicted weight for the BhBepA_H159A-Fic protein. $\mathrm{M}=$ Marker, $\mathrm{UI}=$ Uninduced protein, I = Induced protein, $\mathrm{Eq}=$ Equilibration sample. 


\subsubsection{B. henselae BhBepB-Fic}

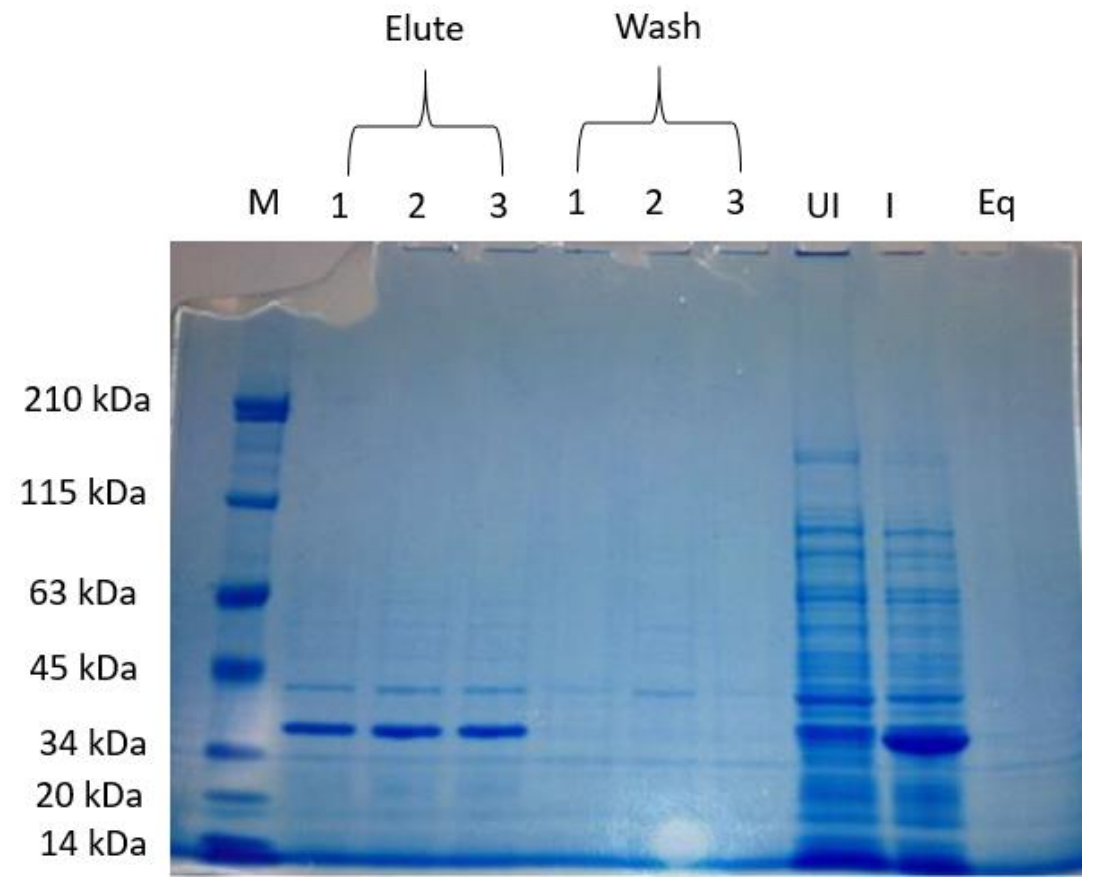

Appendix Figure 9.10: Nickel affinity purification of BhBepB-Fic protein. The dark bands in the elution samples correspond with the predicted weight for the BhBepB-Fic protein. $\mathrm{M}=$ Marker, $\mathrm{UI}=$ Uninduced protein, $\mathrm{I}=\mathrm{Induced}$ protein, Eq = Equilibration sample.

\subsubsection{B. henselae BhBepC-Fic}

(a)

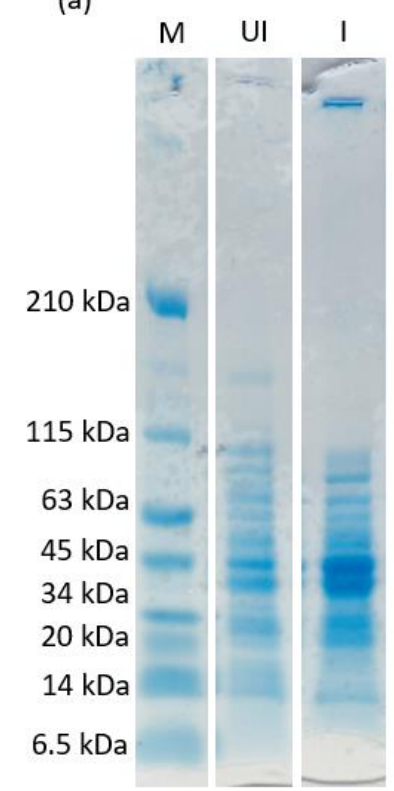

(b)

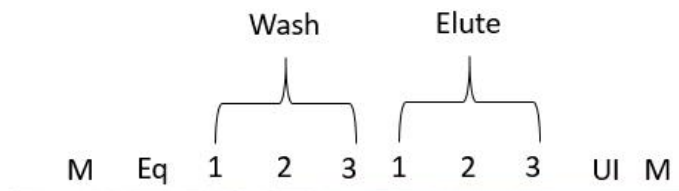

$210 \mathrm{kDa}$

$115 \mathrm{kDa}$

$63 \mathrm{kDa}$

$45 \mathrm{kDa}$

$34 \mathrm{kDa}$

$20 \mathrm{kDa}$

$14 \mathrm{kDa}$

$6.5 \mathrm{kDa}$

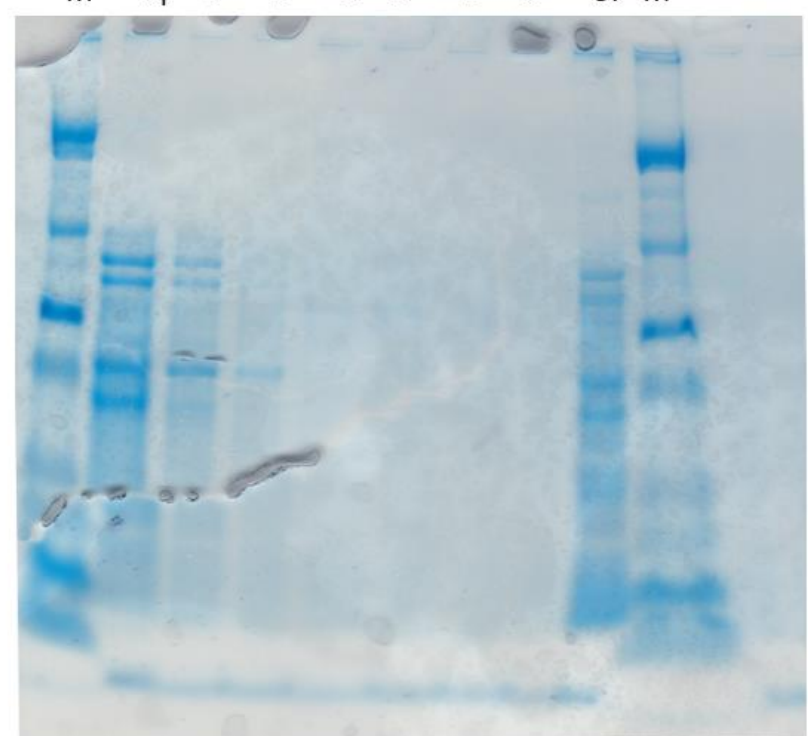

Appendix Figure 9.11: Nickel affinity purification of BhBepC-Fic protein. (a) Induction samples with dark band in the Induced sample indicating a successful BhBepC-Fic induction. (b) Purification samples of BhBepC-Fic. The lack of bands in the elution samples corresponding to the molecular weight of BhBepC-Fic, suggests the protein is insoluble $\mathrm{M}=\mathrm{Marker}, \mathrm{UI}=\mathrm{Uninduced}$ protein, I = Induced protein, $\mathrm{Eq}=$ Equilibration sample. 


\subsubsection{B. quintana BqBepA1}

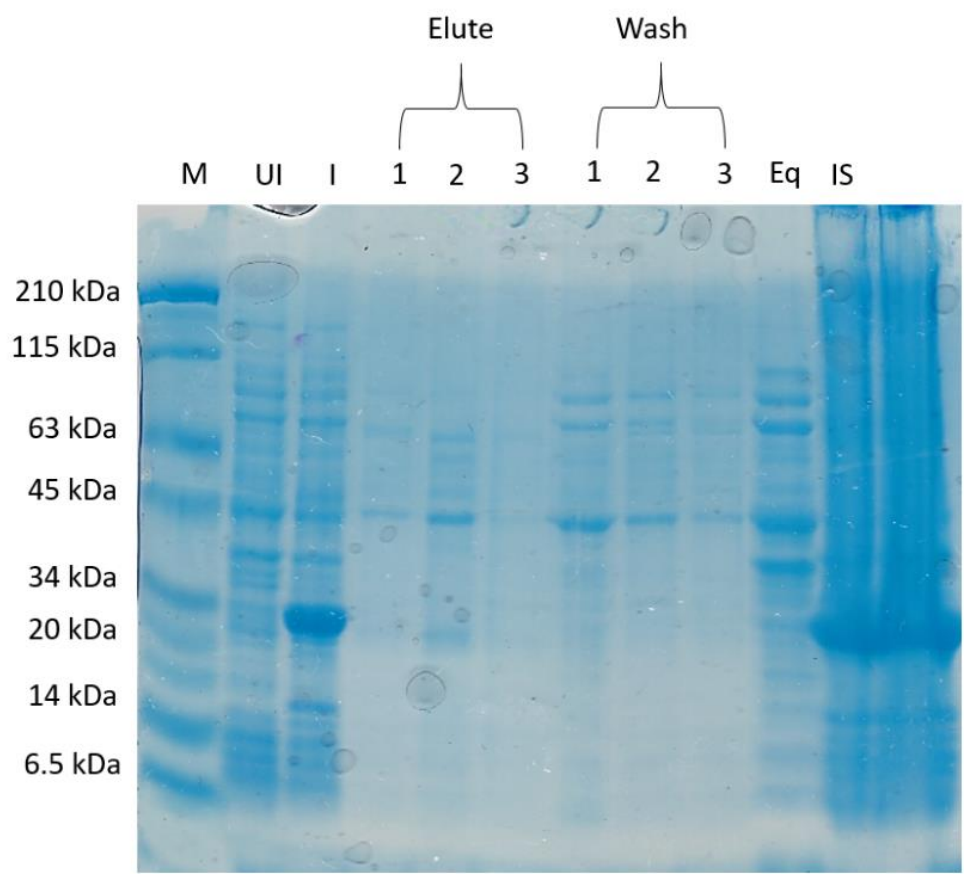

Appendix Figure 9.12: Nickel affinity purification of BqBepA1 protein. The dark bands in the induced and insoluble samples correspond with the predicted weight for the BqBepA1 protein. While there is clear evidence of protein induction, it was not purified in the elution samples and remained in the insoluble samples. Insoluble samples were redissolved in $8 \mathrm{M} U$ rea. $\mathrm{M}$ $=$ Marker, $\mathrm{UI}=$ Uninduced protein, I = Induced protein, Eq = Equilibration sample, IS = Insoluble protein .

\subsubsection{B. quintana BqBepC-Fic}



Appendix Figure 9.13: Nickel affinity purification of BqBepC-Fic protein. The dark bands in the induced and insoluble samples correspond with the predicted weight for the BqBepC-Fic protein. While there is clear evidence of protein induction, it was not purified in the elution samples and remained in the insoluble samples. Insoluble samples were redissolved in $8 \mathrm{M}$ Urea. $\mathrm{M}=$ Marker, $\mathrm{UI}=$ Uninduced protein, $\mathrm{I}=$ Induced protein, $\mathrm{Eq}=$ Equilibration sample, $\mathrm{IS}=$ Insoluble protein. 


\subsubsection{B. rochalimae Bep2-Fic}

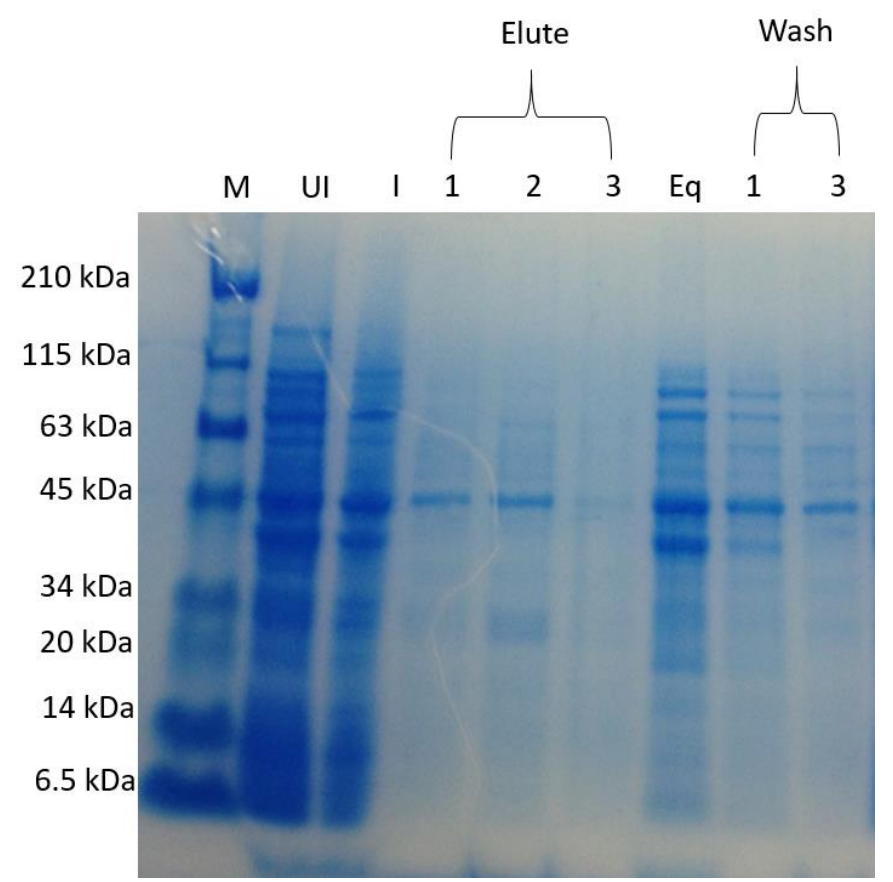

Appendix Figure 9.14: Nickel affinity purification of BrBep2-Fic protein. The bands in the elution samples correspond with the predicted weight for the BrBep2-Fic protein. $\mathrm{M}=$ Marker, $\mathrm{UI}=$ Uninduced protein, $\mathrm{I}=\mathrm{Induced}$ protein, Eq = Equilibration sample.

\subsubsection{Vibrio parahaemolyticus VopS}

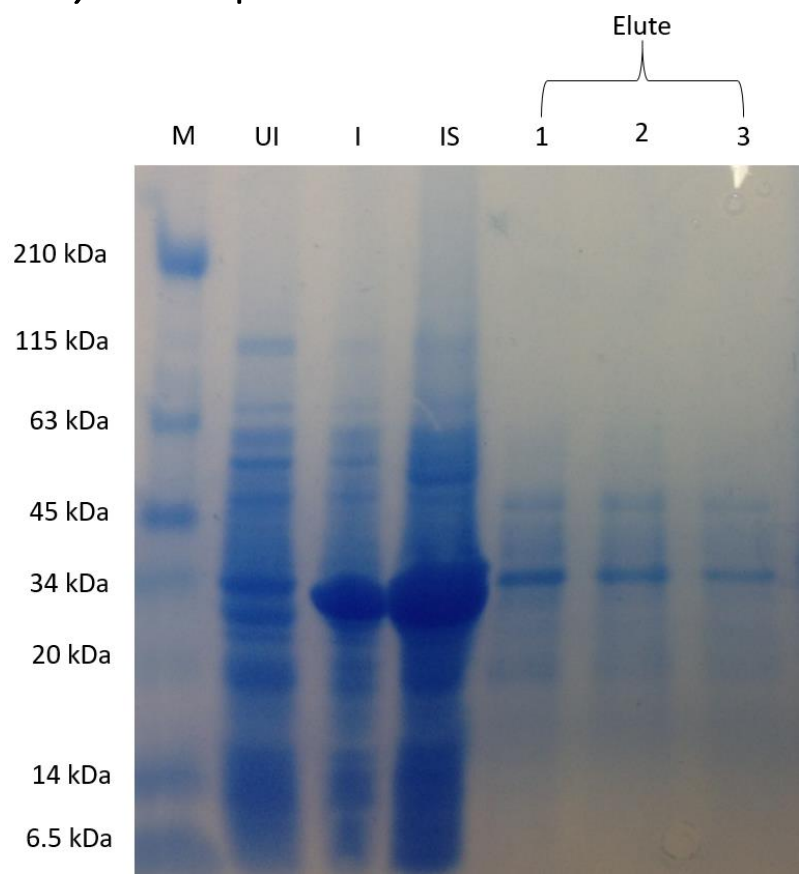

Appendix Figure 9.15: Nickel affinity purification of VopS protein. The bands in the elution samples correspond with the predicted weight for the VopS, although there is a large number of protein still in the Insoluble fraction. $\mathrm{M}=\mathrm{Marker}, \mathrm{UI}=$ Uninduced protein, I = Induced protein, IS = insoluble protein. 


\subsection{Pyrophosphate Analysis}

\section{Pyrophosphate analysis of Bep-Fic treated vimentin samples}

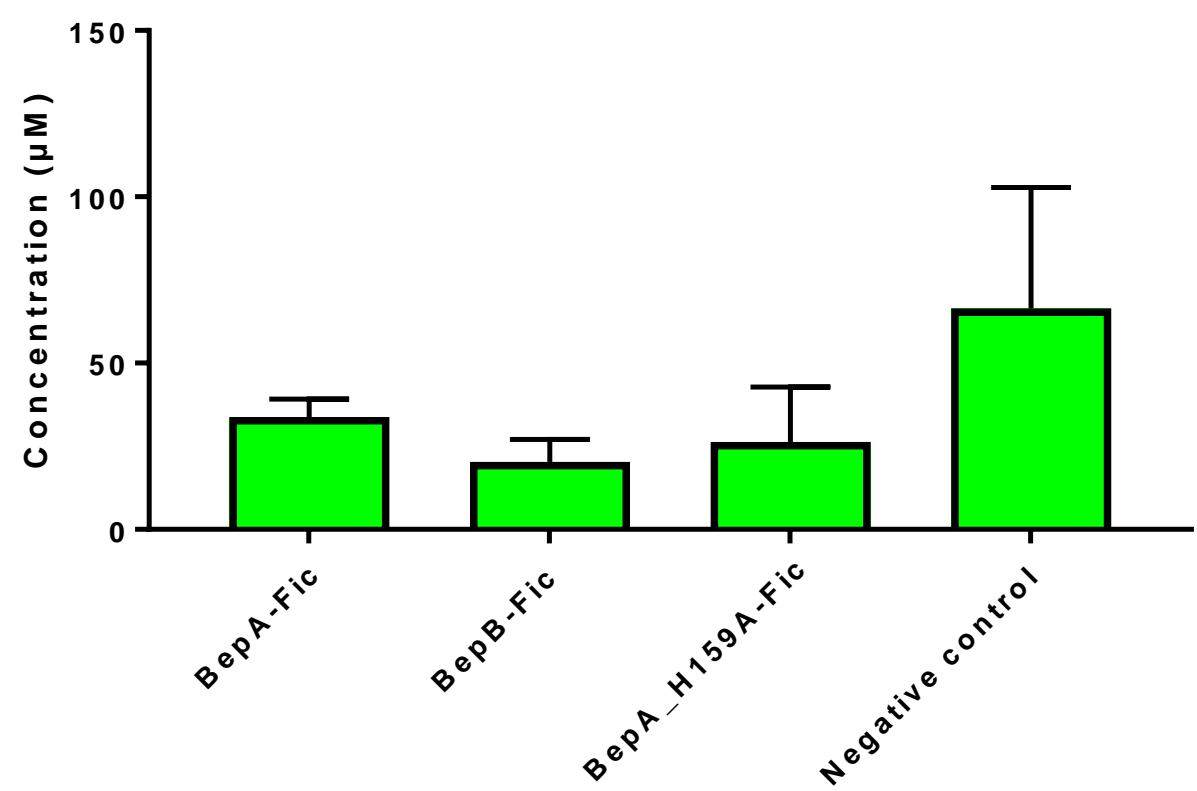

Appendix Figure 9.16: Pyrophosphate reactions for $B$. henselae Fic proteins on vimentin samples. The graph indicates no significant difference in the levels of pyrophosphate between samples and controls, suggesting no detectable change in pyrophosphate occurred during the reaction. Standard curve $R^{2}=0.9608$.

\section{Pyrophosphate analysis of Bep-Fic treated whole cell extract samples}

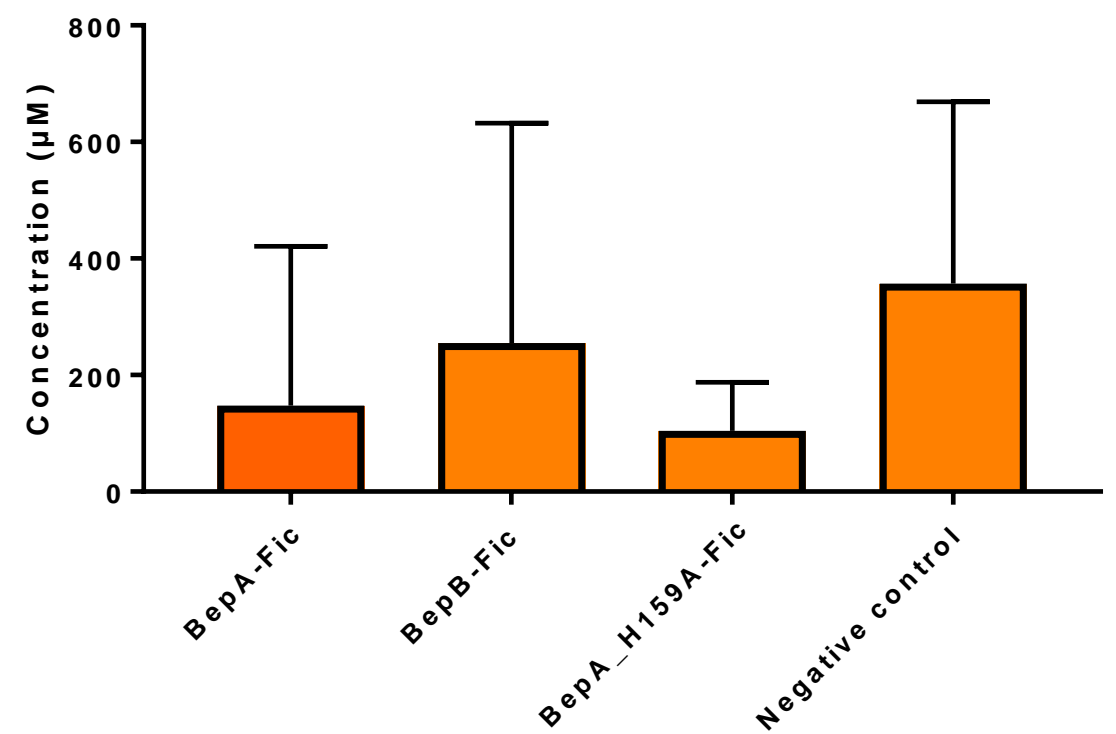

Appendix Figure 9.17: Pyrophosphate reactions for B. henselae Fic proteins on WCE samples. The graph indicates no significant difference in the levels of pyrophosphate between samples and controls. Data obtained was significantly more varied than initial vimentin experiments above, indicating the freeze-thawing of the pyrophosphate reagent may have decreased accuracy. Standard curve $\mathrm{R}^{2}=0.796$. 